UNIVERSIDADE DE SÃO PAULO

ESCOLA DE ENGENHARIA DE SÃO CARLOS

DEPARTAMENTO DE ENGENHARIA ELÉTRICA

JORGE ANDREY DA SILVA MACÊDO

\title{
Formalismo FDTD para a modelagem de meios dispersivos apresentando anisotropia biaxial
}

Dissertação apresentada à Escola de

Engenharia de São Carlos da

Universidade de São Paulo como parte dos requisitos para obtenção do Título de Mestre em Engenharia Elétrica.

Área de concentração: Telecomunicações

Orientador: Prof. Dr. Ben-Hur Viana Borges

São Carlos 2008 
Aos meus familiares, amigos e professores. 


\section{AGRADECIMENTOS}

Ao Conselho Nacional de Desenvolvimento Científico e Tecnológico, pelo suporte a este trabalho.

Aos professores e alunos do Departamento de Engenharia Elétrica da Escola de Engenharia de São Carlos - EESC/USP. 
"Daquilo que sabes conhecer e medir, é preciso que te despeças, pelo menos por um tempo. Somente depois de teres deixado a cidade verás a que altura suas torres se elevam acima das casas."

Friedrich Nietzsche 


\section{SUMÁRIO}

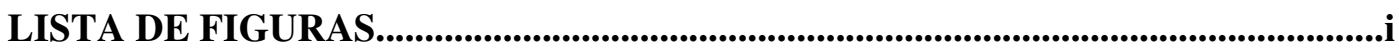

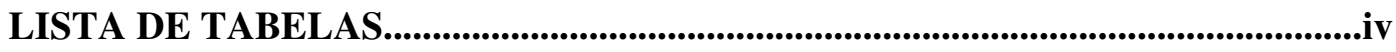

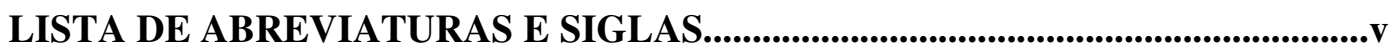

LISTA DE SÍMBOLOS................................................................................................vi

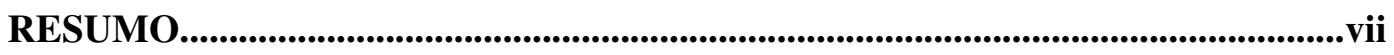

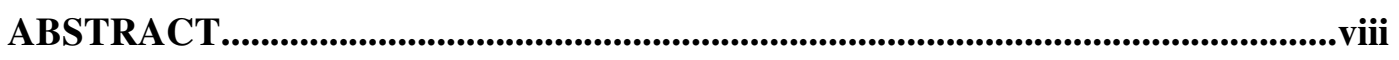

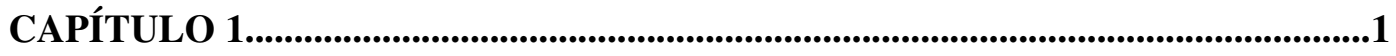

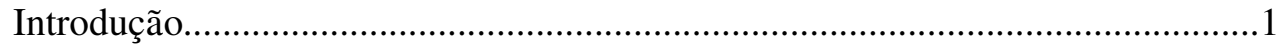

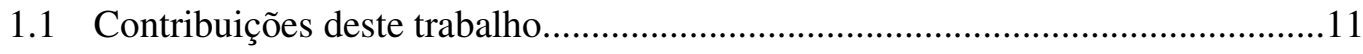

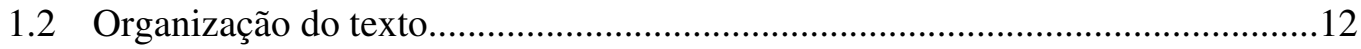

CAPÍTULO 2 FORMALISMO PARA EQUAÇÕES DE DIFERENÇAS FINITAS

NO DOMÍNIO DO TEMPO.............................................................................................13

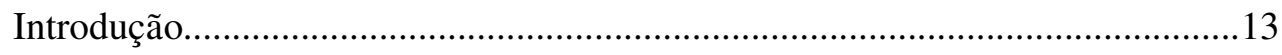

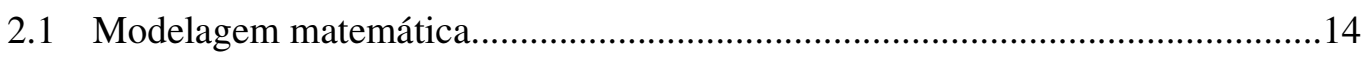

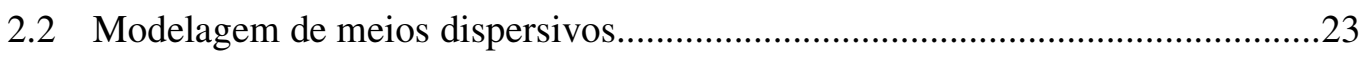

2.3 Método das diferenças finitas no domínio do tempo (FDTD)............................29

CAPÍTULO 3 RESULTADOS NUMÉRICOS..............................................................36

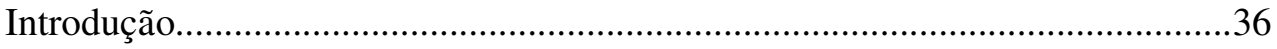

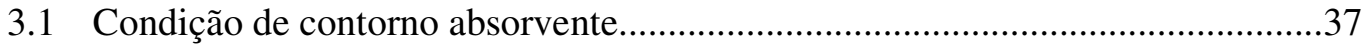

3.2 Estruturas capazes de proporcionar a manipulação da radiação

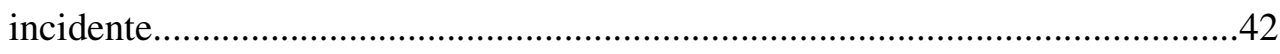

3.2.1 Cobertura de invisibilidade eletromagnética...........................................43

3.2.2 Rotacionador de campo eletromagnético................................................49

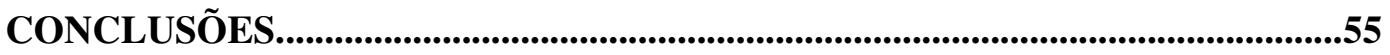

REFERÊNCIAS BIBLIOGRÁFICAS....................................................................57

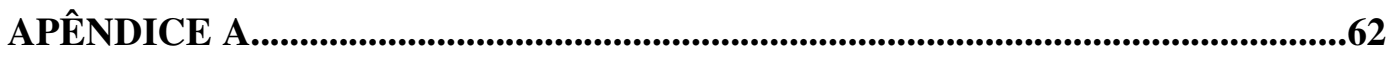

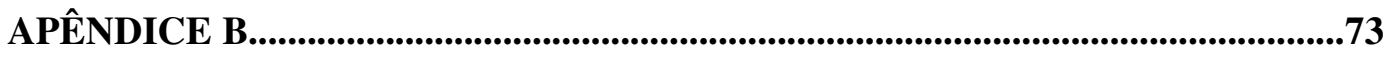

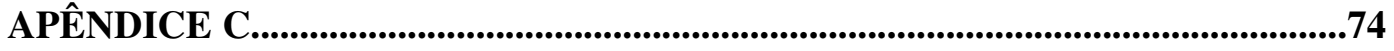




\section{LISTA DE FIGURAS}

Fig.1.1 - Arranjo tridimensional de fios condutores para produzir materiais com $\varepsilon$ negativo [4] .2

Fig.1.2 - Ressoador em anel segmentado (SRR) utilizado para obtenção de materiais $\operatorname{com} \mu$ negativo [6]...... 2

Fig.1.3 - Representação esquemática do metamaterial proposto em [3].... 3

Fig.1.4 - Estrutura desenvolvida por Shelby et al. em [7] para obter um meio isotrópico com refração negativa. .3

Fig.1.5 - Números de artigos publicados sobre metamateriais nos últimos anos, obtidos do ISI [9]. . .4

Fig.1.6 - Classificação dos materiais combinando seus parâmetros

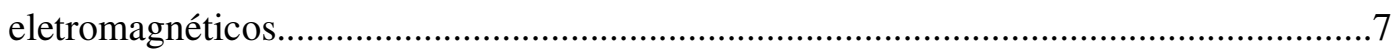

Fig.1.7 - Combinação de células 2D de linhas RHM (flecha) e LHM [15].....................

Fig.1.8 - Absorvedor baseado em metamateriais, desenvolvido em [17]...................... Fig.1.9 - Cobertura de invisibilidade eletromagnética construída com

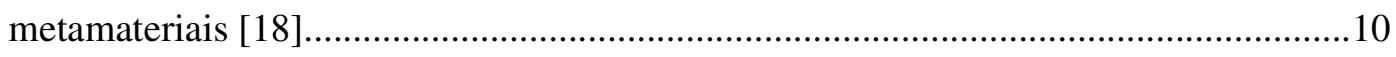

Fig.2.1 - Domínio computacional analisado neste trabalho........................................14

Fig.2.2 - Esquema ilustrativo, no plano cartesiano, da interação de um objeto com a radiação incidente gerada por uma fonte de ondas planas........................................... 16

Fig.2.3 - Esquema ilustrativo da criação de uma região nula à radiação eletromagnética, obtida a partir da expansão da origem do sistema cartesiano. A linha destacada, apenas indica o efeito da deformação do meio na trajetória de um feixe 
Fig.2.4 - Representação no sistema cartesiano. (a) antes da transformação e (b) após a transformação

Fig.2.5 - Representação do sistema de coordenadas cilíndrica no sistema cartesiano.

Fig.2.6 - Permissividade elétrica relativa modelada por Drude. Parte real (esquerda) e parte imaginária (direita), com $\Gamma_{e}=30 \mathrm{MHz}$

Fig.2.7 - Esquema leapfrog de evolução temporal do método FDTD. 30

Fig.2.8 - Célula de discretização espacial do método 2D-FDTD. 30

Fig. 3.1 - Distribuição espacial do campo elétrico gerado por uma fonte pontual situada no centro do domínio computacional, observada após 200 iterações temporais. (a) Distribuição de campo na ausência de PML e (b) Distribuição de campo utilizando a PML. .38

Fig. 3.2 - Domínio computacional para o cálculo da eficiência da PML. (a) Janela computacional suficientemente grande para obtenção do campo de referência e (b) janela computacional reduzida, envolvida pela PML. O ponto A é o local onde são tomados os campos incidente e refletido.

Fig. 3.3 - Comparação de eficiência de PML utilizada neste trabalho com a de [37] para diferentes valores de $m$, (a) $m=2$, (b) $m=3$ e (c) $m=4$

Fig. 3.4 - Distribuição espacial de $E_{z}$, (a) não considerando a dispersão dos materiais, (b) modelo de Drude incluso no formalismo do método FDTD, (c) idem (b) com passo espacial reduzido à metade do valor adotado em (a) e (b) e (d) idem (c), porém considerando na simulação a perda do material utilizado para fabricação da primeira cobertura de invisibilidade eletromagnética......

Fig. 3.5 - Distribuição espacial da componente $\mu_{x}$

Fig. 3.6 - Distribuição espacial de $E_{z}$ : (a) simulado, (b) experimental. Resultados extraídos de [18] 
Fig. 3.7 - Distribuição espacial da componente $H_{z}$ (a)após penetrar no cilindro de metamaterial, (b) completa simulação com freqüência da fonte de excitação igual a

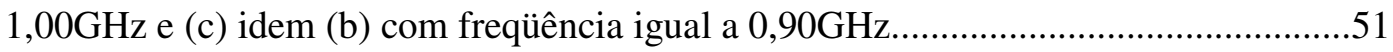

Fig. 3.8 - Distribuição espacial de $H_{z}$, (a) frequiência da fonte de excitação de $1,00 \mathrm{GHz}$,

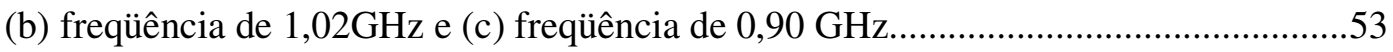

Fig. 3.9 - Espalhamento da seção reta normalizada (SCS) vs freqüência para o

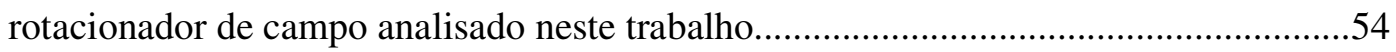




\section{LISTA DE TABELAS}

TABELA I: Parâmetros do domínio computacional empregados no teste de desempenho da PML. $N_{x}$ e $N_{y}$ representam o número de pontos do domínio computacional, $\Delta x$ e $\Delta y$ representam o passo espacial adotado na direção $x$ e $y$, respectivamente. $\Delta t$ é o passo temporal adotado, $\sigma_{\max }$ é a máxima condutividade elétrica utilizada na PML, $d$ é a espessura da PML utilizada e $m$ é o grau do polinômio da PML adotado nessa simulação. .36

TABELA II: Parâmetros do domínio computacional empregados no teste de desempenho da PML. $N_{x}$ e $N_{y}$ representam o número de pontos do domínio computacional, $\Delta x$ e $\Delta y$ representam o passo espacial adotado na direção $x$ e $y$, respectivamente. $\Delta t$ é o passo temporal adotado, $\sigma_{\max }$ é a máxima condutividade elétrica utilizada na PML, $m$ é o grau do polinômio que descreve o perfil de condutividade da PML, $d$ é a espessura da PML utilizada e $m$ é o grau do polinômio da PML adotado na simulação. 39

TABELA III: Parâmetros do domínio computacional adotados nas quatro simulações distintas da cobertura de invisibilidade eletromagnética (Fig. 3.4): (a) não considerando a dispersão dos materiais, (b) formalismo FDTD considerando o modelo de Drude, (c) considerando Drude e uma redução pela metade dos passos espaciais utilizados em (a) e (b) e (d) idem (c), entretanto considerando o fator de amortecimento do metamaterial utilizado em [18]. .43

TABELA IV: Parâmetros do domínio computacional adotados na simulação do rotacionador de campo eletromagnético. $N_{x}$ e $N_{y}$ representam o número de pontos do domínio computacional, $\Delta x$ e $\Delta y$ representam o passo espacial adotado na direção $x$ e $y$, respectivamente. $\Delta t$ é o passo temporal adotado, $\sigma_{\max }$ é a máxima condutividade elétrica utilizada na PML, $d$ é a espessura da PML utilizada e $m$ é o grau do polinômio da PML adotado na simulação. 


\section{LISTA DE ABREVIATURAS E SIGLAS}

$2 \mathrm{D}$

$\mathrm{ABC}$

ADE

DNG

DPS

ENG

FDTD

FEM

LHM

MNG

MoM

NIM

NRI - TL

PML

RHM

SRR

$\mathrm{TE}_{\mathrm{z}}$

TFSF

$\mathrm{TM}_{\mathrm{z}}$
- Bidimensional

- Absorbing Boundary Conditions

- Auxiliary Differential Equation

- Double-Negative Medium

- Double-Positive Medium

- Epsilon-Negative Medium

- Finite Difference Time Domain

- Finite Element Method

- Left-Handed Material

- Mu-Negative Medium

- Method of Moments

- Negative Index Media

- Negative Refraction Index Transmission Line

- Perfectly Matched Layer

- Right-Handed Material

- Split Ring Resonator

- Transverse-Electric mode with respect $z$

- Total Field Scattered Field

- Transverse-Magnetic mode with respect $z$ 


\section{LISTA DE SÍMBOLOS}

\begin{tabular}{|c|c|}
\hline$\vec{B}$ & - Vetor densidade de fluxo magnético (weber/metro ${ }^{2}$ ) \\
\hline$c$ & - Velocidade da luz no espaço-livre (metro/segundo) \\
\hline$\vec{D}$ & - Vetor densidade de fluxo elétrico (coulomb/metro ${ }^{2}$ ) \\
\hline$d$ & - Espessura da camada de PML (metro) \\
\hline$\vec{E}$ & - Vetor campo elétrico (volt/metro) \\
\hline$\vec{H}$ & - Vetor campo magnético (ampère/metro) \\
\hline$\vec{J}$ & - Vetor densidade de corrente elétrica (ampére/metro ${ }^{2}$ ) \\
\hline$j$ & - Unidade imaginária \\
\hline$\vec{K}$ & - Vetor densidade de corrente magnética (volts/metro ${ }^{2}$ ) \\
\hline$\vec{M}$ & - Vetor de polarização magnética (ampère/metro) \\
\hline$\vec{P}$ & - Vetor de polarização elétrica (coulomb/metro²) \\
\hline$t$ & - Instante de tempo (segundo) \\
\hline$\varepsilon$ & - Permissividade elétrica (farad/metro) \\
\hline$\Delta t$ & - Passo de discretização temporal (segundo) \\
\hline$\Delta x$ ou $\Delta y$ & - Passo de discretização espacial transversal (metro) \\
\hline$\Gamma$ & - Freqüência de amortecimento \\
\hline$\lambda$ & - Comprimento de onda no espaço-livre (metro) \\
\hline$\mu$ & - Permeabilidade magnética (henry/metro) \\
\hline$\sigma$ & - Condutividade elétrica (siemens/metro) \\
\hline$\sigma^{*}$ & - Perda magnética (ohms/metro) \\
\hline$\omega_{p}$ & - Frequiência de plasma (radiano/segundo) \\
\hline$\omega$ & - Freqüência da Fonte (radiano/segundo) \\
\hline
\end{tabular}




\section{Resumo}

Este trabalho apresenta um novo formalismo bi-dimensional em diferenças finitas no domínio do tempo (2D-FDTD) para a simulação de estruturas baseadas em metamateriais. A natureza dispersiva destes meios é levada em consideração de forma precisa pela inclusão dos modelos materiais de Drude para os tensores permissividade elétrica e permeabilidade magnética. Todos os elementos dos tensores são considerados neste formalismo, o que o torna muito atraente para a modelagem de uma classe geral de estruturas eletromagnéticas. Dois efeitos de enorme impacto são analisados em detalhes, sendo eles a cobertura de invisibilidade e o rotacionamento de campo.

Ambos efeitos requerem a utilização de técnicas de transformação de coordenadas a qual deve ser aplicada apenas na região onde os campos eletromagnéticos precisam ser manipulados, tirando vantagem da invariância das equações de Maxwell quanto a estas operações. Esta técnica redefine localmente os parâmetros de permissividade e permeabilidade do meio transformado.

O formalismo implementado apresentou grande estabilidade e precisão, uma conseqüência direta da natureza dispersiva dos modelos materiais de Drude, o que o caracteriza como uma boa contribuição para uma completa compreensão da fenomenologia por trás destes efeitos fascinantes. Os resultados numéricos apresentaram boa concordância com os disponíveis na literatura. Foi também observado que ambas estruturas são muito sensíveis a variações de frequiência do campo de excitação.

Palavras-chave: Meios dispersivos, FDTD, Metamateriais, Anisotropia. 


\section{Abstract}

This work introduces an extended two-dimensional finite difference time domain method (2D-FDTD) for the simulation of metamaterial based structures. The dispersive nature of these media is accurately taken into account through the inclusion of the Drude material models for the permittivity and permeability tensors. All tensor elements are properly accounted for, making the formalism quite attractive for the modeling of a general class of electromagnetic structures. Two striking effects are investigated with the proposed model, namely, the invisibility cloaking and the field rotation effects.

Both effects require the utilization of a coordinate transformation technique which must be applied only in the region where the electromagnetic field needs to be manipulated, taking advantage of the invariance of Maxwell's equations with respect to these operations. This technique locally redefines the permittivity and permeability parameters of the transformed media.

The implemented formalism has proved to be quite stable and accurate, a direct consequence of the dispersive nature of the Drude material model, which characterizes it as a good contribution to fully understand the phenomenology behind these fascinating effects. The numerical results are in good agreement with those available in the literature. It was also verified that both structures are very sensitive to frequency variations of the excitation field.

Keywords: Dispersive media, FDTD, Metamaterials, Anisotropy. 


\section{Capítulo 1}

\section{Introdução}

Recentemente, a comunidade científica foi brindada com o surgimento de uma nova classe de materiais cujas propriedades constitutivas apresentavam características marcadamente distintas daquelas observadas em materiais ditos convencionais, qual sejam, permissividade elétrica e permeabilidade magnética negativas [1]. Estes novos materiais, por serem constituídos por "átomos" macroscópicos, vêm sendo comumente denominados de metamateriais. O prefixo meta é uma alusão à natureza transcendental de suas inusitadas propriedades eletromagnéticas, como refração negativa, por exemplo. Diferente do que se imagina, o estudo de materiais constituídos de "átomos macroscópicos" (esta definição, assim como a de metamaterial, ficará clara mais à frente), vem ocorrendo há bastante tempo. A primeira tentativa de explorar este conceito ocorreu em 1898, quando Bose [1] realizou o primeiro experimento na faixa de microondas em estruturas com deformações metálicas, as quais são atualmente definidas como meios quirais. Mais tarde, em 1948, Kock [1] projetou lentes de microondas a partir de arranjos metálicos periódicos na forma de esferas, discos e fitas, visando obter estruturas com diferentes índices de refração. No final da década de 60, o cientista russo Victor Veselago investigou teoricamente as características eletrodinâmicas de um meio apresentando tanto a permissividade elétrica $(\varepsilon)$ quanto a permeabilidade magnética $(\mu)$ negativas [2], no que veio a se tornar o marco fundamental na literatura de metamateriais. Uma maneira de se obter materiais com este comportamento consiste, conforme sugerido por Smith et al. [3], na construção de compostos tridimensionais feitos com fios condutores [4]-[5] (para a obtenção de permissividade negativa - mostrado na Fig. 1.1.), e ressoadores em anel segmentado (SRR) [6] (para a obtenção de permeabilidade negativa - mostrado na Fig. 1.2.). Foi 
demonstrado em [5] que o comportamento da estrutura para obtenção de $\varepsilon$ negativo seria semelhante à de um plasma, contanto que o vetor campo elétrico esteja orientado na mesma direção dos fios condutores. Além disso, o valor do índice de refração efetivo desse material é dependente do valor do espaçamento e do raio dos bastões [5].

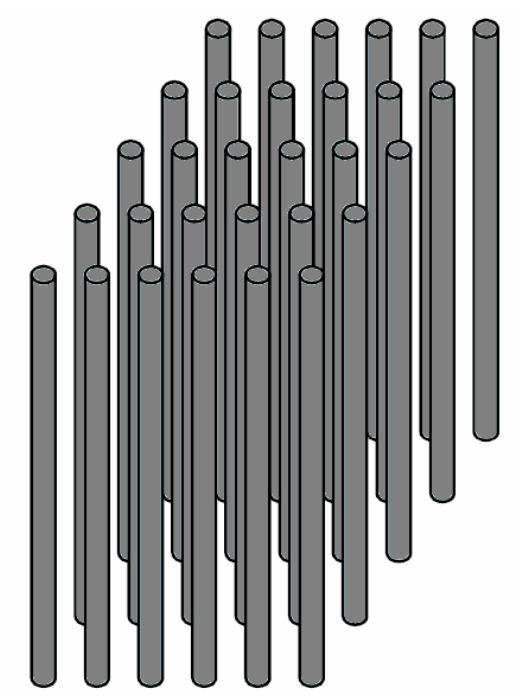

Fig. 1.1 - Arranjo tridimensional de fios condutores para produzir materiais com $\varepsilon$ negativo [4].

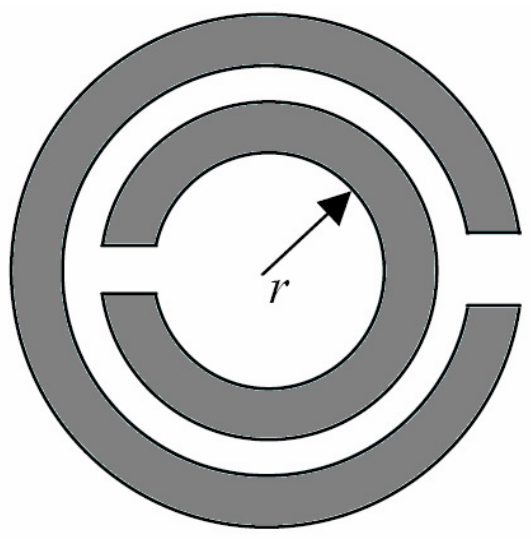

Fig. 1.2 - Ressoador em anel segmentado (SRR) utilizado para obtenção de materiais $\operatorname{com} \mu$ negativo [6].

Tendo em vista que a obtenção de $\varepsilon$ e $\mu$ negativos já podia ser realizada com sucesso de forma individual, Smith et al. [2] realizaram a primeira implementação de 
um material onde estas características estavam presentes simultaneamente. $\mathrm{O}$ metamaterial resultante é apresentado esquematicamente na Fig. 1.3.

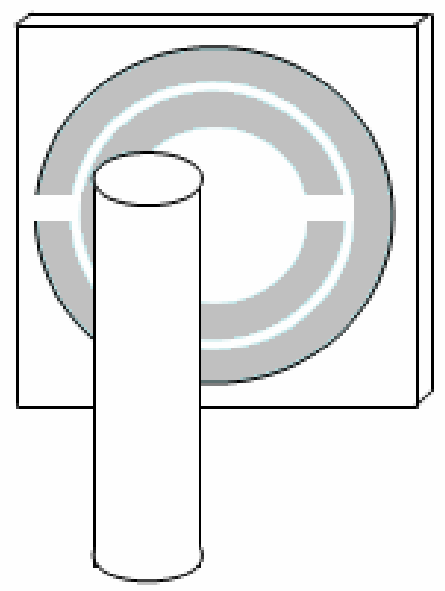

Fig. 1.3 - Representação esquemática do metamaterial proposto em [3].

Entretanto, a anisotropia apresentada por este meio limitou suas propriedades de transmissão (dentro da faixa de freqüências onde $\varepsilon$ e $\mu$ são negativos) a uma determinada polarização e ângulo de incidência. Dois anos mais tarde, Shelby et al. propuseram uma nova abordagem onde SRR's quadrados foram definidos sobre um dos lados de uma placa dielétrica de fibra de vidro, no lado oposto foram gravadas linhas condutoras, conforme ilustra a Fig. 1.4 [7]. Cada placa dielétrica individual foi distribuída em uma rede periódica bidimensional. Deste modo, foi possível obter um meio isotrópico com $\varepsilon$ e $\mu$ negativos na faixa de microondas [7].

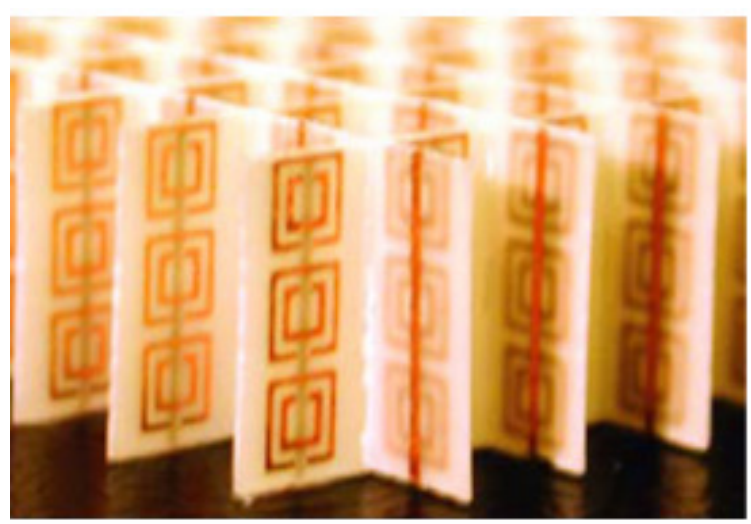

Fig. 1.4 - Estrutura desenvolvida por Shelby et al. em [7] para obter um meio isotrópico com refração negativa. 
É interessante notar que desde o trabalho seminal de Pendry [6], que culminou na fabricação do primeiro metamaterial propriamente dito [2], o volume de publicações neste assunto tem experimentado um acentuado crescimento, como ilustrado na Fig. 1.5. O crescente interesse pelo estudo destes novos meios é justificado pelas respostas eletromagnéticas que estas estruturas proporcionam, possibilitando o desenvolvimento de dispositivos com promissoras funcionalidades eletromagnéticas em diversas áreas tecnológicas, tais como, aeroespacial, telecomunicações, engenharia biomédica etc. [8].

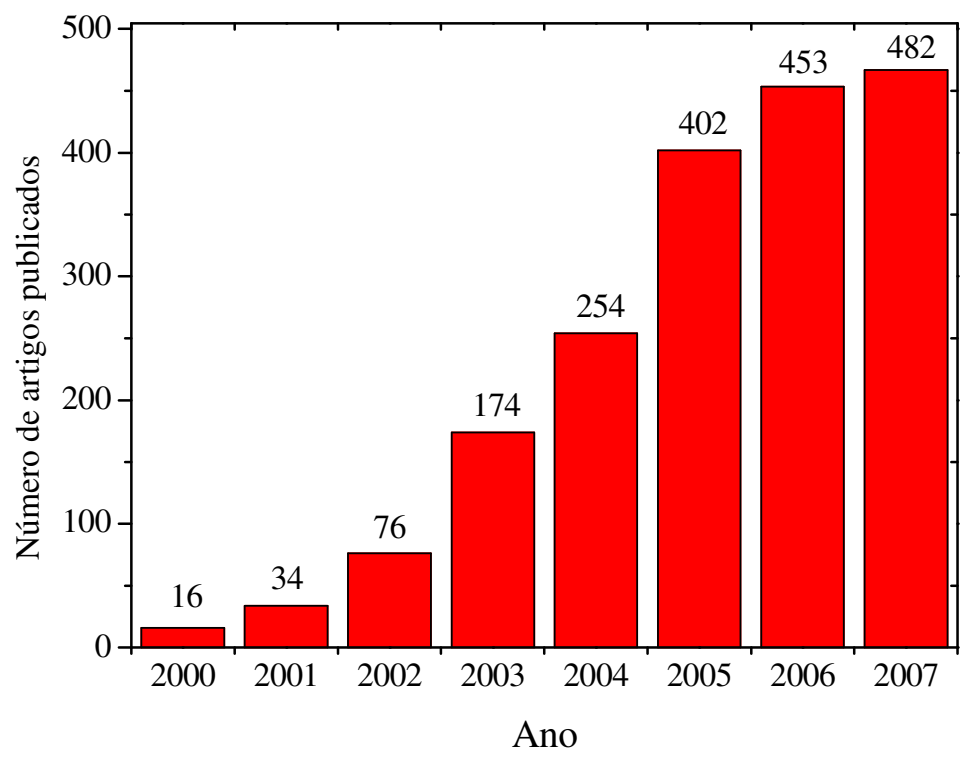

Fig. 1.5 - Números de artigos publicados sobre metamateriais nos últimos anos, obtidos do ISI [9].

Um aspecto importante a ser observado está relacionado à denominação do material mostrado na Fig. 1.4. Como originalmente previsto por Veselago [2], a propagação das ondas eletromagnéticas nos materiais $\operatorname{com} \varepsilon$ e $\mu$ negativos faz-se no sentido oposto à dos meios convencionais. Com isso, o vetor campo elétrico $\vec{E}$, o vetor campo magnético $\vec{H}$ e o vetor de onda $\vec{k}$ obedecem à regra da mão esquerda nos meios analisados por Veselago. Consequëntemente, uma onda eletromagnética ao incidir sobre este material sofrerá refração negativa. Por este motivo, Veselago denominou esses materiais de LHM, ou materiais que obedecem à regra da mão esquerda (left-handed materials), em oposição aos materiais que obedecem à regra da mão direita - RHM (right-handed materials). No entanto, por essa denominação não fazer muito sentido no 
idioma russo (de acordo com aquele pesquisador), esses meios foram por ele denominados de meios com índice de refração negativo - NIM (negative index media).

Outras denominações também têm surgido na literatura, tais como meios de propagação reversa (backward wave media), ou ainda, meio de Veselago, uma referência aos meios isotrópicos com seus parâmetros eletromagnéticos $(\varepsilon$ e $\mu)$ negativos. Entretanto, a denominação metamaterial é a mais utilizada na comunidade científica. Isto é justificado pela forma como estes materiais são obtidos (através de um arranjo tridimensional de "átomos" macroscópicos) e também devido à excentricidade de seus parâmetros eletromagnéticos, como será esclarecido posteriormente. Até hoje não se chegou a um consenso sobre o que realmente seriam estes materiais. Sihvola [10]-[11] questiona esta denominação e faz uma necessária análise etimológica da palavra metamaterial, salientando que o prefixo "meta", oriundo do idioma grego, quando combinado com nomes, verbos ou adjetivos, enfatiza uma mudança de posição, de formato, como, por exemplo, em metamorfose. Além disso, esse prefixo também significa elevado, sublime, máximo, como, em metafísico. Deste modo, Sihvola concluiu que o prefixo "meta" enfatiza o aspecto transcendental da palavra. Contudo, o autor também constatou que o uso da palavra metamaterial com diferentes conotações, afetou o foco principal desse termo, conforme pode ser observado nos dois exemplos seguintes:

No sítio do instituto virtual de pesquisas em materiais eletromagnéticos artificiais Metamorphose [12], encontra-se a seguinte definição:

Metamaterial é um arranjo estrutural de elementos artificiais, desenvolvidos para proporcionar propriedades eletromagnéticas vantajosas e não usuais.

Já o departamento de defesa norte-americano, DARPA, utiliza a seguinte definiç̧ão [13]:

MetaMaterial é uma nova classe de nano-inclusões artificiais ordenadas numa estrutura que apresenta propriedades excepcionais não observadas na natureza. Estas propriedades também não são observadas nos materiais constituintes dessas estruturas e resultam das respostas eletromagnéticas dessas inclusões extrínsecas, nãohomogêneas e fabricadas artificialmente. 
Apesar das definições serem bastante subjetivas, dois tópicos essenciais entre elas podem ser distinguidos. Metamateriais devem apresentar propriedades [10]: i) não observadas nos materiais que os constituem e ii) não observadas na natureza. $\mathrm{O}$ item (i) não enfatiza o caráter microscópico dos materiais que constituem o metamaterial, mas sim, somente considera que no composto há materiais homogêneos com parâmetros eletromagnéticos bem definidos, o que seria suficiente para a obtenção de $\varepsilon$ e $\mu$ do metamaterial. O segundo item enfatiza o aspecto de que os metamateriais são estruturas desenvolvidas para uma determinada função, sendo projetados porque não há, na natureza, materiais que atendam a tal função.

Diante do que foi acima exposto, a definição largamente difundida na literatura será adotada neste trabalho, descrita a seguir: Metamateriais são arranjos (periódicos ou não) de inclusões (ou “átomos") macroscópicas apresentando dimensões muito menores que o comprimento de onda em questão. Adicionalmente, estes materiais apresentam parâmetros constitutivos definidos em função da resposta coletiva destas inclusões a uma dada incidência de onda eletromagnética.

Embora os metamateriais tenham sido originalmente idealizados visando a obtenção de $\varepsilon$ e $\mu$ negativos, sua funcionalidade vai além dessa característica, já que permitem qualquer combinação possível de sinais entre esses dois parâmetros, como ilustra na Fig. 1.6. Nesta figura, o material que apresenta tanto a permissividade elétrica quanto a permeabilidade magnética positivas é denominado meio duplo-positivo (DPSdouble-positive medium) e inclui os materiais dielétricos convencionais nessa classificação. O segundo quadrante da Fig.1.6 é reservado ao meio que apresenta $\varepsilon<0$ e $\mu>0$, o qual é denominado de meio $\varepsilon$-negativo (ENG-epsilon-negative medium). Certos metais apresentam esse comportamento quando iluminados por ondas eletromagnéticas na faixa do infravermelho e do espectro visível. Além destes materiais, em [5], Pendry desenvolveu uma estrutura com esses parâmetros eletromagnéticos na faixa de microondas. Material que apresenta $\varepsilon>0$ e $\mu<0$ é denominado de meio $\mu$-negativo (MNG-mu-negative medium). Para uma determinada faixa de freqüências, os meios girotrópicos pertencem a essa classificação. Além disso, uma estrutura com tais parâmetros foi proposta por Pendry em [6] para a faixa de microondas. Já o meio pertencente ao terceiro quadrante da Fig. 1.6, apresenta simultaneamente, para uma mesma faixa de freqüência, $\varepsilon$ e $\mu$ negativos, sendo denominado de meio duplo-negativo (DNG-double-negative medium). Meios com esta característica não são diretamente encontrados na natureza, e só podem ser obtidos através de metamateriais. Um meio 
com estes parâmetros apresenta índice de refração negativo, o que implica em uma velocidade de grupo anti-paralela à velocidade de fase, causando interessantes efeitos, como, por exemplo, uma inversão de direção no efeito Doppler ou uma inversão do raio refratado na Lei de Snell comparados aos meios convencionais. Além disso, os metamateriais também podem ser implementados para apresentarem os mesmos parâmetros eletromagnéticos dos materiais pertencentes aos outros três quadrantes.

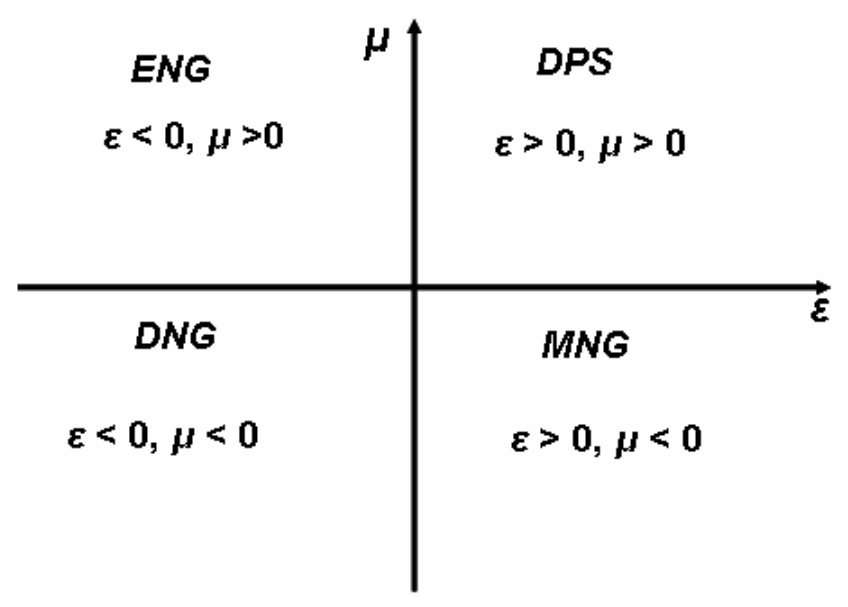

Fig. 1.6 - Classificação dos materiais combinando seus parâmetros eletromagnéticos.

Características como as descritas acima têm possibilitado o desenvolvimento de uma vasta gama de aplicações para esses materiais. Entre elas podemos citar linhas de transmissões apresentando característica excêntrica de dispersão quando comparadas às linhas convencionais, conforme apresentado em [14]. O controle da dispersão é uma importante característica no desenvolvimento de estruturas compactas, tais como, deslocadores de fase e linhas de atrasos, utilizadas em sistemas de comunicações [15]. A combinação de linhas LHM e RHM, mostrada na Fig. 1.7, permite controlar simultaneamente a impedância característica e a constante de fase da estrutura, proporcionando um casamento numa larga faixa de freqüência [16]. 


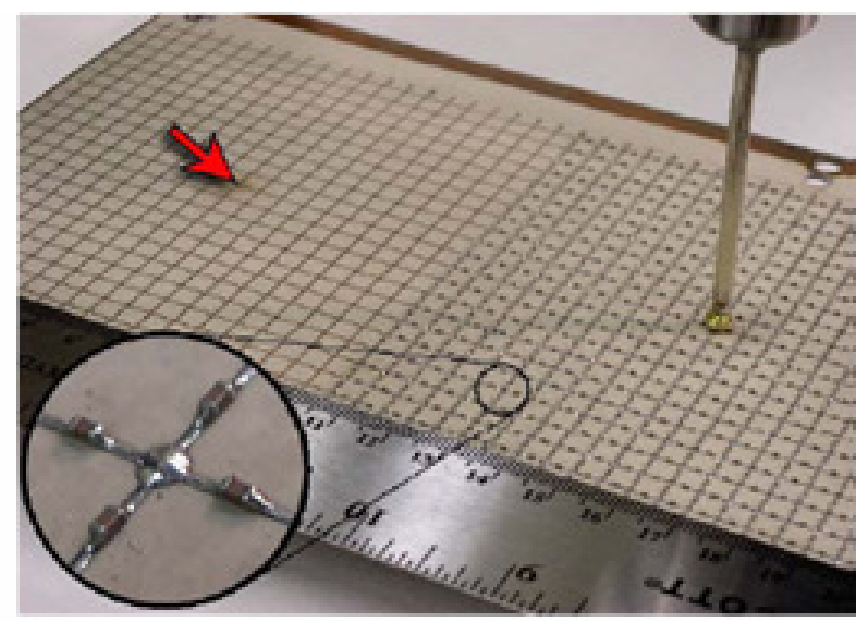

Fig. 1.7 - Combinação de células 2D de linhas RHM (flecha) e LHM [15].

Metamateriais têm também sido utilizados na otimização de antenas, como demonstrado em [15]. A utilização desses materiais em antena convencional melhorou em mais de $6 \mathrm{~dB}$ o ganho de tal dispositivo apresentando uma eficiência próxima a $100 \%$.

Em [17], um grupo formado por cientistas do College Boston e da Duke Univerty analisaram e fabricaram uma estrutura, conforme mostrada na Fig. 1.8, capaz de absorver quase $100 \%$ da radiação incidente para uma estreita faixa de freqüência. Diferente dos absorvedores convencionais, esta estrutura é construída exclusivamente de elementos metálicos, proporcionando ao dispositivo uma maior flexibilidade para aplicações relacionadas com a detecção ou filtragem da radiação incidente. 


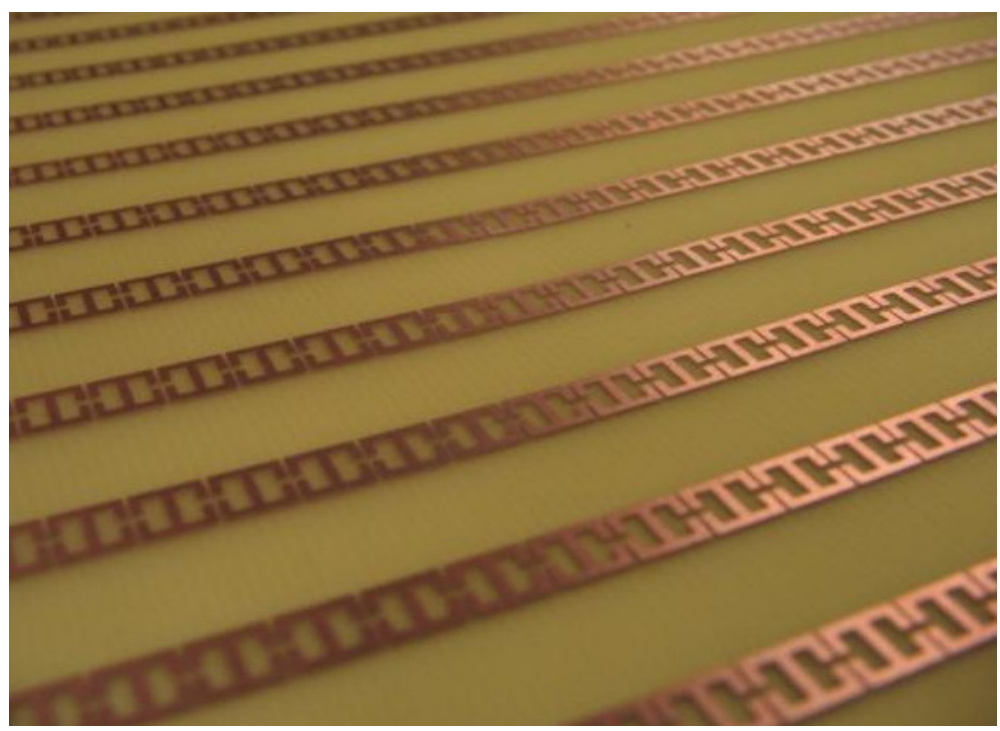

Fig. 1.8 - Absorvedor baseado em metamateriais, desenvolvido em [17].

Uma aplicação que vem ganhando destaque desde que foi originalmente proposta em 2006 por Pendry et al. [18] (devido à sua surpreendente característica de funcionamento) é a cobertura de invisibilidade eletromagnética, uma estrutura desenvolvida a partir de metamaterial capaz de reduzir o espalhamento de um objeto na faixa de microondas. Em 2006, Pendry et al. [19], apresentaram um trabalho onde sugeriam a manipulação de campos eletromagnéticos utilizando metamateriais. Ainda neste mesmo ano, Schurig et al. [18] projetaram e analisaram experimentalmente o primeiro dispositivo baseado em metamaterial capaz de proporcionar a invisibilidade eletromagnética, conforme apresentado na Fig. 1.9. 


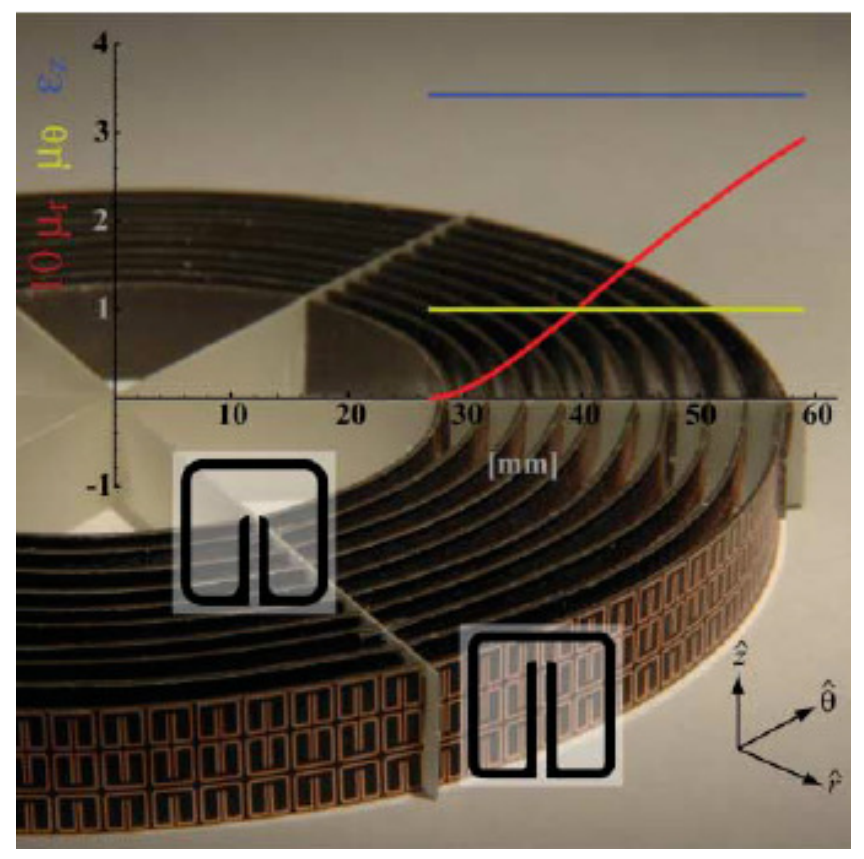

Fig. 1.9 - Cobertura de invisibilidade eletromagnética construída com metamateriais [18].

Em outubro daquele mesmo ano, Schurig et al. [20] utilizaram a técnica de transformação de coordenadas (tirando vantagem da invariância das equações de Maxwell na transformação espaço-tempo) para a determinação de novos parâmetros constitutivos que possibilitassem a manipulação dos campos eletromagnéticos em uma dada estrutura como desejado. Nesta análise, uma dada região do espaço livre foi redistribuída dentro de um novo espaço com volume diferente do anterior e com seus novos parâmetros constitutivos apresentando variação espacial e anisotropia [20]. Esses novos parâmetros constitutivos, obtidos via transformação de coordenadas, se comportam exatamente como o meio original (nesse caso, o espaço livre) permitindo, assim, um perfeito casamento de impedância entre os dois meios. Desde então, essa metodologia vem ganhando ênfase entre os grupos de pesquisas de materiais eletromagnéticos, pois ela fornece os parâmetros constitutivos de estruturas complexas capazes de manipular a onda incidente de maneira simples e precisa. Em março de 2007, Cummer et al. [21] analisaram numericamente uma cobertura de invisibilidade eletromagnética de formato cilíndrico, considerando parâmetros eletromagnéticos tanto ideais quanto não-ideais (mas fisicamente realizáveis). Em abril de 2007, Cai et al. [22] publicaram suas análises teóricas para o desenvolvimento desses dispositivos na faixa óptica do espectro de freqüência [22]. Além das coberturas de invisibilidade, outras 
estruturas capazes de manipular a onda eletromagnética foram também propostas, tais como hiperlentes [23]-[24], concentradores [25]-[26], e rotacionadores de campo eletromagnético (onde a frente de onda é rotacionada por um ângulo pré-estabelecido) [27]-[28].

Conforme exposto acima, uma grande repercussão no meio científico ocorreu após as publicações [18]-[31], pois vários dispositivos capazes de manipular a onda eletromagnética puderam ser desenvolvidos baseados na metodologia adotada naquelas publicações. No entanto, foi observado que em todos esses trabalhos o caráter dispersivo dos metamateriais não foi levado em consideração. Todo material apresenta alguma dispersão, sendo que alguns meios podem apresentar este efeito mais acentuado do que outros (caso dos metamateriais). É indiscutível que a análise da propagação de ondas eletromagnéticas é tão precisa quanto mais próximo da realidade são os modelos utilizados. No caso dos metamateriais, os modelos mais adequados para a inclusão de efeitos dispersivos são os modelos materiais de Drude, Debye e de Lorentz [32]. Para efeito de modelagem numérica, o modelo de Drude tem encontrado grande aplicação em virtude apresentar uma maior banda de operação [1].

\section{1-Contribuições deste trabalho}

Este trabalho se propõe a estender o formalismo numérico normalmente utilizado na literatura de forma que os efeitos dispersivos do metamaterial sejam corretamente considerados. Para isso, será considerado o modelo material de Drude, por apresentar uma maior banda de operação reduzindo, assim, o custo computacional das simulações.

É importante ressaltar que o modelo de Lorentz pode igualmente ser utilizado, o que deverá ser realizado em trabalhos futuros. O formalismo adotado para este fim é o de diferenças finitas bidimensional no domínio do tempo (2D-FDTD). Neste contexto, serão investigadas duas estruturas baseadas em metamateriais e capazes de realizar a manipulação dos campos eletromagnéticos, qual sejam, a cobertura de invisibilidade e o rotacionador de campo eletromagnético. Tanto o formalismo proposto quanto os resultados obtidos são discutidos no decorrer deste trabalho. 


\title{
1.2-Organização do texto
}

\begin{abstract}
Este trabalho está organizado da seguinte forma: O Capítulo 2 aborda o formalismo proposto por esta dissertação, mostrando a obtenção dos parâmetros constitutivos do material responsável pela manipulação da radiação incidente. Adicionalmente, esse capítulo aborda a modelagem de meios dispersivos e descreve, ainda, os principais aspectos do método 2D-FDTD. No Capítulo 3, o formalismo desenvolvido é aplicado na simulação de estruturas capazes de manipular a radiação incidente. Nesse capítulo são analisadas duas estruturas, quais sejam, a cobertura de invisibilidade eletromagnética e o rotacionador de campo. Posteriormente, são descritas as conclusões e as propostas para trabalhos futuros. Nos apêndices A, B e C são abordados, respectivamente, os seguintes tópicos: cálculo dos parâmetros eletromagnéticos da cobertura de invisibilidade, fluxograma do algoritmo FDTD e condições de contorno absorventes na borda computacional.
\end{abstract}




\section{Capítulo 2}

\section{Formalismo para equações de diferenças finitas no domínio do tempo}

\section{Introdução}

Conforme descrito no capítulo anterior, as pesquisas envolvendo materiais artificiais iniciaram-se no final do século XIX, mas ganharam destaque no ano 2000, quando Smith, Shelby e outros pesquisadores [5],[7] comprovaram experimentalmente as previsões de Veselago [2] sobre a resposta eletromagnética de meios com índice de refração negativa. Desde então, vários dispositivos com interessantes funcionalidades puderam ser desenvolvidos utilizando metamateriais [18]-[31], dentre eles a cobertura de invisibilidade [18]. No entanto, esses trabalhos não levaram em consideração a natureza dispersiva dos metamateriais, a qual é normalmente descrita em termos dos modelos materiais de Drude e/ou Lorentz [1]. Sendo assim, o presente trabalho tem por objetivo incorporar esta importante característica por meio do modelo de Drude (por este apresentar uma maior largura de banda que o de Lorentz) em um formalismo bidimensional baseado em diferenças finitas no domínio do tempo (2D-FDTD).

Na seção seguinte é apresentada a modelagem matemática do problema analisado. Na Seção 2.2, a modelagem de meios dispersivos é abordada. A Seção 2.3 descreve os aspectos mais relevantes do método 2D-FDTD para a modelagem de estruturas baseadas em metamateriais. 


\section{1-Modelagem matemática}

A geometria investigada neste trabalho é apresentada esquematicamente na Fig. 2.1. O objetivo é simular a propagação de ondas eletromagnéticas através desta estrutura visando duas aplicações distintas: 1) cobertura de invisibilidade, e 2) rotacionamento de campo. As equações a seguir descrevem em detalhes o formalismo matemático com foco no primeiro caso, uma vez que a segunda aplicação nada mais é que uma variação da primeira.

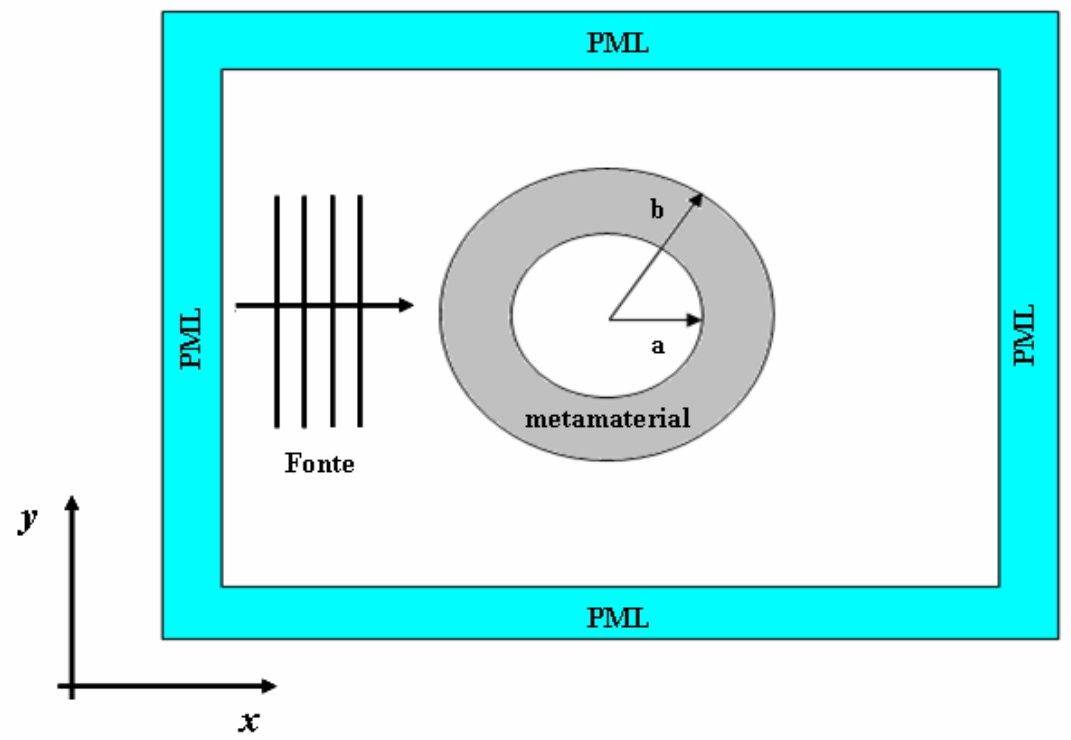

Fig. 2.1 - Domínio computacional analisado neste trabalho.

O ponto de partida para esta investigação são as equações de Maxwell, que podem ser escritas da seguinte forma:

$$
\frac{\partial D_{x}}{\partial t}=\frac{\partial H_{z}}{\partial y}
$$




$$
\begin{gathered}
\frac{\partial D_{y}}{\partial t}=-\frac{\partial H_{z}}{\partial x}, \\
\frac{\partial B_{z}}{\partial t}=\left[\frac{\partial E_{x}}{\partial y}-\frac{\partial E_{y}}{\partial x}\right],
\end{gathered}
$$

para modos $\mathrm{TE}_{\mathrm{z}}$, e

$$
\begin{gathered}
\frac{\partial B_{x}}{\partial t}=-\frac{\partial E_{z}}{\partial y}, \\
\frac{\partial B_{y}}{\partial t}=\frac{\partial E_{z}}{\partial x}, \\
\frac{\partial D_{z}}{\partial t}=\left[\frac{\partial H_{y}}{\partial x}-\frac{\partial H_{x}}{\partial y}\right],
\end{gathered}
$$

para modos $\mathrm{TM}_{\mathrm{z}}$, onde:

$\vec{E}$ é o vetor campo elétrico $(\mathrm{V} / \mathrm{m})$,

$\vec{D}$ é o vetor deslocamento elétrico $\left(\mathrm{C} / \mathrm{m}^{2}\right)$,

$\vec{H}$ é o vetor campo magnético $(\mathrm{A} / \mathrm{m})$, e

$\vec{B}$ é o vetor densidade de fluxo magnético $\left(\mathrm{wb} / \mathrm{m}^{2}\right)$.

Adicionalmente, temos as relações constitutivas, as quais relacionam os vetores de campo elétrico e magnético aos vetores deslocamento elétrico e densidade de fluxo magnético, respectivamente, através dos parâmetros do meio, ou seja,

$$
\begin{aligned}
& \tilde{\vec{D}}=\varepsilon_{0} \bar{\varepsilon}_{r}(\omega) \tilde{\vec{E}}, \\
& \widetilde{\vec{B}}=\mu_{0} \bar{\mu}_{r}(\omega) \tilde{\vec{H}},
\end{aligned}
$$


onde:

$\varepsilon_{0}$ é a permissividade elétrica do espaço-livre $(\mathrm{F} / \mathrm{m})$,

$\mu_{0}$ é a permeabilidade magnética do espaço-livre (H/m),

$\overline{\bar{\varepsilon}}_{r}$ é o tensor permissividade elétrica relativa, e

$\bar{\mu}_{r}$ é o tensor permeabilidade magnética relativa.

O sobrescrito “ " indica que está sendo considerado o domínio da freqüência.

Em se tratando de projeto de uma cobertura de invisibilidade, o objetivo é reduzir ao máximo o espalhamento total de campo eletromagnético produzido por um determinado objeto. Isso contrasta com as técnicas de camuflagens existentes, uma vez que estas absorvem ou reduzem o espalhamento eletromagnético da onda incidente somente em uma única direção. O desempenho adequado destes dispositivos passa, fundamentalmente, pela escolha apropriada dos parâmetros dos materiais envolvidos. Como esses materiais apresentam características específicas, torna-se necessário definir como estes devem ser obtidos para depois dar continuidade à expansão das equações de Maxwell.

Portanto, considere um objeto (também denominado de espalhador) situado no espaço-livre e uma frente de ondas planas propagando-se através deste meio. Uma interação típica entre o objeto e a onda é apresentada no esquema ilustrativo da Fig. 2.2. Nesta figura, a frente de onda incidente sofre interferência ao encontrar o objeto (espalhador) e uma parcela de sua energia, representada pelas setas em volta do objeto, é espalhada, causando uma deformação na frente de ondas planas.

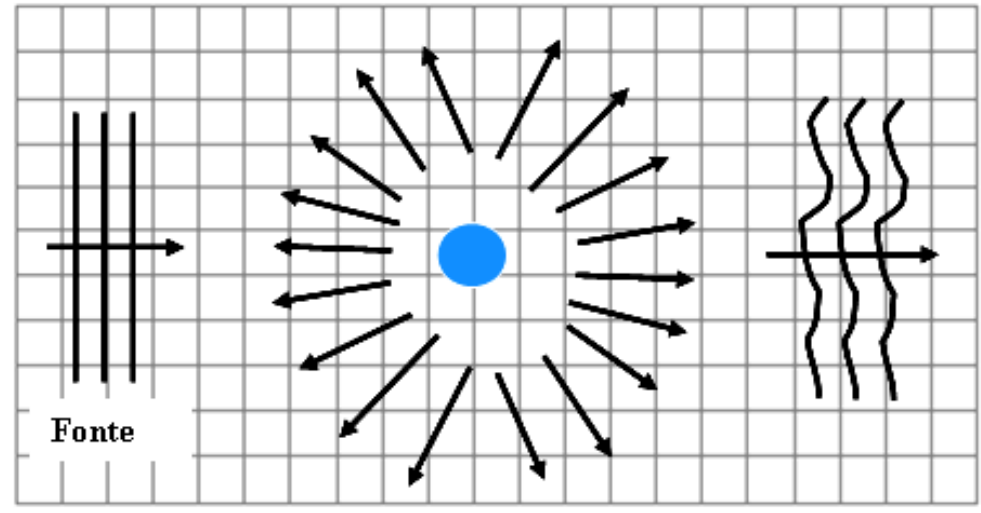

Fig. 2.2 - Esquema ilustrativo, no plano cartesiano, da interação de um objeto com a radiação incidente gerada por uma fonte de ondas planas. 
Uma forma de evitar que a onda interaja com o objeto e, consequientemente, não seja refletida ou espalhada, consiste em redirecionar a frente de ondas planas de forma que ela contorne o objeto sem que haja espalhamento. Isto pode ser feito levando-se em consideração a invariância das equações de Maxwell a qualquer transformação espaçotempo [33]. Sendo assim, é possível utilizar técnicas, como as de transformação de coordenadas, de forma a se expandir a origem do problema (a origem é suposta por conveniência no ponto onde está localizado o espalhador) criando-se uma região (círculo de raio $a$ ) em cujo interior não pode haver a propagação de qualquer onda eletromagnética, conforme ilustra a Fig. 2.3. Para todos os efeitos, esta região não é mais material. Por conseguinte, qualquer objeto disposto dentro dela não irá interagir com a onda e, assim, não produzirá qualquer espalhamento.

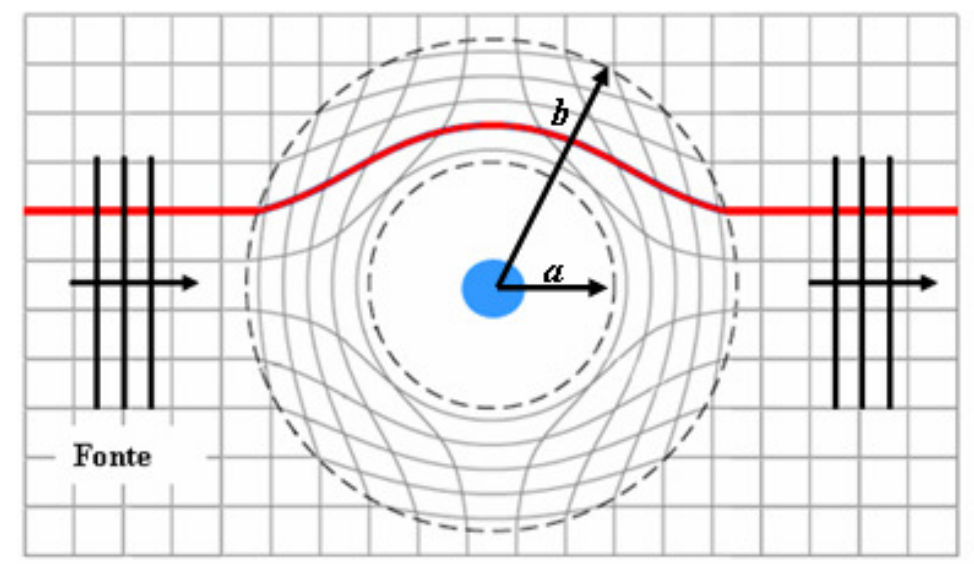

Fig. 2.3 - Esquema ilustrativo da criação de uma região nula à radiação eletromagnética, obtida a partir da expansão da origem do sistema cartesiano. A linha destacada, apenas indica o efeito da deformação do meio na trajetória de um feixe.

Deste modo, e com base na Fig. 2.4, para $0 \leq r \leq b$ (antes da transformação, onde $b$ é o raio da região de fronteira entre os dois sistemas) e $a \leq r^{\prime} \leq b$ (após a transformação, onde $a$ é o raio da expansão da origem) a seguinte relação pode ser obtida:

$$
r=0 \Leftrightarrow r^{\prime}=a
$$

Esta relação apenas indica a origem para os dois casos, ou seja, $r=0$ para o sistema não transformado, e $r^{\prime}=a$ para o sistema transformado. 


$$
r=b \Leftrightarrow r^{\prime}=b
$$

Esta relação, por sua vez, indica a fronteira de ambos os sistemas que neste caso apresentam, necessariamente, o mesmo raio $b$.

Assim, é possível obter de forma direta uma equação relacionando ambos os sistemas (transformado e não-transformado), válida para $0 \leq r \leq b$, ou seja,

$$
r^{\prime}=\frac{b-a}{b} r+a
$$

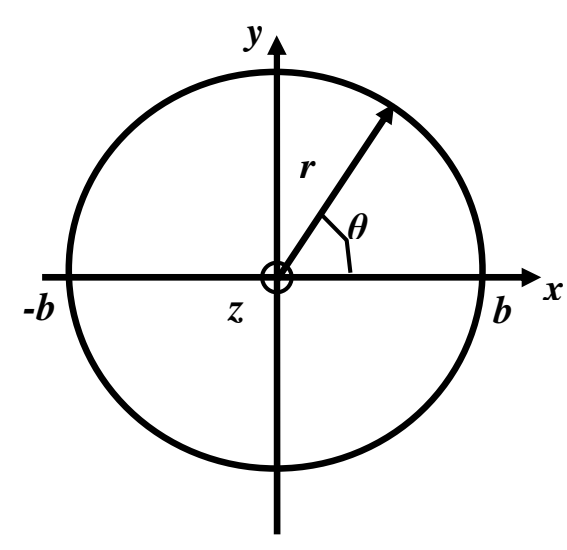

(a)

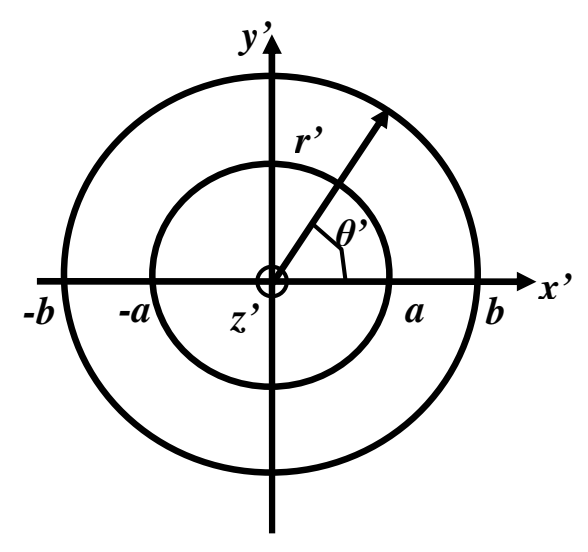

(b)

Fig. 2.4 - Representação no sistema cartesiano. (a) antes da transformação e (b) após a transformação.

Portanto, a região onde os campos eletromagnéticos deverão ser adequadamente manipulados é dada pelo cilindro ilustrado na Fig. 2.4 (b). A maneira mais conveniente de se obter esta manipulação consiste na utilização de meios metamateriais, tendo em vista a facilidade que esses meios apresentam quanto à alteração de seus parâmetros constitutivos [18]-[21]. É importante observar que as características de propagação eletromagnética na região transformada (cilindro da Fig. 2.4(b)) são, necessariamente, as mesmas do sistema não-transformado (Fig. 2.4(a)). Isso se deve à invariância das equações de Maxwell quanto a estas transformações. Seguindo a definição de Post em 
[33], os parâmetros constitutivos do material na região transformada, obedecem a uma lei homogênea e linear de transformação, dada por:

$$
\begin{aligned}
& \mathcal{E}_{r}^{i^{\prime} j^{\prime}}(\omega)=\left|\operatorname{det}\left(\Lambda_{\alpha}^{\alpha^{\prime}}\right)\right|^{-1} \Lambda_{i}^{i^{\prime}} \Lambda_{j}^{j^{\prime}} \varepsilon_{r}^{i j}(\omega), \\
& \mu_{r}^{i^{\prime} j^{\prime}}(\omega)=\left|\operatorname{det}\left(\Lambda_{\alpha}^{\alpha^{\prime}}\right)\right|^{-1} \Lambda_{i}^{i^{\prime}} \Lambda_{j}^{j^{\prime}} \mu_{r}^{i j}(\omega),
\end{aligned}
$$

onde:

$\Lambda_{\alpha}^{\alpha^{\prime}}$ é a matriz jacobiana [33], que relaciona o espaço original com o espaço transformado, sendo definida por:

$$
\Lambda_{\alpha}^{\alpha^{\prime}}=\left[\begin{array}{lll}
\Lambda_{1}^{1^{\prime}} & \Lambda_{2}^{1^{\prime}} & \Lambda_{3}^{1^{\prime}} \\
\Lambda_{1}^{2^{\prime}} & \Lambda_{2}^{2^{\prime}} & \Lambda_{3}^{2^{\prime}} \\
\Lambda_{1}^{3^{\prime}} & \Lambda_{2}^{3^{\prime}} & \Lambda_{3}^{3^{\prime}}
\end{array}\right]=\left[\begin{array}{lll}
\frac{\partial x^{\prime}}{\partial x} & \frac{\partial x^{\prime}}{\partial y} & \frac{\partial x^{\prime}}{\partial z} \\
\frac{\partial y^{\prime}}{\partial x} & \frac{\partial y^{\prime}}{\partial y} & \frac{\partial y^{\prime}}{\partial z} \\
\frac{\partial z^{\prime}}{\partial x} & \frac{\partial z^{\prime}}{\partial y} & \frac{\partial z^{\prime}}{\partial z}
\end{array}\right]
$$

$\Lambda_{i, j}^{i^{\prime}, j^{\prime}}$ representam os elementos desta matriz $(i, j=1,2,3)$, e $\varepsilon_{r}^{i j}$ e $\mu_{r}^{i j}$ representam as componentes do tensor permissividade elétrica relativa e permeabilidade magnética relativa, respectivamente, do material não-transformado.

Com o propósito de demonstrar a obtenção da matriz jacobiana, o cálculo do seu primeiro elemento, ou seja, $\Lambda_{1}^{1}$ será apresentado a seguir (todos os outros elementos podem ser obtidos de forma análoga). Com base na Fig. 2.5, a representação do sistema de coordenadas cilíndricas no sistema cartesiano é:

$$
\begin{gathered}
x=r \cos \theta, \\
y=r \sin \theta, \\
r=\sqrt{x^{2}+y^{2}},
\end{gathered}
$$




$$
z=z
$$

Da mesma forma, é possível obter relações similares para a região transformada, ou seja:

$$
\begin{array}{r}
x^{\prime}=r^{\prime} \cos \theta^{\prime}, \\
y^{\prime}=r^{\prime} \sin \theta^{\prime}, \\
r^{\prime}=\sqrt{x^{\prime 2}+y^{\prime 2}}, \\
z^{\prime}=z^{\prime} .
\end{array}
$$

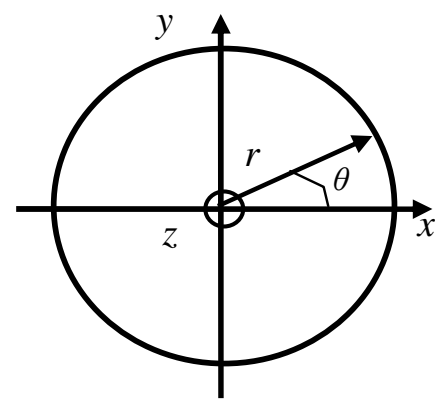

Fig. 2.5 - Representação do sistema de coordenadas cilíndrica no sistema cartesiano.

A relação entre $r$ ' e $r$ foi definida em (2.9) com base na Fig. 2.4. Ainda com relação a esta figura, é possível relacionar as outras duas componentes do sistema de coordenadas cilíndricas original com o da região espacialmente transformada, dadas por:

$$
\begin{gathered}
\theta^{\prime}=\theta, \\
z^{\prime}=z .
\end{gathered}
$$

Isto porque tanto o meio transformado quanto o não-transformado não apresentam alteração com respeito a estas coordenadas. Deste modo, a substituição de (2.9) e (2.18) em (2.14), resulta em:

$$
x^{\prime}=\frac{b-a}{b} x+a \frac{x}{r} .
$$


Logo, o primeiro elemento da matriz jacobiana, $\Lambda_{1}^{1^{\prime}}=\frac{\partial x^{\prime}}{\partial x}$, é:

$$
\begin{gathered}
\frac{\partial x^{\prime}}{\partial x}=\frac{\partial\left(\frac{b-a}{b} x\right)}{\partial x}+\frac{\partial\left(\frac{a x}{r}\right)}{\partial x}= \\
=\frac{b-a}{b}+\frac{a}{r}-\frac{a x \cos \theta}{r^{2}}= \\
=\frac{b-a}{b}+\frac{a}{r}-\frac{a}{r} \cos ^{2} \theta= \\
\frac{\partial x^{\prime}}{\partial x}=\frac{b-a}{b}+\frac{a}{r} \sin ^{2} \theta .
\end{gathered}
$$

O cálculo de todos os elementos da matriz jacobiana é apresentado em mais detalhes no Apêndice A.

Supondo que a cobertura de invisibilidade desejada esteja mergulhada no espaço livre, tem-se que a permissividade elétrica relativa é igual a permeabilidade magnética relativa. Assim, a expressão dos parâmetros eletromagnéticos do metamaterial colocado na região transformada da Fig. 2.3 é:

$$
\overline{\bar{\varepsilon}}_{r}^{\prime}=\overline{\bar{\mu}}_{r}^{\prime}=\left[\begin{array}{ccc}
\frac{r^{\prime}-a}{r^{\prime}} \cos ^{2} \theta+\frac{r^{\prime}}{r^{\prime}-a} \sin ^{2} \theta & \frac{a^{2}-2 r^{\prime} a}{r^{\prime}\left(r^{\prime}-a\right)} \cos \theta \sin \theta & 0 \\
\frac{a^{2}-2 r^{\prime} a}{r^{\prime}\left(r^{\prime}-a\right)} \cos \theta \sin \theta & \frac{r^{\prime}-a}{r^{\prime}} \sin ^{2} \theta+\frac{r^{\prime}}{r^{\prime}-a} \cos ^{2} \theta & 0 \\
0 & 0 & \left(\frac{r^{\prime}-a}{r^{\prime}}\right) \cdot\left(\frac{b}{b-a}\right)^{2}
\end{array}\right] .
$$

De acordo com (2.9), os parâmetros eletromagnéticos desse dispositivo são anisotrópicos, além de apresentarem dependência radial. Fora dessa estrutura, $\varepsilon$ e $\mu$ correspondem aos parâmetros do meio externo, que neste trabalho é o espaço-livre. Isso quer dizer que (tomando por base a Fig. 2.4): 


$$
\begin{array}{r}
r^{\prime}>=b \therefore\left\{\begin{array}{l}
r^{\prime}=r \\
\theta^{\prime}=\theta, \\
z^{\prime}=z
\end{array}\right. \\
a<r^{\prime}<b \therefore\left\{\begin{array}{l}
r^{\prime}=\frac{b-a}{b} r+a \\
\theta^{\prime}=\theta \\
z^{\prime}=z
\end{array}\right.
\end{array}
$$

Uma vez que os parâmetros constitutivos são agora conhecidos, podemos dar continuidade à expansão das equações de Maxwell. Sendo assim, substituindo (2.20) em (2.7) e (2.8), obtém-se:

$$
\begin{aligned}
& {\left[\begin{array}{c}
\tilde{D}_{x} \\
\tilde{D}_{y} \\
\tilde{D}_{z}
\end{array}\right]=\varepsilon_{0}\left[\begin{array}{ccc}
\varepsilon_{r_{x x}} & \varepsilon_{r_{x y}} & 0 \\
\varepsilon_{r y x} & \varepsilon_{r_{y y}} & 0 \\
0 & 0 & \varepsilon_{r_{z z}}
\end{array}\right]\left[\begin{array}{c}
\tilde{E}_{x} \\
\tilde{E}_{y} \\
\tilde{E}_{z}
\end{array}\right],} \\
& {\left[\begin{array}{c}
\tilde{B}_{x} \\
\tilde{B}_{y} \\
\tilde{B}_{z}
\end{array}\right]=\mu_{0}\left[\begin{array}{ccc}
\mu_{r_{x x}} & \mu_{r x y} & 0 \\
\mu_{r_{y x}} & \mu_{r_{y y}} & 0 \\
0 & 0 & \mu_{r_{z z}}
\end{array}\right]\left[\begin{array}{c}
\tilde{H}_{x} \\
\tilde{H}_{y} \\
\tilde{H}_{z}
\end{array}\right] .}
\end{aligned}
$$

De posse de (2.21) e (2.22), além de considerar a dependência temporal dos vetores de campo elétrico e magnético da forma $e^{-j \omega t}$, calcula-se a transformada de Fourier das equações (2.1)-(2.3) e (2.4)-(2.6), estas que são reescritas como:

$$
\begin{aligned}
& -j \omega \tilde{D}_{x}=-j \omega \varepsilon_{0} \varepsilon_{r x x} \tilde{E}_{x}-j \omega \varepsilon_{0} \varepsilon_{r x y} \tilde{E}_{y}=\frac{\partial \tilde{H}_{z}}{\partial y}, \\
& -j \omega \tilde{D}_{y}=-j \omega \varepsilon_{0} \varepsilon_{r y x} \tilde{E}_{x}-j \omega \varepsilon_{0} \varepsilon_{r y y} \tilde{E}_{y}=-\frac{\partial \tilde{H}_{z}}{\partial x},
\end{aligned}
$$




$$
-j \omega \tilde{B}_{z}=-j \omega \mu_{0} \mu_{r_{z z}} \tilde{H}_{z}=\left[\frac{\partial \tilde{E}_{x}}{\partial y}-\frac{\partial \tilde{E}_{y}}{\partial x}\right] .
$$

para modos $\mathrm{TE}_{\mathrm{z}}$, e

$$
\begin{gathered}
-j \omega \tilde{B}_{x}=-j \omega \mu_{0} \mu_{r_{x x}} \tilde{H}_{x}-j \omega \mu_{0} \mu_{r_{x y}} \tilde{H}_{y}=-\frac{\partial \tilde{E}_{z}}{\partial y}, \\
-j \omega \tilde{B}_{y}=-j \omega \mu_{0} \mu_{r y x} \tilde{H}_{x}-j \omega \mu_{0} \mu_{r y y} \tilde{H}_{y}=\frac{\partial \tilde{E}_{z}}{\partial x}, \\
-j \omega \tilde{D}_{z}=-j \omega \varepsilon_{0} \varepsilon_{r_{z z}} \tilde{E}_{z}=\left[\frac{\partial \tilde{H}_{y}}{\partial x}-\frac{\partial \tilde{H}_{x}}{\partial y}\right] .
\end{gathered}
$$

para modos $\mathrm{TM}_{\mathrm{z}}$.

A seguir é apresentado o formalismo com a inclusão dos efeitos de dispersão material.

\section{2-Modelagem de meios dispersivos}

Materiais que apresentam variação dos seus parâmetros eletromagnéticos com a freqüência são classificados como dispersivos. Todos os materiais apresentam algum grau de dispersão, sendo que para alguns meios esse efeito pode ser mais acentuado do que em outros. Uma maneira elegante de se incluir tal efeito nas equações de Maxwell consiste na utilização de modelos materiais tais como os de Debye, Lorentz, ou Drude [32], [34].

Dentre os três modelos materiais citados, somente o de Lorentz e o de Drude são utilizados na modelagem de metamateriais, pois estes possibilitam valores negativos de permissividade elétrica e de permeabilidade magnética. Além disso, o modelo de Drude proporciona uma maior largura de faixa na obtenção de $\varepsilon$ e $\mu$ negativos quando comparados com o modelo de Lorentz [1]. Por estes motivos, esse trabalho adotou o modelo de Drude na análise de meios dispersivos. Este modelo foi originalmente proposto em 1900 por Paul Drude, e visava explicar a condução elétrica dos materiais (especialmente dos metais). Drude aplicou a teoria cinética clássica aos elétrons de um 
material pressupondo que os íons positivos são imóveis. Durante esse movimento, os elétrons colidem com os íons perdendo energia, executando, assim, o movimento de um oscilador harmônico amortecido [34]-[35]. Assim, Drude obteve as seguintes relações para a permissividade elétrica relativa e a permeabilidade magnética relativa:

$$
\begin{aligned}
& \bar{\varepsilon}_{r}(\omega)=1-\frac{\omega_{p}^{2}}{\omega\left(\omega+j \Gamma_{e}\right)}, \\
& \bar{\mu}_{r}(\omega)=1-\frac{\omega_{p}^{2}}{\omega\left(\omega+j \Gamma_{m}\right)} .
\end{aligned}
$$

onde $\omega_{p}$ e $\Gamma_{e, m}$ correspondem à freqüência de plasma (frequiência natural de oscilação do material) e às freqüências de amortecimento, respectivamente. Como exemplo, a Fig. 2.6 apresenta a variação das partes real e imaginária da permissividade elétrica relativa modelada por Drude para diferentes valores da freqüência de plasma.
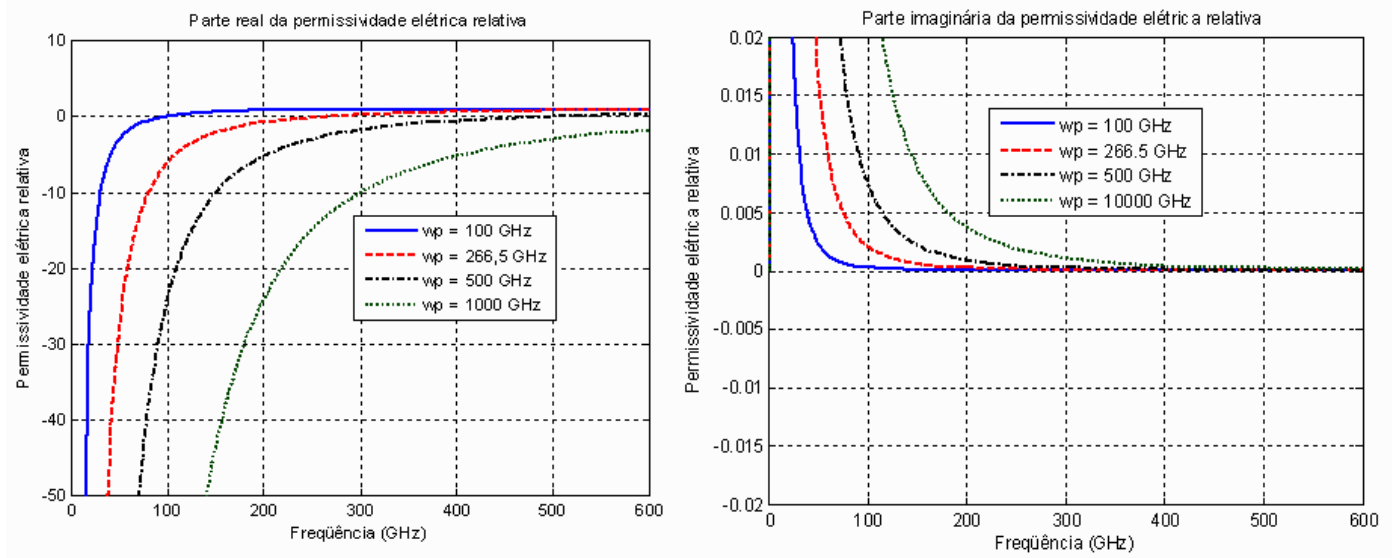

Fig. 2.6 - Permissividade elétrica relativa modelada por Drude. Parte real (esquerda) e parte imaginária (direita), com $\Gamma_{e}=30 \mathrm{MHz}$.

Agora, utilizando-se (2.29) e (2.30) para descrever a permissividade elétrica relativa e a permeabilidade magnética relativa em (2.23)-(2.28), obtém-se:

$$
-j \omega \varepsilon_{0}\left(1-\frac{\omega_{p x}^{2}}{\omega\left(\omega+j \Gamma_{e x}\right)}\right) \tilde{E}_{x}-j \omega \varepsilon_{0}\left(1-\frac{\omega_{p x y}^{2}}{\omega\left(\omega+j \Gamma_{e x y}\right)}\right) \tilde{E}_{y}=\frac{\partial \tilde{H}_{z}}{\partial y}
$$




$$
\begin{gathered}
-j \omega \varepsilon_{0}\left(1-\frac{\omega_{p y x}^{2}}{\omega\left(\omega+j \Gamma_{e y x}\right)}\right) \tilde{E}_{x}-j \omega \varepsilon_{0}\left(1-\frac{\omega_{p y}^{2}}{\omega\left(\omega+j \Gamma_{e y}\right)}\right) \tilde{E}_{y}=-\frac{\partial \tilde{H}_{z}}{\partial x}, \\
-j \omega \mu_{0}\left(1-\frac{\omega_{p z}^{2}}{\omega\left(\omega+j \Gamma_{m z}\right)}\right) \tilde{H}_{z}=\left[\frac{\partial \tilde{E}_{x}}{\partial y}-\frac{\partial \tilde{E}_{y}}{\partial x}\right] .
\end{gathered}
$$

para $\mathrm{TE}_{\mathrm{z}}, \mathrm{e}$

$$
\begin{gathered}
-j \omega \mu_{0}\left(1-\frac{\omega_{p x}^{2}}{\omega\left(\omega+j \Gamma_{m x}\right)}\right) \tilde{H}_{x}-j \omega \mu_{0}\left(1-\frac{\omega_{p x y}^{2}}{\omega\left(\omega+j \Gamma_{m x y}\right)}\right) \tilde{H}_{y}=-\frac{\partial \tilde{E}_{z}}{\partial y}, \\
-j \omega \mu_{0}\left(1-\frac{\omega_{p y x}^{2}}{\omega\left(\omega+j \Gamma_{m y x}\right)}\right) \tilde{H}_{x}-j \omega \mu_{0}\left(1-\frac{\omega_{p y}^{2}}{\omega\left(\omega+j \Gamma_{m y}\right)}\right) \tilde{H}_{y}=\frac{\partial \tilde{E}_{z}}{\partial x}, \\
-j \omega \varepsilon_{0}\left(1-\frac{\omega_{p z}^{2}}{\omega\left(\omega+j \Gamma_{e z}\right)}\right) \tilde{E}_{z}=\left[\frac{\partial \tilde{H}_{y}}{\partial x}-\frac{\partial \tilde{H}_{x}}{\partial y}\right] .
\end{gathered}
$$

para $\mathrm{TM}_{\mathrm{z}}$.

Rearranjando as equações (2.31)-(2.36), tem-se:

$$
\begin{gathered}
-j \omega \varepsilon_{0} \widetilde{E}_{x}+j \omega \frac{\varepsilon_{0} \omega_{p x}^{2} \tilde{E}_{x}}{\omega\left(\omega+j \Gamma_{e x}\right)}-j \omega \varepsilon_{0} \widetilde{E}_{y}+j \omega \frac{\varepsilon_{0} \omega_{p x y}^{2} \tilde{E}_{y}}{\omega\left(\omega+j \Gamma_{e x y}\right)}=\frac{\partial \tilde{H}_{z}}{\partial y}, \\
-j \omega \varepsilon_{0} \widetilde{E}_{x}+j \omega \frac{\varepsilon_{0} \omega_{p y x}^{2} \tilde{E}_{x}}{\omega\left(\omega+j \Gamma_{e y x}\right)}-j \omega \varepsilon_{0} \tilde{E}_{y}+j \omega \frac{\varepsilon_{0} \omega_{p y}^{2} \tilde{E}_{y}}{\omega\left(\omega+j \Gamma_{e y}\right)}=-\frac{\partial \tilde{H}_{z}}{\partial x}, \\
-j \omega \mu_{0} \tilde{H}_{z}+j \omega \frac{\mu_{0} \omega_{p}^{2} \tilde{H}_{z}}{\omega\left(\omega+j \Gamma_{m z}\right)}=\left[\frac{\partial \tilde{E}_{x}}{\partial y}-\frac{\partial \tilde{E}_{y}}{\partial x}\right] .
\end{gathered}
$$

para $\mathrm{TE}_{\mathrm{z}}, \mathrm{e}$

$$
-j \omega \mu_{0} \tilde{H}_{x}+j \omega \frac{\mu_{0} \omega_{p x}^{2} \tilde{H}_{x}}{\omega\left(\omega+j \Gamma_{m x}\right)}-j \omega \mu_{0} \tilde{H}_{y}+j \omega \frac{\mu_{0} \omega_{p x y}^{2} \tilde{H}_{y}}{\omega\left(\omega+j \Gamma_{m x y}\right)}=-\frac{\partial \tilde{E}_{z}}{\partial y}
$$




$$
\begin{gathered}
-j \omega \mu_{0} \tilde{H}_{x}+j \omega \frac{\mu_{0} \omega_{p y x}^{2} \tilde{H}_{x}}{\omega\left(\omega+j \Gamma_{m y x}\right)}-j \omega \mu_{0} \tilde{H}_{y}+j \omega \frac{\mu_{0} \omega_{p y}^{2} \tilde{H}_{y}}{\omega\left(\omega+j \Gamma_{m y}\right)}=\frac{\partial \tilde{E}_{z}}{\partial x} \\
-j \omega \varepsilon_{0} \tilde{E}_{z}+j \omega \frac{\varepsilon_{0} \omega_{p z}^{2} \tilde{E}_{z}}{\omega\left(\omega+j \Gamma_{e z}\right)}=\left[\frac{\partial \tilde{H}_{y}}{\partial x}-\frac{\partial \tilde{H}_{x}}{\partial y}\right] .
\end{gathered}
$$

para $\mathrm{TM}_{\mathrm{z}}$.

Como o meio foi inicialmente considerado livre de fonte, o surgimento da segunda e da quarta parcela no primeiro membro das equações (2.37)-(2.42) acontece devido à propagação de uma onda eletromagnética em um meio dispersivo, originando densidades de corrente magnética e elétrica, com as seguintes expressões, respectivamente:

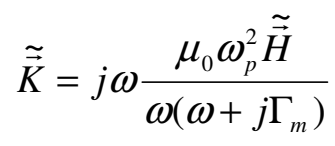

$\mathrm{e}$

$$
\widetilde{\vec{J}}=j \omega \frac{\varepsilon_{0} \omega_{p}^{2} \tilde{\vec{E}}}{\omega\left(\omega+j \Gamma_{e}\right)}
$$

Então, substituindo (2.43) e (2.44) nas equações (2.37)-(2.42), e calculando a transformada inversa de Fourier das equações resultantes, obtém-se:

$$
\begin{gathered}
\varepsilon_{0} \frac{\partial E_{x}}{\partial t}+J_{x}+\varepsilon_{0} \frac{\partial E_{y}}{\partial t}+J_{x y}=\frac{\partial H_{z}}{\partial y}, \\
\varepsilon_{0} \frac{\partial E_{x}}{\partial t}+J_{y x}+\varepsilon_{0} \frac{\partial E_{y}}{\partial t}+J_{y}=-\frac{\partial H_{z}}{\partial x}, \\
\mu_{0} \frac{\partial H_{z}}{\partial t}+K_{z}=\left[\frac{\partial E_{x}}{\partial y}-\frac{\partial E_{y}}{\partial x}\right] .
\end{gathered}
$$

para $\mathrm{TE}_{\mathrm{z}}, \mathrm{e}$ 


$$
\begin{gathered}
\mu_{0} \frac{\partial H_{x}}{\partial t}+K_{x}+\mu_{0} \frac{\partial H_{y}}{\partial t}+K_{x y}=-\frac{\partial E_{z}}{\partial y} \\
\mu_{0} \frac{\partial H_{x}}{\partial t}+K_{y x}+\mu_{0} \frac{\partial H_{y}}{\partial t}+K_{y}=\frac{\partial E_{z}}{\partial x} \\
\varepsilon_{0} \frac{\partial E_{z}}{\partial t}+J_{z}=\left[\frac{\partial H_{y}}{\partial x}-\frac{\partial H_{x}}{\partial y}\right]
\end{gathered}
$$

para $\mathrm{TM}_{\mathrm{z}}$.

Neste trabalho, a solução de (2.45)-(2.50) necessita das equações diferenciais dos vetores densidade de corrente magnética e elétrica, essas equações são obtidas a seguir:

Multiplicando (2.43) e (2.44) por $\omega\left(\omega+j \Gamma_{e, m}\right)$, obtém-se:

$$
\begin{aligned}
& \omega^{2} \tilde{\vec{K}}+j \omega \Gamma_{m} \tilde{\vec{K}}=j \omega \mu_{0} \omega_{p}^{2} \tilde{\vec{H}}, \\
& \omega^{2} \tilde{\vec{J}}+j \omega \Gamma_{e} \tilde{\vec{J}}=j \omega \varepsilon_{0} \omega_{p}^{2} \tilde{\vec{E}} .
\end{aligned}
$$

Calculando a transformada inversa de Fourier de (2.51) e de (2.52), e integrando ambos os membros das equações resultantes uma vez com respeito ao tempo, tem-se:

$$
\begin{gathered}
\omega^{2} \tilde{\vec{A}}+j \omega \Gamma_{m, e} \tilde{\vec{A}}=j g_{0} \omega_{p}^{2} \tilde{\vec{C}} \\
-\frac{\partial^{2} \vec{A}}{\partial t^{2}}-\Gamma_{m, e} \frac{\partial \vec{A}}{\partial t}=-g_{0} \omega_{p}^{2} \frac{\partial \vec{C}}{\partial t}, \\
\int\left(-\frac{\partial^{2} \vec{A}}{\partial t^{2}}-\Gamma_{m, e} \frac{\partial \vec{A}}{\partial t}\right) d t=\int\left(-g_{0} \omega_{p}^{2} \frac{\partial \vec{C}}{\partial t} d t\right) . \\
\frac{\partial \vec{A}}{\partial t}+\Gamma_{m, e} \vec{A}=g_{0} \omega_{p}^{2} \vec{C}
\end{gathered}
$$


onde:

$$
\begin{aligned}
& \vec{A}=\vec{K} \text { ou } \vec{J}, \\
& g=\mu \text { ou } \varepsilon \text { e, } \\
& \vec{C}=\vec{H} \text { ou } \vec{E} .
\end{aligned}
$$

Logo, as equações diferenciais temporais utilizadas neste trabalho para simular a propagação da onda eletromagnética na cobertura de invisibilidade são:

$$
\begin{aligned}
& \mu_{0} \frac{\partial H_{x}}{\partial t}+K_{x}+\mu_{0} \frac{\partial H_{y}}{\partial t}+K_{x y}=-\frac{\partial E_{z}}{\partial y}, \\
& \mu_{0} \frac{\partial H_{x}}{\partial t}+K_{y x}+\mu_{0} \frac{\partial H_{y}}{\partial t}+K_{y}=\frac{\partial E_{z}}{\partial x}, \\
& \frac{\partial K_{x}}{\partial t}+\Gamma_{m x} K_{x}=\mu_{0} \omega_{p x}^{2} H_{x}, \\
& \frac{\partial K_{x y}}{\partial t}+\Gamma_{m x y} K_{x y}=\mu_{0} \omega_{p x y}^{2} H_{y} \\
& \frac{\partial K_{y}}{\partial t}+\Gamma_{m y} K_{y}=\mu_{0} \omega_{p y}^{2} H_{y}, \\
& \frac{\partial K_{y x}}{\partial t}+\Gamma_{m y x} K_{y x}=\mu_{0} \omega_{p y x}^{2} H_{x}, \\
& \varepsilon_{0} \frac{\partial E_{z}}{\partial t}+J_{z}=\left[\frac{\partial H_{y}}{\partial x}-\frac{\partial H_{x}}{\partial y}\right], \\
& \frac{\partial J_{z}}{\partial t}+\Gamma_{e z} J_{z}=\varepsilon_{0} \omega_{p z}^{2} E_{z}
\end{aligned}
$$


Vários métodos numéricos podem ser adotados na solução das equações (2.54)(2.61), tais como os métodos dos momentos (MoM) [36], elementos finitos (FEM) [36], volumes finitos [36], e o método das diferenças finitas no domínio do tempo (FDTD) [37]-[38]. O método FDTD vem ganhando vários adeptos nos últimos anos, principalmente em virtude dos notáveis avanços tecnológicos conseguidos no desenvolvimento de computadores cada vez mais potentes. Isso tem permitido inclusive que estruturas complexas, algo impensável alguns anos atrás para este método, sejam analisadas eficientemente. A versatilidade deste método, aliado à sua implementação mais simples quando comparada aos demais, permite a investigação de fenômenos eletromagnéticos complexos em estruturas baseadas em metamateriais, tais como: índice de refração negativo, índice de refração próximo de zero e plásmons de superfície [15]. Diante dos motivos descritos acima, este método foi escolhido para este trabalho. Os aspectos relevantes do seu formalismo são apresentados na próxima seção.

\section{3-Método das diferenças finitas no domínio do tempo (FDTD)}

O método FDTD foi originalmente proposto por Yee em 1966 [39]. Neste método, tanto os operadores diferenciais espaciais quanto o temporal, presentes nas equações de Maxwell dependentes do tempo, são discretizados em termos de diferenças centradas possibilitando, assim, a obtenção de um esquema com precisão de segunda ordem.

Em seu trabalho, Yee apresentou equações discretas de atualização temporal dispostas no esquema leapfrog [39], onde $\vec{E}$ e $\vec{H}$ são calculados de forma intercalada no tempo, conforme mostrado na Fig. 2.7. Isso implica que as componentes de campo elétrico e magnético não são co-localizadas (não estão em um mesmo ponto na malha). A discretização temporal será definida em termos da variável inteira $i$ e passo $\Delta t$, enquanto que a espacial pelas variáveis $m$ e $n$ (relativas aos eixos $x$ e y) e passos $\Delta x$ e $\Delta y$, respectivamente. 


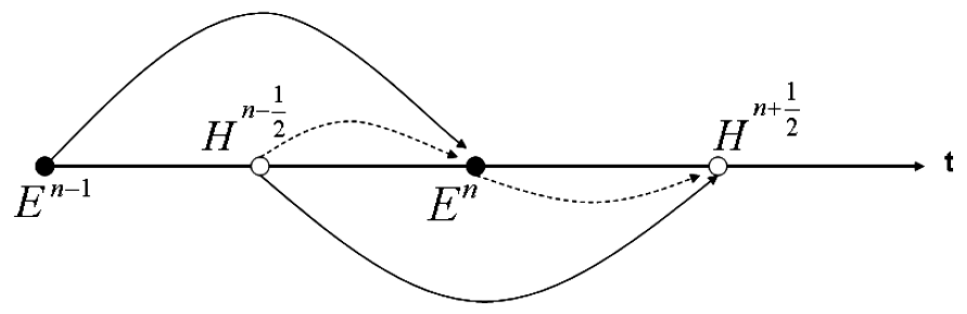

Fig. 2.7 - Esquema leapfrog de evolução temporal do método FDTD.

Portanto, com base nesta figura, o vetor campo elétrico é calculado em um instante de tempo $n$ utilizando as componentes dos vetores de campo elétrico e magnético previamente calculadas. No instante de tempo posterior $(n+1 / 2)$, o vetor campo magnético é calculado utilizando as componentes dos vetores de campo elétrico e magnético do instante de tempo $n$ e $n-1 / 2$, respectivamente. Este processo se repete sucessivamente até que o tempo de propagação desejado seja atingido.

A discretização espacial consiste na aproximação por diferenças finitas das derivadas espaciais contidas em (2.54)-(2.61), sendo que esta discretização depende da configuração da célula adotada no problema. Neste trabalho foi adotada a célula de discretização de Yee bidimensional, conforme mostrada na Fig. 2.8.

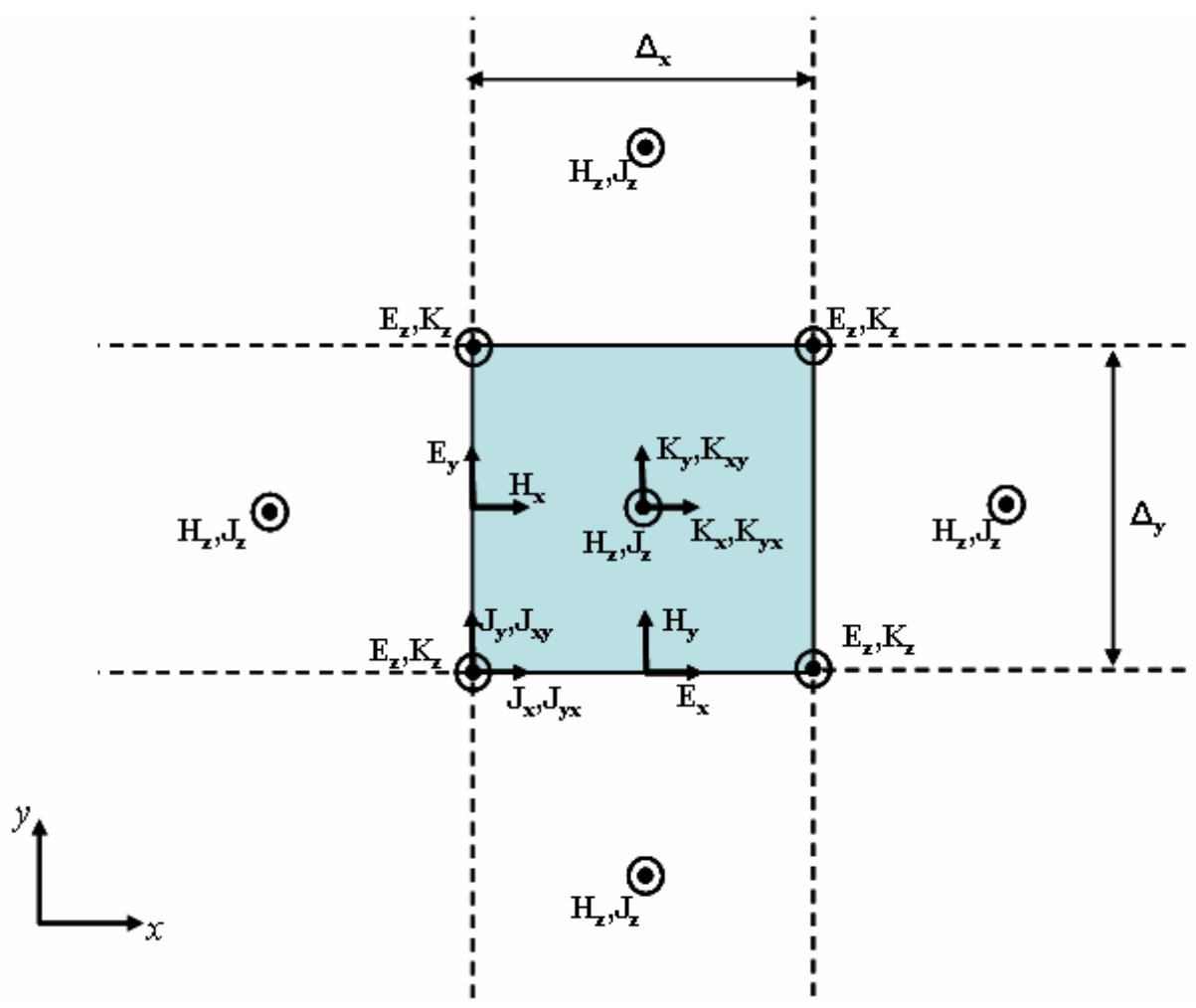

Fig. 2.8 - Célula de discretização espacial do método 2D-FDTD. 
Um aspecto bastante importante na modelagem é a dispersão numérica intrínseca do método FDTD. Para evitar que tal característica degrade a precisão dos resultados, o comprimento do lado das células deve obedecer a um limite superior, o qual é determinado por uma fração do comprimento de onda $\lambda$ (em geral $\lambda / 30$ é adequado para vários tipos de problemas eletromagnéticos) [37]. Além disso, é necessário que o método seja estável, o que requer a adoção de um critério de estabilidade. O critério de Courant [37] é o mais largamente utilizado na caracterização da estabilidade deste método numérico. Este critério impõe um limite no incremento temporal escolhido em função da resolução da célula de discretização do problema analisado. O critério de estabilidade de Courant, para o caso bidimensional, é dado por [37]:

$$
\Delta t \leq \frac{1}{c \sqrt{\frac{1}{(\Delta x)^{2}}+\frac{1}{(\Delta y)^{2}}}}
$$

onde $c$ é a velocidade da luz no espaço livre.

No apêndice B é mostrado um fluxograma de um algoritmo FDTD.

Quando se pretende simular a propagação eletromagnética por uma estrutura de tamanho finito, um bom desempenho do método FDTD está relacionado à implementação de condições de contorno absorventes (ABC - absorbing boundary conditions) [37]-[38] para truncar o tamanho da janela computacional. Este trabalho adotou a técnica $\mathrm{ABC}$ conhecida como condição de camadas perfeitamente casadas (PML - perfectly matched layers) [40]. A descrição desta técnica é apresentada no Apêndice C.

Utilizando a célula de discretização espacial mostrada na Fig. 2.8, as equações (2.54)-(2.61) discretizadas são dada a seguir: 


$$
\begin{aligned}
& H_{x}^{i+1 / 2}(m, n)= \frac{\left[\frac{\mu_{0} \mu_{\infty x}(m, n) A-\mu_{0} \mu_{\infty x}(m, n) B}{\Delta t}-\frac{\mu_{0} \sigma_{y}(m, n)}{2 \varepsilon_{0}}\right]}{\left[\frac{\mu_{0} \mu_{\infty x}(m, n) A-\mu_{0} \mu_{\infty x}(m, n) B}{\Delta t}+\frac{\mu_{0} \sigma_{y}(m, n)}{2 \varepsilon_{0}}\right]} H_{x}^{i-1 / 2}(m, n)- \\
& {\left[A\left[\frac{E_{z}^{i}(m, n+1)-E_{z}^{i}(m, n)}{\Delta y}\right]+B C+A D-B E-B F+A G\right] } \\
& {\left[\frac{\mu_{0} \mu_{\infty x}(m, n) A-\mu_{0} \mu_{\infty x}(m, n) B}{\Delta t}+\frac{\mu_{0} \sigma_{y}(m, n)}{2 \varepsilon_{0}}\right] }
\end{aligned}
$$

$$
\begin{aligned}
H_{y}^{i+1 / 2}(m, n)= & \frac{\left[\frac{\mu_{0} \mu_{\infty y}(m, n) H-\mu_{0} \mu_{\infty y}(m, n) I}{\Delta t}-\frac{\mu_{0} \sigma_{x}(m, n)}{2 \varepsilon_{0}}\right]}{\left[\frac{\mu_{0} \mu_{\infty y}(m, n) H-\mu_{0} \mu_{\infty y}(m, n) I}{\Delta t}+\frac{\mu_{0} \sigma_{x}(m, n)}{2 \varepsilon_{0}}\right]} H_{y}^{i-1 / 2}(m, n)+ \\
& {\left[\frac{\left.H\left[\frac{E_{z}^{i}(m+1, n)-E_{z}^{i}(m, n)}{\Delta x}\right]-I J+H K+I L+I M-H N\right]}{\Delta t}\right] }
\end{aligned}
$$

$$
\begin{aligned}
& K_{x}^{i+1}(m, n)=\frac{1-0,5 \Delta t \Gamma_{m x}(m, n)}{1+0,5 \Delta t \Gamma_{m x}(m, n)} K_{x}^{i}(m, n) \\
& +\frac{\mu_{0} \omega_{p x}^{2}(m, n) \Delta t}{2\left(1-0,5 \Delta t \Gamma_{m x}(m, n)\right)}\left[H_{x}^{i+1 / 2}(m, n)+H_{x}^{i+1 / 2}(m+1, n)\right] \\
& K_{x y}^{i+1}(m, n)=\frac{1-0,5 \Delta t \Gamma_{m x y}(m, n)}{1+0,5 \Delta t \Gamma_{m x y}(m, n)} K_{x y}^{i}(m, n) \\
& +\frac{\mu_{0} \omega_{p x y}^{2}(m, n) \Delta t}{2\left(1-0,5 \Delta t \Gamma_{m x y}(m, n)\right)}\left[H_{y}^{i+1 / 2}(m, n)+H_{y}^{i+1 / 2}(m, n+1)\right] \\
& K_{y}^{i+1}(m, n)=\frac{1-0,5 \Delta t \Gamma_{m y}(m, n)}{1+0,5 \Delta t \Gamma_{m y}(m, n)} K_{y}^{i}(m, n) \\
& +\frac{\mu_{0} \omega_{p y}^{2}(m, n) \Delta t}{2\left(1-0,5 \Delta t \Gamma_{m y}(m, n)\right)}\left[H_{y}^{i+1 / 2}(m, n)+H_{y}^{i+1 / 2}(m, n+1)\right]
\end{aligned}
$$




$$
\begin{aligned}
& K_{y x}^{i+1}(m, n)=\frac{1-0,5 \Delta t \Gamma_{m y x}(m, n)}{1+0,5 \Delta t \Gamma_{m y x}(m, n)} K_{y x}^{i}(m, n) \\
& +\frac{\mu_{0} \omega_{p y x}^{2}(m, n) \Delta t}{2\left(1-0,5 \Delta t \Gamma_{m y x}(m, n)\right)}\left[H_{x}^{i+1 / 2}(m, n)+H_{x}^{i+1 / 2}(m+1, n)\right] \\
& E_{z x}^{i+1}(m, n)=\frac{\left[\frac{\varepsilon_{0} \varepsilon_{\infty z}}{\Delta t}-\frac{\sigma_{x}(m, n)}{2}\right]}{\left[\frac{\varepsilon_{0} \varepsilon_{\infty z}}{\Delta t}+\frac{\sigma_{x}(m, n)}{2}\right]} E_{z x}^{i}(m, n)+\frac{H_{y}(m, n)-H_{y}(m-1, n)}{\Delta x\left[\frac{\varepsilon_{0} \varepsilon_{\infty z}}{\Delta t}-\frac{\sigma_{x}(m, n)}{2}\right]}- \\
& \frac{J_{z x}^{i+1 / 2}(m-1, n)+J_{z x}^{i+1 / 2}(m, n)+J_{z x}^{i+1 / 2}(m-1, n-1)+J_{z x}^{i+1 / 2}(m, n-1)}{4\left[\frac{\varepsilon_{0} \varepsilon_{\infty z}}{\Delta t}-\frac{\sigma_{x}(m, n)}{2}\right]} \\
& \begin{aligned}
E_{z y}^{i+1}(m, n)=\frac{\left[\frac{\varepsilon_{0} \varepsilon_{\infty z}}{\Delta t}-\frac{\sigma_{y}(m, n)}{2}\right]}{\left[\frac{\varepsilon_{0} \varepsilon_{\infty z}}{\Delta t}+\frac{\sigma_{y}(m, n)}{2}\right]} E_{z y}^{i}(m, n)-\frac{H_{x}(m, n)-H_{x}(m, n-1)}{\Delta y\left[\frac{\varepsilon_{0} \varepsilon_{\infty z}}{\Delta t}-\frac{\sigma_{y}(m, n)}{2}\right]}- \\
\frac{J_{z y}^{i+1 / 2}(m-1, n)+J_{z y}^{i+1 / 2}(m, n)+J_{z y}^{i+1 / 2}(m-1, n-1)+J_{z y}^{i+1 / 2}(m, n-1)}{4\left[\frac{\varepsilon_{0} \varepsilon_{\infty z}}{\Delta t}-\frac{\sigma_{y}(m, n)}{2}\right]}
\end{aligned}
\end{aligned}
$$

$$
\begin{aligned}
& J_{z x}^{i+3 / 2}(m, n)=\frac{1-0,5 \Delta t \Gamma_{m y}(m, n)}{1+0,5 \Delta t \Gamma_{m y}(m, n)} J_{z x}^{i+1 / 2}(m, n) \\
& +\frac{\varepsilon_{0} \omega_{p x}^{2}(m, n) \Delta t\left[E_{z x}^{i+1}(m, n)+E_{z x}^{i+1}(m, n+1)+E_{z x}^{i+1}(m+1, n+1)+E_{z x}^{i+1}(m+1, n)\right]}{4\left(1-0,5 \Delta t \Gamma_{m x}(m, n)\right)}
\end{aligned}
$$

$$
\begin{aligned}
& J_{z y}^{i+3 / 2}(m, n)=\frac{1-0,5 \Delta t \Gamma_{m y}(m, n)}{1+0,5 \Delta t \Gamma_{m y}(m, n)} J_{z y}^{i+1 / 2}(m, n) \\
& +\frac{\varepsilon_{0} \omega_{p y}^{2}(m, n) \Delta t\left[E_{z y}^{i+1}(m, n)+E_{z y}^{i+1}(m, n+1)+E_{z y}^{i+1}(m+1, n+1)+E_{z y}^{i+1}(m+1, n)\right]}{4\left(1-0,5 \Delta t \Gamma_{m y}(m, n)\right)}
\end{aligned}
$$


onde:

$$
\begin{aligned}
& A=0,25\left[\begin{array}{l}
\varepsilon_{\infty y}(m, n)+\varepsilon_{\infty y}(m-1, n)+ \\
\varepsilon_{\infty y}(m-1, n+1)+\varepsilon_{\infty y}(m, n+1)
\end{array}\right], \\
& B=0,25\left[\begin{array}{l}
\varepsilon_{r x y}(m, n)+\varepsilon_{r_{x y}}(m-1, n)+ \\
\varepsilon_{r x y}(m-1, n+1)+\varepsilon_{r_{x y}}(m, n+1)
\end{array}\right], \\
& C=\frac{0,25}{\Delta x}\left[\begin{array}{l}
E_{z}^{i}(m+1, n)-E_{z}^{i}(m-1, n)+ \\
E_{z}^{i}(m+1, n+1)-E_{z}^{i}(m-1, n+1)
\end{array}\right], \\
& D=0,5\left[K_{x}^{i}(m, n)+K_{x}^{i}(m-1, n)\right], \\
& E=0,5\left[K_{y}^{i}(m, n)+K_{y}^{i}(m-1, n)\right], \\
& F=0,5\left[K_{y x}^{i}(m, n)+K_{y x}^{i}(m-1, n)\right], \\
& G=0,5\left[K_{x y}^{i}(m, n)+K_{x y}^{i}(m-1, n)\right], \\
& H=0,25\left[\begin{array}{l}
\varepsilon_{\infty x}(m, n)+\varepsilon_{\infty x}(m, n+1)+ \\
\varepsilon_{\infty x}(m-1, n)+\varepsilon_{\infty x}(m-1, n+1)
\end{array}\right], \\
& I=0,25\left[\begin{array}{l}
\varepsilon_{r_{y x}}(m, n)+\varepsilon_{r_{y x}}(m, n+1)+ \\
\varepsilon_{r_{y x}}(m-1, n)+\varepsilon_{r y x}(m-1, n+1)
\end{array}\right], \\
& J=\frac{0,25}{\Delta y}\left[\begin{array}{l}
E_{z}^{i}(m, n+1)-E_{z}^{i}(m, n-1)+ \\
E_{z}^{i}(m+1, n+1)-E_{z}^{i}(m+1, n-1)
\end{array}\right], \\
& K=0,5\left[K_{x}^{i}(m, n)+K_{x}^{i}(m, n-1)\right], \\
& L=0,5\left[K_{y}^{i}(m, n)+K_{y}^{i}(m, n-1)\right],
\end{aligned}
$$


$M=0,5\left[K_{y x}^{i}(m, n)+K_{y x}^{i}(m, n-1)\right]$,

$N=0,5\left[K_{x y}^{i}(m, n)+K_{x y}^{i}(m, n-1)\right]$.

Onde $\varepsilon_{\infty}$ e $\mu_{\infty}$ são, respectivamente, a permissividade relativa e a permeabilidade relativa numa freqüência muito elevada, tendendo ao infinito. A maioria dos artigos existentes na literatura consideram que esses valores são aproximadamente 1. Nesta condição, a equação para esses parâmetros eletromagnéticos se assemelha a (2.29) para a permissividade e a (2.30) para a permeabilidade.

Durante uma iteração temporal, (2.63) e (2.64) são resolvidas, logo em seguida, é a vez das equações dos vetores densidade de corrente magnética, (2.65)-(2.68). Posteriormente, atualiza-se o campo elétrico, (2.69)-(2.70). Por fim, a densidade de corrente elétrica é atualizada, (2.71)-(2.72). Este processo se repete sucessivamente até que o tempo de propagação desejado seja atingido. 


\section{Capítulo 3}

\section{Resultados Numéricos}

\section{Introdução}

Este capítulo apresenta um estudo de validação do formalismo 2D-FDTD proposto neste trabalho para a modelagem de meios dispersivos. O método 2D-FDTD é aplicado na análise de estruturas capazes de manipular a radiação eletromagnética, tendo os resultados sido apresentados no 13th IEEE Conference on Electromagnetic Fields, CEFC, Grécia, Atenas [41]. A abordagem adotada nesta dissertação pode ser igualmente estendida para o caso tridimensional (3D-FDTD).

A seção 3.1 dedica-se a uma breve discussão sobre o desempenho da condição de contorno de camadas perfeitamente casadas (PML - perfectly matched layer), a qual foi empregada na modelagem das bordas do domínio computacional.

Na seção 3.2, são apresentados e discutidos os resultados computados pelo formalismo 2D-FDTD aqui desenvolvido. São investigadas duas estruturas capazes de proporcionar a manipulação da radiação incidente: (a) a cobertura de invisibilidade eletromagnética e (b) o rotacionador de campo. Por meio da verificação na distribuição espacial de campo dessas estruturas, demonstra-se que suas respectivas funcionalidades podem ser asseguradas considerando o caráter dispersivo das mesmas na modelagem computacional. 


\section{1-Condição de contorno absorvente}

Pretendendo evitar erros numéricos provocados por reflexões indesejadas no limite do domínio computacional, adotou-se no presente trabalho, a técnica de paredes perfeitamente casadas PML [40] como condição de contorno absorvente. Os detalhes da PML utilizada nesta dissertação encontram-se no Apêndice C.

A Fig. 3.1 permite uma comparação entre a distribuição espacial da componente $E_{z}$ obtida com e sem a utilização da PML. Os cálculos foram desenvolvidos considerando o espaço livre. A excitação empregada é uma fonte senoidal pontual bidimensional com $30 \mathrm{GHz}$ de freqüência. Na TABELA I são listados os parâmetros relativos à discretização dessas duas simulações. Na ausência da condição de contorno absorvente, a onda é totalmente refletida (Fig. 3.1 (a)). No entanto, quando é utilizada PML (Fig. 3.1 (b)), a onda é satisfatoriamente absorvida.

TABELA I: Parâmetros do domínio computacional empregados no teste de desempenho da PML. $N_{x}$ e $N_{y}$ representam o número de pontos do domínio computacional, $\Delta x$ e $\Delta y$ representam o passo espacial adotado na direção $x$ e $y$, respectivamente. $\Delta t$ é o passo temporal adotado, $\sigma_{\max }$ é a máxima condutividade elétrica utilizada na PML, $d$ é a espessura da PML utilizada e $m$ é o grau do polinômio da PML adotado nessa simulação.

\begin{tabular}{|c|c|c|}
\hline Parâmetros & Fig. 3.1(a) & Fig. 3.1(b) \\
\hline $\boldsymbol{N}_{\boldsymbol{x}} \mathbf{x} \boldsymbol{N}_{\boldsymbol{y}}$ & $250 \times 250$ & $250 \times 250$ \\
\hline $\boldsymbol{\Delta x}(\mathrm{mm})$ & 0,25 & 0,25 \\
\hline $\boldsymbol{\Delta y}(\mathrm{mm})$ & 0,25 & 0,25 \\
\hline $\boldsymbol{\Delta t}(\mathrm{ps})$ & 0,56 & 0,56 \\
\hline $\mathbf{N}^{\mathbf{0}}$ de células de PML & - & 8 \\
\hline $\boldsymbol{\sigma}_{\max }(\mathrm{mm})$ & - & $10 \omega \varepsilon_{0}$ \\
\hline $\boldsymbol{d}$ (polinômio da PML) & - & 2,00 \\
\hline Duração da simulação & - & 2,00 \\
\hline & $200 \boldsymbol{\Delta t}$ & $200 \boldsymbol{t} \boldsymbol{t}$ \\
\hline
\end{tabular}




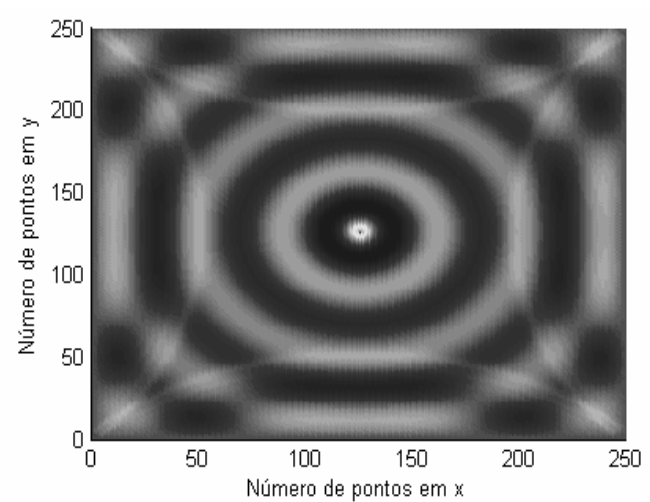

(a)

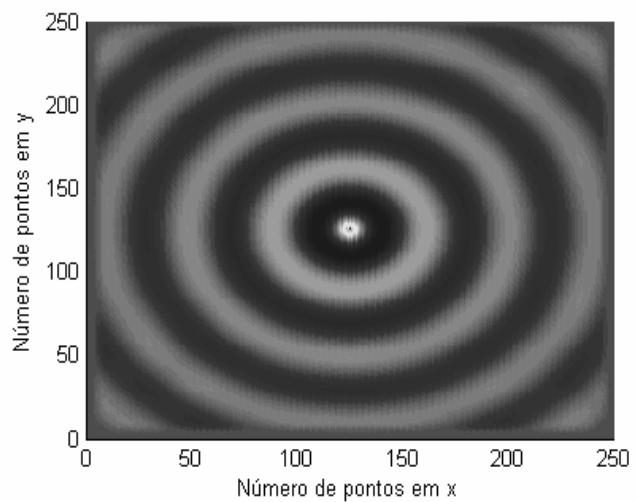

(b)

Fig. 3.1 - Distribuição espacial da componente $E_{z}$ gerada por uma fonte pontual 2D situada no centro do domínio computacional, observada após 200 iterações temporais. (a) Distribuição de campo na ausência de PML e (b) Distribuição de campo utilizando a PML.

Uma maneira de quantificar a eficiência da PML é através do erro relativo de reflexão [37], o qual é definido por:

$$
\text { erro relativo }=\left[\frac{\left|E^{t}-E_{r e f}^{t}\right|}{\max \left|E_{r e f}^{t}\right|}\right] .
$$

Em (3.1), $E^{t}$ representa o campo elétrico obtido na simulação com a PML e $E_{r e f}^{t}$ representa o campo elétrico obtido na simulação sem a PML, considerando uma janela computacional suficientemente grande para que não haja reflexão neste ponto de observação. Para testar a eficiência da PML implementada neste trabalho, foi realizada uma comparação com a PML adotada em [37]. Neste teste, a simulação utilizou um domínio computacional com 100 células na direção $x$ e 100 células na direção $y$. O número de camadas da PML foi fixado em 10 células. A Fig. 3.2 ilustra a visualização esquemática destas especificações. 
A fonte utilizada nesta simulação é descrita pela seguinte expressão:

$$
f(t)=\left\{\begin{array}{ll}
g_{\text {on }} \operatorname{sen}(\omega t) & \text { para } 0 \leq t<b T_{p} \\
\operatorname{sen}(\omega t) & \text { para } b T_{p} \leq t<(b+c) T_{p} \\
g_{\text {off }} \operatorname{sen}(\omega t) & \text { para }(b+c) T_{p} \leq t \leq(b+c+b) T_{p} \\
0 & \text { para }(b+c+b) T_{p}<t
\end{array},\right.
$$

onde:

$T_{p}=1 / f_{0}$ representa o período de um ciclo,

$$
\begin{aligned}
& g_{o n}(t)=10 x_{o n}^{3}-15 x_{o n}^{4}+6 x_{o n}^{5}, \\
& g_{o f f}(t)=1-\left(10 x_{o f f}^{3}-15 x_{o f f}^{4}+6 x_{o f f}^{5}\right), \\
& x_{o n}=1-\left(b T_{p}-t\right) / b T_{p}, \mathrm{e}, \\
& x_{o n}=\left[t+(b+c) T_{p}\right] / b T_{p} .
\end{aligned}
$$

Sendo $b=2$, $c=4$, e $\omega=2 \pi \cdot 30 \times 10^{9} \mathrm{rad} / \mathrm{s}$.

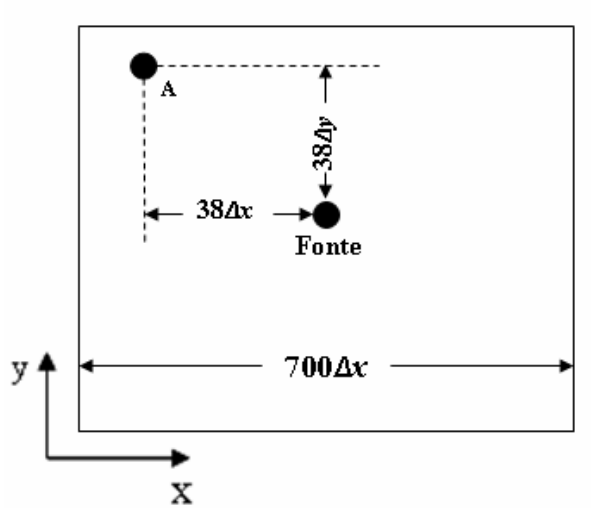

(a)

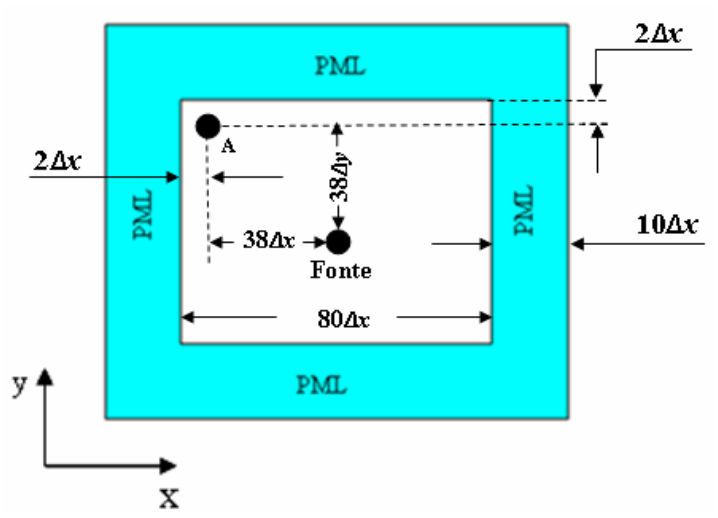

(b)

Fig. 3.2 - Domínio computacional para o cálculo da eficiência da PML. (a) Janela computacional suficientemente grande para obtenção do campo de referência e (b) janela computacional reduzida, envolvida pela PML. O ponto A é o local onde são tomados os campos incidente e refletido. 
A TABELA II lista as especificações utilizadas no teste de eficiência da PML.

TABELA II: Parâmetros do domínio computacional empregados no teste de desempenho da PML. $N_{x}$ e $N_{y}$ representam o número de pontos do domínio computacional, $\Delta x$ e $\Delta y$ representam o passo espacial adotado na direção $x$ e $y$, respectivamente. $\Delta t$ é o passo temporal adotado, $\sigma_{\max }$ é a máxima condutividade elétrica utilizada na PML, $m$ é o grau do polinômio que descreve o perfil de condutividade da PML, $d$ é a espessura da PML utilizada e $m$ é o grau do polinômio da PML adotado na simulação.

\begin{tabular}{|c|c|c|c|c|c|c|}
\hline Parâmetros & \multicolumn{2}{|c|}{ Fig. 3.3(a) } & \multicolumn{2}{c|}{ Fig. 3.3(b) } & \multicolumn{2}{c|}{ Fig. 3.3(c) } \\
\hline & $\begin{array}{c}\text { Utilizado } \\
\text { neste } \\
\text { trabalho }\end{array}$ & $\begin{array}{c}\text { Utilizado em } \\
{[37]}\end{array}$ & $\begin{array}{c}\text { Utilizado } \\
\text { neste } \\
\text { trabalho }\end{array}$ & $\begin{array}{c}\text { Utilizado em } \\
{[37]}\end{array}$ & $\begin{array}{c}\text { Utilizado } \\
\text { neste } \\
\text { trabalho }\end{array}$ & $\begin{array}{c}\text { Utilizado em } \\
{[37]}\end{array}$ \\
\hline $\boldsymbol{N}_{\boldsymbol{x}} \mathbf{X} \boldsymbol{N}_{\mathbf{y}}$ & $100 \times 100$ & $100 \times 100$ & $100 \times 100$ & $100 \times 100$ & $100 \times 100$ & $100 \times 100$ \\
\hline $\boldsymbol{\Delta x}(\mathrm{mm})$ & 0,25 & 0,25 & 0,25 & 0,25 & 0,25 & 0,25 \\
\hline $\boldsymbol{\Delta y}(\mathrm{mm})$ & 0,25 & 0,25 & 0,25 & 0,25 & 0,25 & 0,25 \\
\hline $\boldsymbol{\Delta t}(\mathrm{ps})$ & 0,56 & 0,56 & 0,56 & 0,56 & 0,56 & 0,56 \\
\hline $\begin{array}{c}\mathbf{N}^{\mathbf{0}} \mathbf{d e} \text { células } \\
\text { de PML }\end{array}$ & 10 & 10 & 10 & 10 & 10 & 10 \\
\hline $\boldsymbol{d}(\mathrm{mm})$ & 2,50 & 2,50 & 2,50 & 2,50 & 2,50 & 2,50 \\
\hline $\boldsymbol{m}$ & 2 & 2 & 3 & 3 & 4 & 4 \\
\hline $\boldsymbol{\sigma}_{\mathbf{m a x}}$ & $15 \omega \varepsilon_{0}$ & $\frac{[0,8(m+1)]}{\left(377 \Delta_{x}\right)}$ & $15 \omega \varepsilon_{0}$ & $\frac{[0,8(m+1)]}{\left(377 \Delta_{x}\right)}$ & $15 \omega \varepsilon_{0}$ & $\frac{[0,8(m+1)]}{\left(377 \Delta_{x}\right)}$ \\
\hline $\begin{array}{c}\text { Tempo de } \\
\text { simulação }\end{array}$ & $560 \mathrm{ps}$ & $560 \mathrm{ps}$ & $560 \mathrm{ps}$ & $560 \mathrm{ps}$ & $560 \mathrm{ps}$ & $560 \mathrm{ps}$ \\
\hline
\end{tabular}

Na Fig. 3.3, a comparação da eficiência da PML é apresentada para diferentes valores do grau do polinômio $m$ que descreve o perfil de condutividade da PML (vide Apêndice C). 


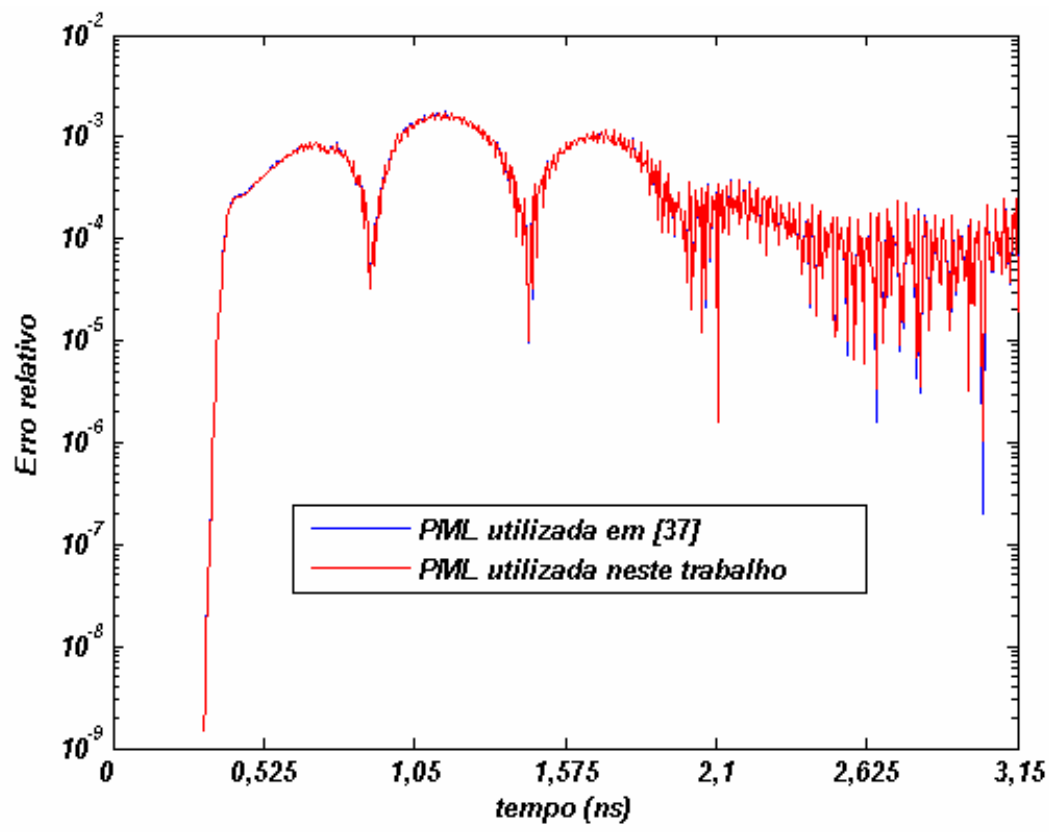

(a)

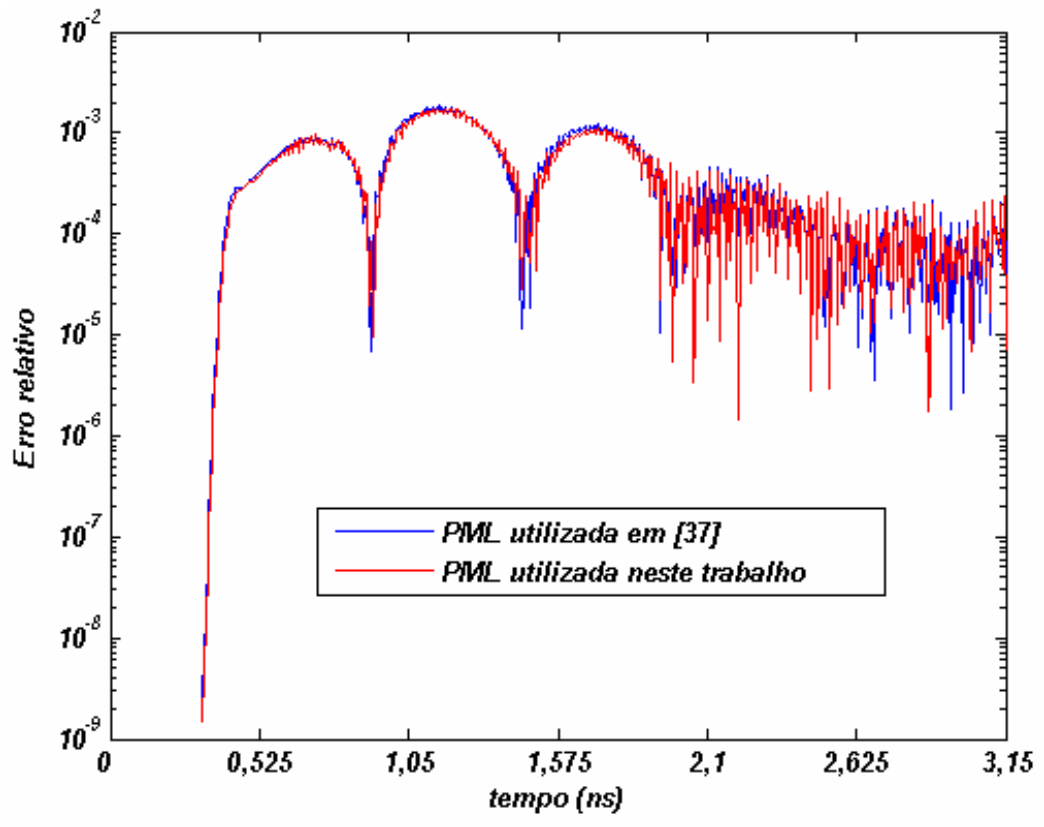

(b) 


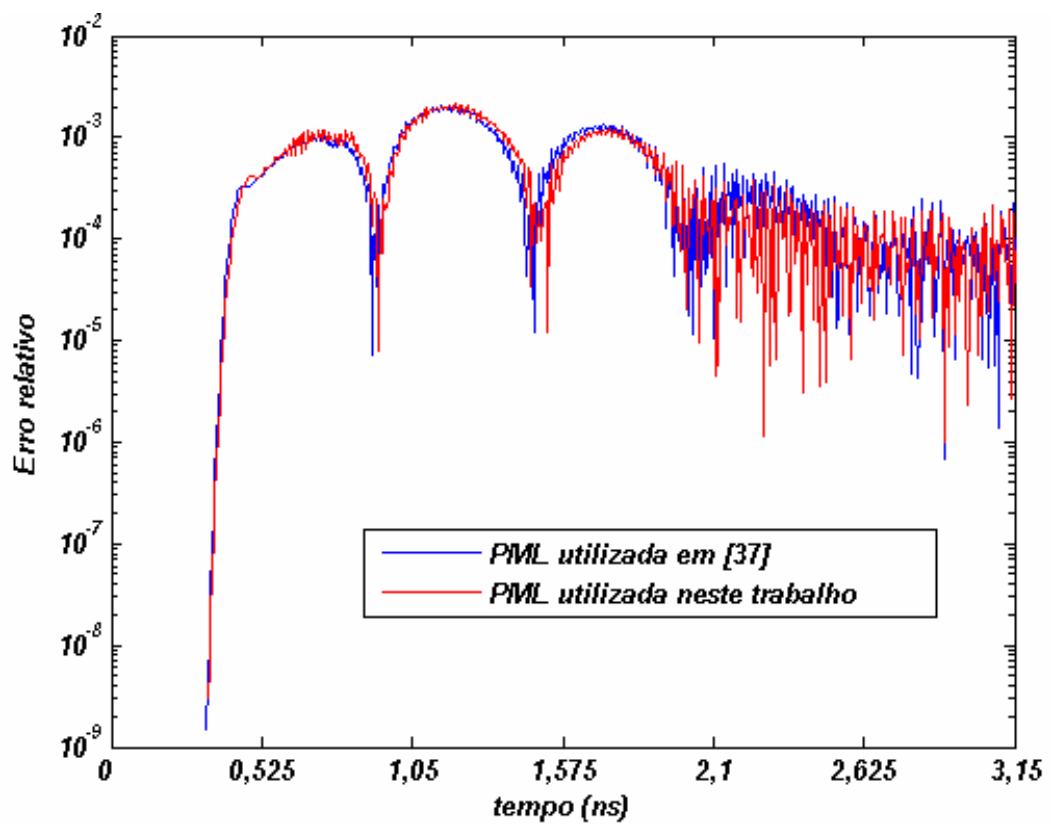

(c)

Fig. 3.3 - Comparação de eficiência de PML utilizada neste trabalho com a de [37] para diferentes valores de $m$, (a) $m=2$, (b) $m=3$ e (c) $m=4$.

Comparando os resultados apresentados na Fig. 3.3, é verificada uma concordância da eficiência da PML adotada neste trabalho com a utilizada em [37]. Deste modo, é assegurado que a PML implementada neste trabalho é robusta o suficiente para ser utilizada na modelagem das estruturas discutidas neste trabalho.

\section{2-Estruturas capazes de proporcionar a manipulação da radiação incidente}

Esta seção é dedicada à análise numérica de estruturas que manipulam a onda eletromagnética. De acordo com o que foi descrito no Capítulo 2, este trabalho adotou o modelo material de Drude para descrever os tensores permissividade elétrica e permeabilidade magnética em função da freqüência. A seguir são analisadas as 
propriedades da cobertura de invisibilidade e do rotacionador de campo eletromagnético considerando o caráter dispersivo dos materiais que os compõem. Vale notar que esta análise ainda é inédita na literatura.

\subsection{1 - Cobertura de invisibilidade eletromagnética}

A cobertura de invisibilidade é uma estrutura que proporciona redução do espalhamento de um determinado objeto. A polarização da onda incidente é suposta como $\mathrm{TM}_{z}$. A fonte utilizada foi uma senóide de frequiência $8,625 \mathrm{GHz}$, a qual foi modelada através da técnica campo total/campo espalhado (TFSF - total field/scattering field).

A Fig. 3.4 apresenta a distribuição espacial de $E_{z}$ para quatro simulações distintas: (a) não considerando o caráter dispersivo da estrutura, (b) considerando o caráter dispersivo da estrutura, (c) considerando o caráter dispersivo, porém, com uma redução dos passos espaciais utilizados em (a) e (b) e (d) idem (c), porém considerando o fator de amortecimento utilizado no metamaterial que constituiu a cobertura de invisibilidade eletromagnética desenvolvida em [18]. Nas três primeiras simulações não foi considerado esse fator de amortecimento.

Os parâmetros do domínio computacional relativos às quatro simulações são listados na TABELA III. Nesta tabela, $N_{x}$ e $N_{y}$ representam o número de pontos do domínio computacional, $\Delta x$ e $\Delta y$ representam o passo espacial adotado na direção $x$ e $y$, respectivamente. $\Delta t$ é o passo temporal adotado, $\sigma_{\max }$ é a máxima condutividade elétrica utilizada na PML, $m$ é o grau do polinômio que descreve o perfil de condutividade da PML, $d$ é a espessura da PML utilizada e $m$ é o grau do polinômio da PML adotado na simulação. O raio do círculo interno dessa estrutura é representado por $a$ e o raio do círculo maior (externo) é representado por $b$. 
TABELA III: Parâmetros do domínio computacional adotados nas quatro simulações distintas da cobertura de invisibilidade eletromagnética (Fig. 3.4): (a) não considerando a dispersão dos materiais, (b) formalismo FDTD considerando o modelo de Drude, (c) considerando Drude e uma redução pela metade dos passos espaciais utilizados em (a) e (b) e (d) idem (c), entretanto considerando o fator de amortecimento do metamaterial utilizado em [18].

\begin{tabular}{|c|c|c|c|c|}
\hline Parâmetros & Fig. 3.4 (a) & Fig. 3.4 (b) & Fig. 3.4 (c) & Fig. 3.4 (d) \\
\hline $\boldsymbol{N}_{\boldsymbol{x}} \mathbf{\times} \boldsymbol{N}_{\boldsymbol{y}}$ & $1200 \times 1200$ & $1200 \times 1200$ & $2400 \times 2400$ & $2200 \times 2200$ \\
\hline$\Delta \boldsymbol{x}(\mathrm{mm})$ & 0,1 & 0,1 & 0,05 & 0,05 \\
\hline$\Delta \boldsymbol{y}(\mathrm{mm})$ & 0,1 & 0,1 & 0,05 & 0,05 \\
\hline $\boldsymbol{\Delta t}(\mathrm{ps})$ & 0,056 & 0,112 & 0,112 & 0,112 \\
\hline $\boldsymbol{a}(\mathrm{mm})$ & 27,00 & 27,00 & 27,00 & 27,00 \\
\hline $\boldsymbol{b}(\mathrm{mm})$ & 59,00 & 59,00 & 59,00 & 59,00 \\
\hline $\begin{array}{c}\mathbf{N}^{\mathbf{o}} \text { de células } \\
\text { de PML }\end{array}$ & 10 & 10 & 20 & 20 \\
\hline $\boldsymbol{\sigma}_{\max }$ & $15 \omega \varepsilon_{0}$ & $15 \omega \varepsilon_{0}$ & $15 \omega \varepsilon_{0}$ & $15 \omega \varepsilon_{0}$ \\
\hline $\boldsymbol{d}(\mathrm{mm})$ & 1,00 & 1,00 & 1,00 & 1,00 \\
\hline $\boldsymbol{m}$ & 2,00 & 2,00 & 2,00 & 2,00 \\
\hline $\begin{array}{c}\text { Duração da } \\
\text { simulação }\end{array}$ & $26000 \Delta \boldsymbol{t}$ & $14000 \Delta \boldsymbol{t}$ & $35000 \Delta \boldsymbol{t}$ & $30000 \Delta \boldsymbol{t}$ \\
\hline
\end{tabular}

Como pode ser visto na Fig. 3.4 (a), o padrão de onda plana inicial quase não é observado na saída da estrutura. Uma justificativa para essa distribuição de campo apresentando uma considerável perturbação deve-se à utilização do método FDTD sem considerar o modelo de Drude. Isto porque em uma região de interface apresentando de um lado valores positivos de $\varepsilon$ ou $\mu$, e de outros valores negativos desses parâmetros, produz uma instabilidade no método FDTD. Esta instabilidade surge tendo em vista que o valor médio de $\varepsilon$ ou $\mu$ será nulo nesse caso, resultando em uma divisão por zero nas equações de discretização utilizadas pelo algoritmo [42]-[43]. A variação pode ser verificada na Fig. 3.5, está que apresenta a distribuição espacial de $\mu_{x x}$.

Na Fig. 3.4 (b), o modelo de Drude é empregado no algoritmo FDTD. Como era esperado, a adoção deste modelo possibilitou reduzir a instabilidade através dos termos adicionados (vetores densidade de corrente magnética e elétrica) ao formalismo FDTD [41]-[42], conforme foi descrito na Seção 2.2 do Capítulo 2. Entretanto, próximo ao círculo menor, ainda pode ser observado uma ligeira elevação de campo elétrico, resultado de variações abruptas dos parâmetros eletromagnéticos naquela região. Neste caso, a variação de campo elétrico é tão acentuada que a discretização espacial padrão 
não satisfaz os critérios de estabilidade do método FDTD [37]-[38]. O diagrama da Fig. 3.4 (b) corresponde a uma discretização espacial da ordem de $\lambda / 350$. Na Fig. 3.4 (c), o passo espacial foi diminuído para $\lambda / 700$. Com esta redução, foi possível eliminar a instabilidade numérica.

Na Fig. 3.4 (c), enquanto a onda se propaga pela estrutura, é observado um atraso na frente de onda que penetra no metamaterial comparada com a parte que se propaga pelo espaço-livre. Assim, há uma compressão da frente de onda na estrutura, até a propagação alcançar o círculo de raio $a$. Neste instante, essa frente é separada e contorna o círculo menor, não havendo propagação da onda na região interna do círculo de raio $a$. Ao contornar esse círculo, é verificada novamente uma compressão da frente de onda plana no metamaterial. Entretanto, desta vez há um adiantamento na frente de onda situada na estrutura com relação à parte que se propaga no espaço-livre. Este comportamento está de acordo com o observado em [18]. Na Fig. 3.4(d), é mostrada a distribuição espacial de $E_{z}$ considerando um fator de amortecimento de $0,01 \mathrm{GHz}$, que corresponde ao valor experimental medido para esses metamateriais em [18]. Este resultado será comparado mais adiante com o resultado experimental obtido em [18].

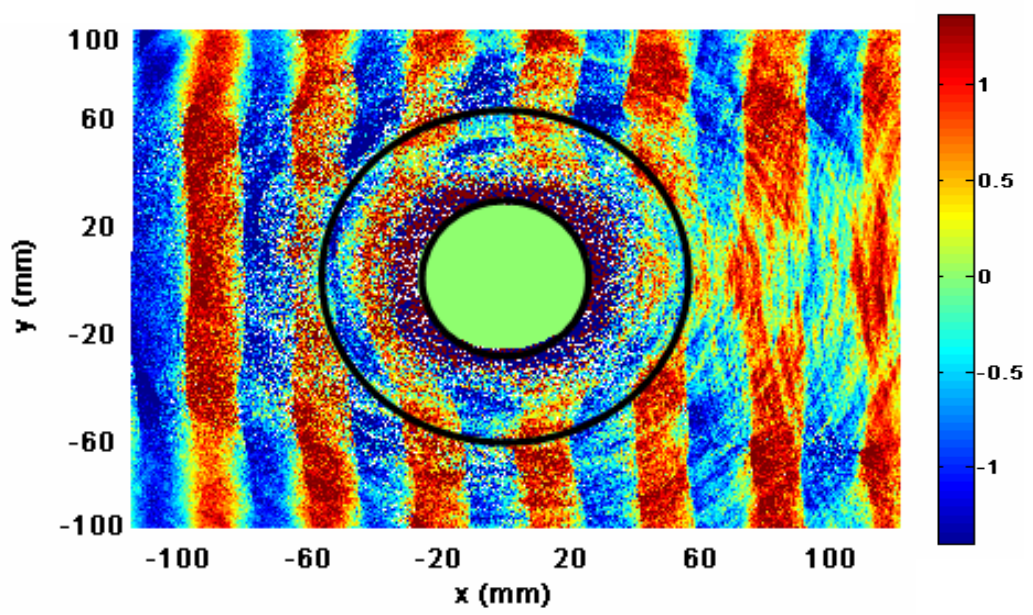

(a) 


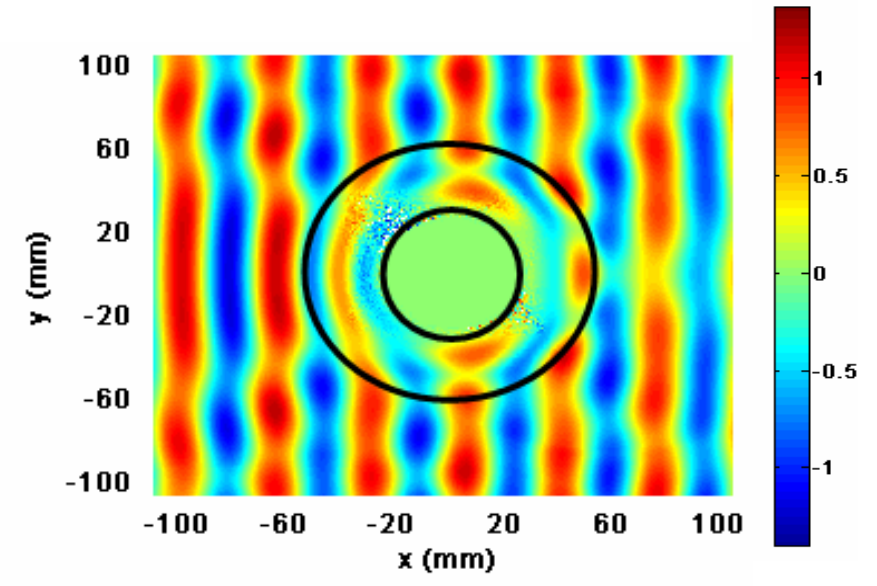

(b)

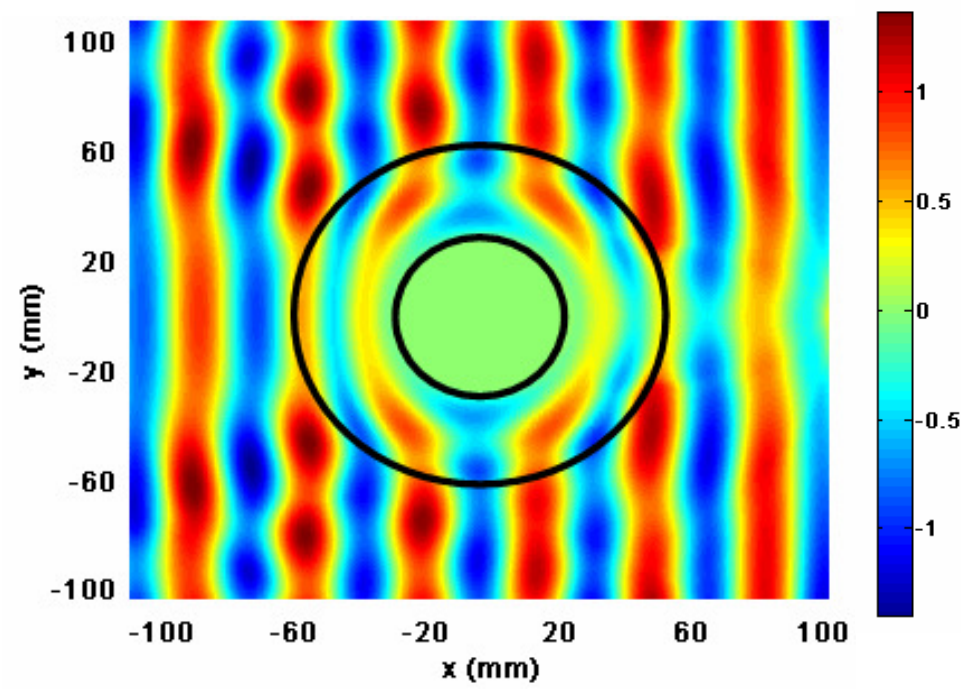

(c) 


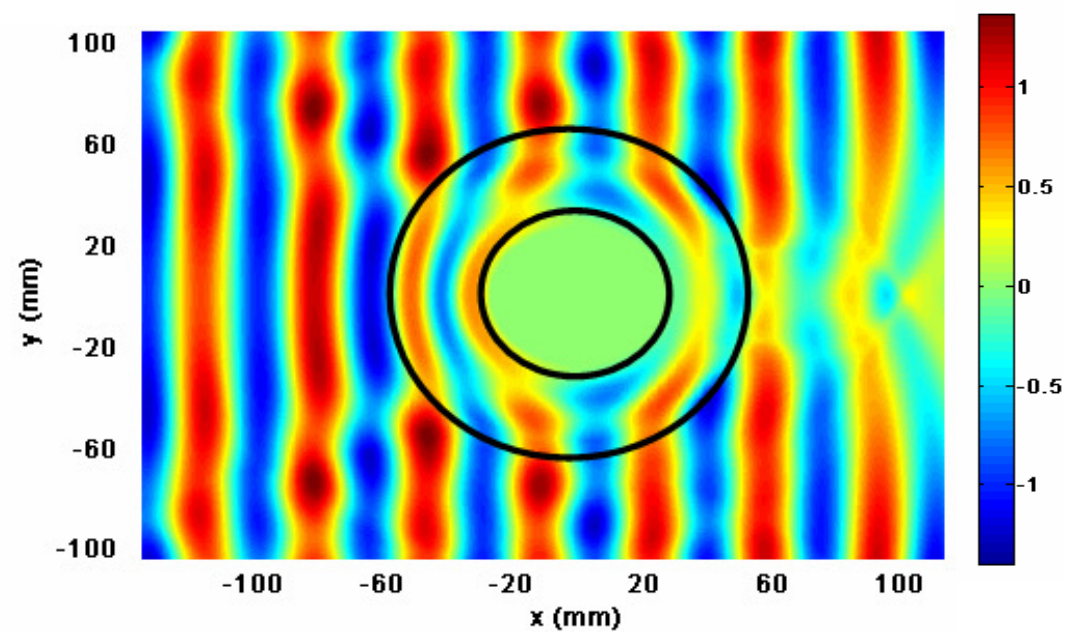

(d)

Fig. 3.4 - Distribuição espacial de $E_{z}$, (a) não considerando a dispersão dos materiais, (b) modelo de Drude incluso no formalismo do método FDTD, (c) idem (b) com passo espacial reduzido à metade do valor adotado em (a) e (b) e (d) idem (c), porém considerando na simulação a perda do material utilizado para fabricação da primeira cobertura de invisibilidade eletromagnética.

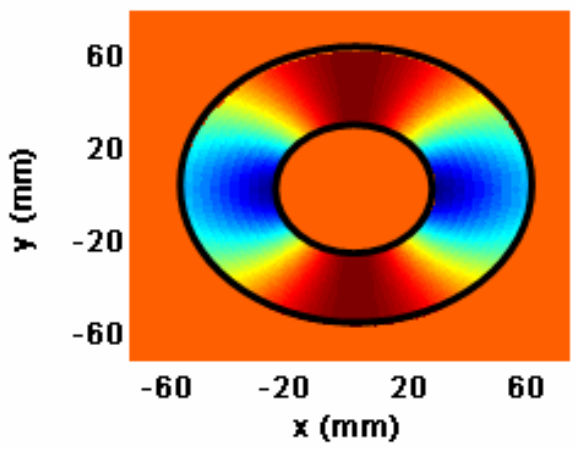

Fig. 3.5 - Distribuição espacial da componente $\mu_{x x}$.

Para validar o método desenvolvido neste trabalho, compararam-se os resultados aqui obtidos com os disponíveis na literatura. Para tal comparação, a Fig.3.6, extraída de [18], apresenta a distribuição espacial da componente de campo elétrico na direção $z$ para dois casos diferentes: (a) estrutura simulada sem considerar a perda do metamaterial e utilizando o método dos elementos finitos e, (b) verificação experimental da primeira cobertura de invisibilidade eletromagnética desenvolvida para a faixa de microondas. 


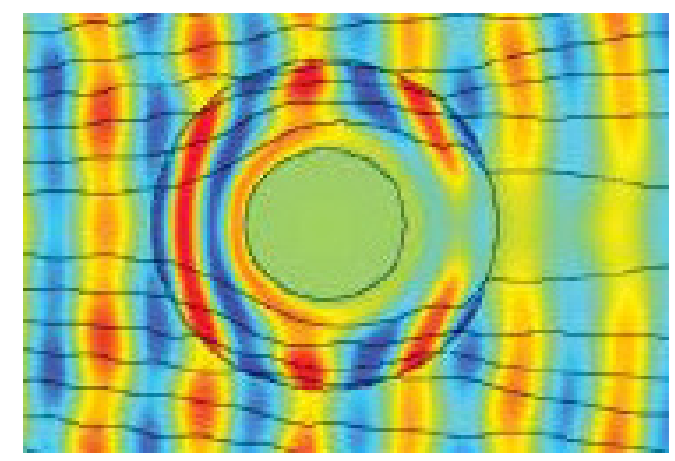

(a)

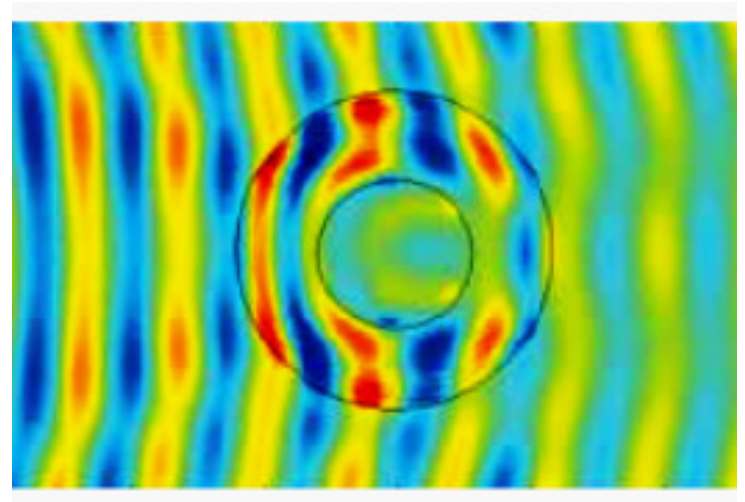

(b)

Fig. 3.6 - Distribuição espacial de $E_{z}$ : (a) simulado, (b) experimental. Resultados extraídos de [18].

Comparando a distribuição espacial para a componente $E_{z}$ mostrada na Fig. 3.4(c), obtida com o presente formalismo, com a distribuição na Fig. 3.6(a) para a mesma componente, simulado com o pacote comercial $\mathrm{COMSOL}^{\circledR}$ baseado em elementos finitos, verifica-se uma grande similaridade entre ambas. Tanto na distribuição espacial da componente $E_{z}$ mostrada na Fig.3.4(d), simulada com o presente formalismo e considerando o fator de amortecimento da cobertura de invisibilidade projetada em [18], quanto na distribuição espacial da mesma componente, apresentada na Fig.3.6(b), obtida experimentalmente, é verificada uma diminuição na amplitude da onda eletromagnética após a passagem pelo dispositivo, causada pela perda do metamaterial. Além disso, é observado na Fig.3.4(d), que não há penetração da radiação no círculo de raio $a$, enquanto que na Fig.3.6(b), verifica-se que a onda eletromagnética penetrou parcialmente nessa região. Essa diferença de distribuição da componente $E_{z}$ se justifica porque a cobertura de invisibilidade eletromagnética analisada experimentalmente em 
[18], foi composta por 10 camadas de metamateriais, enquanto que o resultado mostrado na Fig.3.4(d) considerou uma estrutura com parâmetros eletromagnéticos contínuos e fator de amortecimento do dispositivo analisado em [18]. Através da comparação destas figuras, verifica-se a concordância dos resultados aqui obtidos com os disponíveis na literatura, validando o formalismo desenvolvido neste trabalho.

Durante as investigações da cobertura de invisibilidade, por várias vezes as simulações esbarraram no problema de instabilidade numérica, tal problema só foi solucionado recentemente. No entanto, esse contratempo acabou inviabilizando uma análise mais detalhada nessas estruturas.

\subsection{2 - Rotacionador de campo eletromagnético}

Uma outra estrutura investigada neste trabalho foi o rotacionador de campo eletromagnético. Este dispositivo, como o próprio nome sugere, tem por finalidade produzir uma determinada rotação do campo eletromagnético à medida que este se

propaga pela estrutura. Neste caso, diferente do que ocorre com a cobertura de invisibilidade, não há uma região onde a radiação eletromagnética incidente não pode penetrar. A rotação da frente de onda obedece às seguintes relações (ver Fig. 3.7):

$$
\begin{gathered}
r^{\prime}>=b \therefore\left\{\begin{array}{l}
r^{\prime}=r \\
\theta^{\prime}=\theta, \\
z^{\prime}=z
\end{array}\right. \\
a<r^{\prime}<b \therefore\left\{\begin{array}{l}
r^{\prime}=r, \\
\theta^{\prime}=\theta+\theta_{0} \frac{b-r}{b-a}, \\
z^{\prime}=z
\end{array}\right. \\
r^{\prime} \leq a \therefore\left\{\begin{array}{l}
r^{\prime}=r, \\
\theta^{\prime}=\theta+\theta_{0} . \\
z^{\prime}=z
\end{array}\right.
\end{gathered}
$$


Observe que apenas a coordenada $\theta$ é modificada neste caso. Assim, não há rotação de campo a partir da interface $r=b$ (equação 3.3). As frentes de ondas planas, ao penetrarem no meio metamaterial, serão gradualmente rotacionadas a partir de $0^{\circ}$ na interface $r=b$ até um determinado ângulo $\theta_{0}$ na interface $r=a$ (equação 3.4). A partir de então, elas se propagaram na região interna do círculo de raio menor, composta por espaço-livre. Entretanto, como nesta região a frente de onda já sofreu a rotação desejada, esta deverá permanecer rotacionada de $\theta_{0}$ (equação 3.5). Tendo em vista a facilidade que os meios metamateriais apresentam quanto à alteração de seus parâmetros constitutivos [18]-[21], o rotacionador de campo eletromagnético pode ser implementado a partir de metamateriais [28]. Seus parâmetros constitutivos, obtidos após a transformação de coordenadas, são apresentados a seguir [28]:

$$
\varepsilon_{r}^{\prime}=\mu_{r}^{\prime}=\left[\begin{array}{lll}
c_{11} & c_{12} & c_{13} \\
c_{21} & c_{22} & c_{23} \\
c_{31} & c_{32} & c_{33}
\end{array}\right]
$$

Onde:

$$
\begin{gathered}
c_{11}=1+2 t \cos \theta \sin \theta+t^{2} \sin \theta, \\
c_{12}=c_{21}=-t^{2} \cos \theta \sin \theta-t\left(\cos ^{2} \theta-\sin ^{2} \theta\right), \\
c_{22}=1-2 t \cos \theta \sin \theta+t^{2} \cos \theta, \\
c_{23}=c_{32}=0, \\
c_{33}=1
\end{gathered}
$$

e

$$
t=\frac{\theta_{0} r}{b-a}
$$

Para exemplificar o que foi descrito acima, a Fig. 3.7(a) apresenta a distribuição espacial para a componente $H_{z}$ ao penetrar na região cilíndrica rotacionadora, a distribuição espacial para a mesma componente é mostrada na Fig.3.7(b), com número de iterações no tempo superior ao da Fig.3.7(a). Estas simulações foram obtidas para um rotacionador de campo com raio menor $a=0,25 \mathrm{~m}$, raio maior $b=0,5 \mathrm{~m}$, e $\theta_{0}=90^{\circ}$. A excitação utilizada nesta simulação foi modelada pela técnica TFSF e corresponde a uma senóide de $1,00 \mathrm{GHz}$ de freqüência e polarização $\mathrm{TE}_{z}$. Nestas simulações não foi considerado o caráter dispersivo dos materiais, deste modo, caso ocorra uma mudança 
na freqüência da fonte de excitação, a onda será igualmente rotacionada por este dispositivo, ou seja, a estruturas se comporta como um rotacionador ideal. A distribuição espacial da componente $H_{z}$ para uma fonte com frequiência de $0,90 \mathrm{GHz}$ é apresentada na Fig.3.7(c). Nessa simulação foram considerados os mesmo parâmetros do domínio computacional dos dois casos anteriormente investigados, exceto que essa simulação utilizou uma fonte de freqüência igual a $0,90 \mathrm{GHz}$. Conforme observado nas Fig.3.7(b) e Fig.3.7(c), o dispositivo rotaciona de modo similar tanto a onda incidente com freqüência de $1,00 \mathrm{GHz}$ quanto a radiação produzida por uma fonte senoidal com freqüência de $0,90 \mathrm{GHz}$.

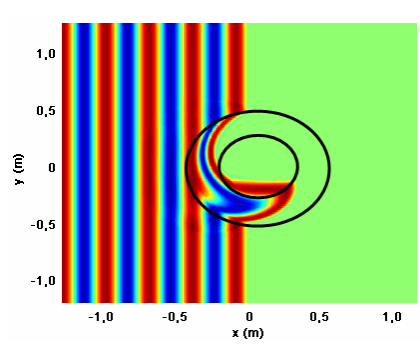

(a)

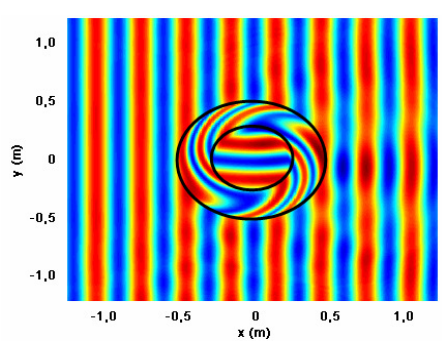

(b)

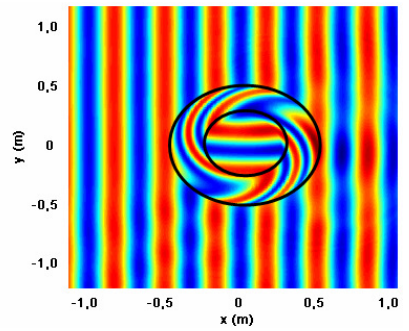

(c)

Fig. 3.7 - Distribuição espacial da componente $H_{z}$ (a)após penetrar no cilindro de metamaterial,

(b) completa simulação com frequiência da fonte de excitação igual a 1,00GHz e (c) idem (b) com freqüência igual a $0,90 \mathrm{GHz}$.

A seguir são apresentados os resultados para um rotacionador de campo com a inclusão do modelo de Drude. Por se tratar de uma estrutura baseada em metamateriais, é de fundamental importância que a natureza dispersiva destes meios seja também levada em consideração. Todos os trabalhos relacionados a este dispositivo disponíveis na literatura não levam em consideração esta característica. Assim, os próximos exemplos apresentam, como no caso da cobertura de invisibilidade, uma contribuição inédita deste trabalho. Sendo assim, a Fig.3.8 apresenta a distribuição de campo magnético para a componente $H_{z}$ obtida com formalismo 2D-FDTD desenvolvido neste trabalho. Foram consideradas três freqüências diferentes para a fonte de excitação (também modelada como TFSF): (a) 1,00GHz (freqüência para a qual o rotacionador foi projetado), (b) $0,90 \mathrm{GHz}$ e (c) $1,02 \mathrm{GHz}$. O objetivo é verificar como variações nos parâmetros de projeto do dispositivo (a freqüência de excitação, neste caso) influenciam o desempenho da estrutura. Os parâmetros do domínio computacional relacionados às três simulações são listados na TABELA IV. 
TABELA IV: Parâmetros do domínio computacional adotados na simulação do rotacionador de campo eletromagnético. $N_{x}$ e $N_{y}$ representam o número de pontos do domínio computacional, $\Delta x$ e $\Delta y$ representam o passo espacial adotado na direção $x$ e $y$, respectivamente. $\Delta t$ é o passo temporal adotado, $\sigma_{\max }$ é a máxima condutividade elétrica utilizada na PML, $d$ é a espessura da PML utilizada e $m$ é o grau do polinômio da PML adotado na simulação.

\begin{tabular}{|c|c|c|c|}
\hline Parâmetros & Fig. 3.7(a) & Fig. 3.7 (b) & Fig. $3.7(\mathrm{c})$ \\
\hline$N_{x} \times N_{y}$ & $1200 \times 1200$ & $1200 \times 1200$ & $1200 \times 1200$ \\
\hline$\Delta \boldsymbol{x}(\mathrm{mm})$ & 2,5 & 2,5 & 2,5 \\
\hline$\Delta y(\mathrm{~mm})$ & 2,5 & 2,5 & 2,5 \\
\hline$\Delta t(\mathrm{ps})$ & 2,8 & 2,8 & 2,8 \\
\hline $\boldsymbol{a}(\mathrm{m})$ & 0,25 & 0,25 & 0,25 \\
\hline $\boldsymbol{b}(\mathrm{m})$ & 0,5 & 0,5 & 0,5 \\
\hline $\mathrm{N}^{\circ}$ de células de $\mathrm{PML}$ & 10 & 10 & 10 \\
\hline$\sigma_{\max }$ & $15 \omega \varepsilon_{0}$ & $15 \omega \varepsilon_{0}$ & $15 \omega \varepsilon_{0}$ \\
\hline $\boldsymbol{d}(\mathrm{mm})$ & 25 & 25 & 25 \\
\hline$m$ & 2,00 & 2,00 & 2,00 \\
\hline Duração da simulação & $14000 \Delta t$ & $14000 \Delta t$ & $14000 \Delta t$ \\
\hline
\end{tabular}

A distribuição espacial da componente $H_{z}$ supondo uma freqüência de excitação de $1,02 \mathrm{GHz}$, ou seja, uma variação de $2 \%$ na freqüência na qual o dispositivo foi projetado, é mostrada na Fig.3.8(b). Em seguida, foi simulada a propagação da onda nesse dispositivo, supondo uma frequiência de $0,90 \mathrm{GHz}$, ou seja, uma variação de $10 \%$ na freqüência na qual o rotacionador foi projetado. A distribuição espacial da componente $H_{z}$ para essa simulação é mostrada na Fig. 3.8 (c). A comparação entre esses resultados evidencia que a operação do rotacionador é significativamente sensível a desvios da frequiência de radiação incidente em relação à frequiência para a qual a estrutura foi projetada. Adicionalmente, verificou-se a importância em considerar a dispersão dos materiais durante as simulações, pois a verificação da sensibilidade da resposta eletromagnética do rotacionador com relação à freqüência da onda incidente, foi realizada através da inclusão do modelo de Drude no formalismo. 


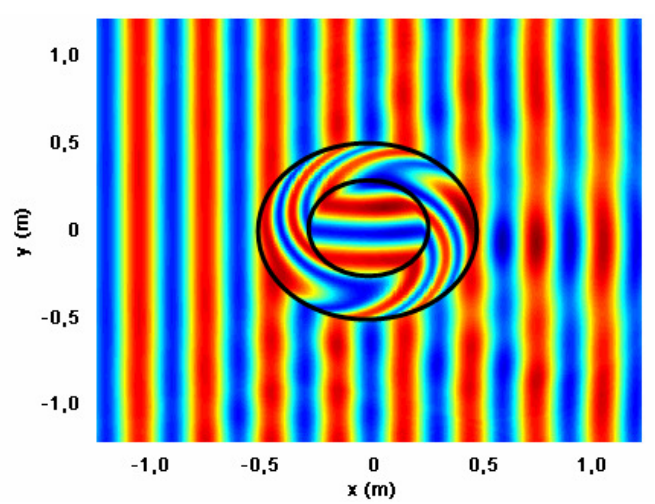

(a)

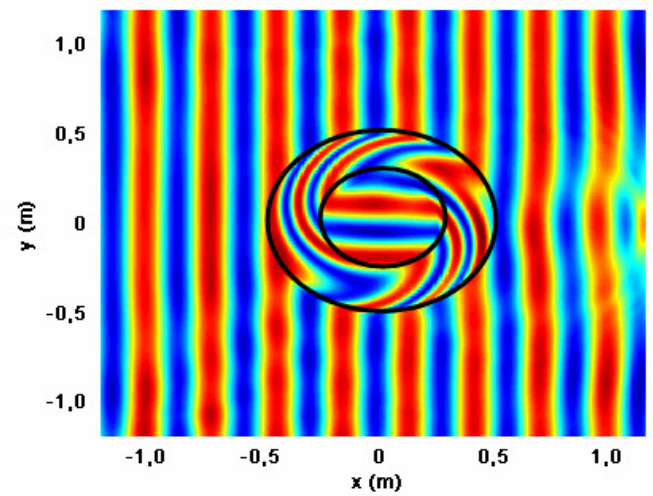

(b)

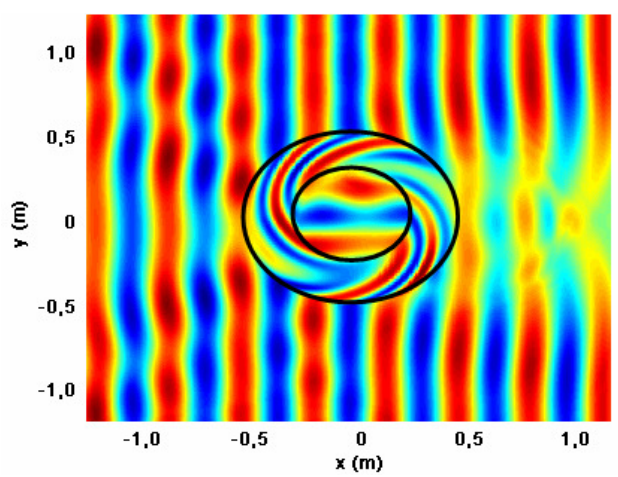

(c)

Fig. 3.8 - Distribuição espacial de $H_{z}$, (a) freqüência da fonte de excitação de 1,00 GHz, (b) freqüência de 1,02GHz e (c) freqüência de $0,90 \mathrm{GHz}$.

Para avaliar o grau de espalhamento das frentes de ondas à medida que a freqüência da radiação incidente desvia-se de $1,00 \mathrm{GHz}$ (freqüência para a qual o rotacionador foi projetado), foi calculado o espalhamento da seção reta (scattering cross section - SCS) no intervalo de $0,80 \mathrm{GHz}$ a $1,20 \mathrm{GHz}$. Os valores de SCS calculados estão normalizados pela frequiência de operação do dispositivo. O gráfico SCS normalizado em função da freqüência é mostrado na Fig. 3.9. 


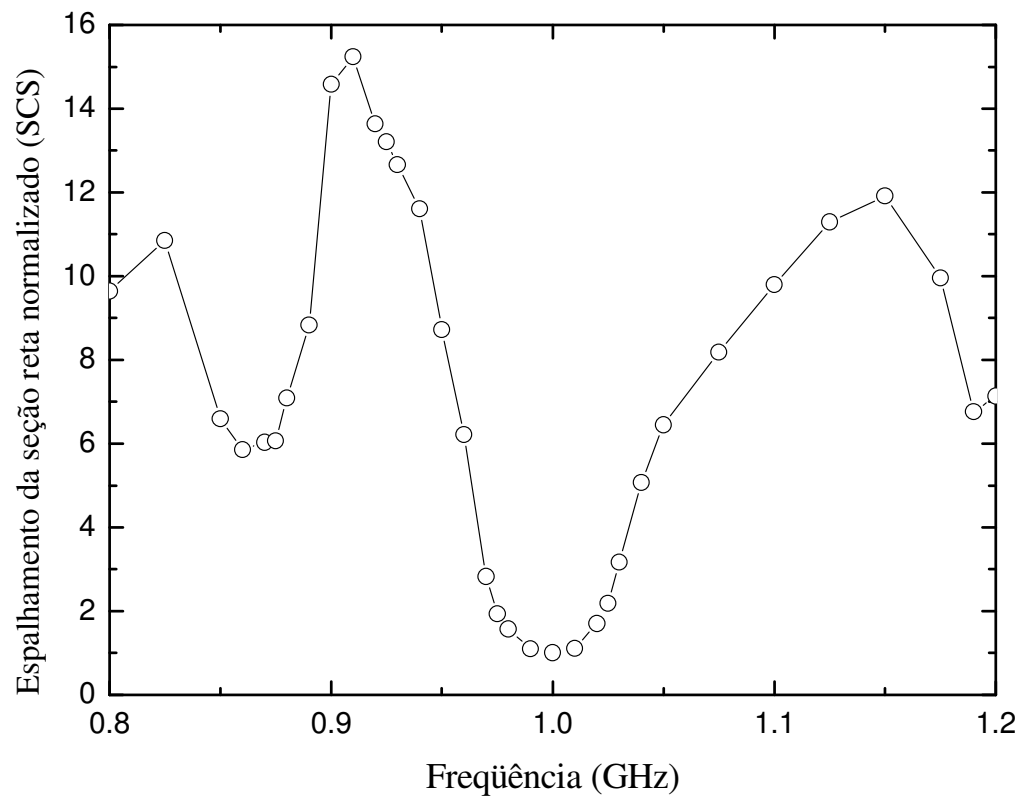

Fig. 3.9 - Espalhamento da seção reta normalizada (SCS) vs frequiência para o rotacionador de campo analisado neste trabalho.

$\mathrm{Na}$ freqüência de $0,96 \mathrm{GHz}$, o valor do SCS é 15 vezes o menor valor do SCS calculado. Conforme esperado, o menor valor do SCS é obtido para a freqüência na qual o rotacionador foi projetado, ou seja, 1,00GHz. Na faixa que vai de $0,97 \mathrm{GHz}$ a $1,02 \mathrm{GHz}$, os valores de SCS não superam em 3 vezes o valor do SCS para a freqüência de 1,00GHz. Para essa faixa de freqüência, a distribuição espacial da componente $H_{z}$ possivelmente não é afetada, conforme mostrado na Fig.3.8(b). Logo, fora dessa faixa de frequiência, a distribuição espacial dessa mesma componente pode apresentar perturbações, semelhante à distribuição mostrada na Fig.3.8(c). 


\section{Conclusões}

Este trabalho apresentou a extensão do formalismo bi-dimensional baseado em diferenças finitas no domínio do tempo (2D-FDTD) para simular a propagação de ondas eletromagnéticas em estruturas capazes de proporcionar a manipulação da radiação incidente, considerando o caráter dispersivo destes meios.

Primeiramente, foi mostrado o procedimento de cálculo dos tensores permissividade elétrica e permeabilidade magnética, o que foi realizado empregando transformação de coordenadas espaciais. Desde modo, foi possível descrever matematicamente a dependência radial dos elementos tensoriais. Sabendo que todo material é dispersivo, e que o efeito desta característica pode ser mais acentuado em alguns meios do que em outros, este trabalho incluiu no formalismo a dispersão dos materiais através do modelo de Drude.

Posteriormente, a modelagem numérica desenvolvida foi utilizada na investigação das seguintes estruturas: (a) a cobertura de invisibilidade e (b) o rotacionador de campo. A modelagem dessas estruturas, levando-se em conta a dispersão material, mostrou não somente a elegância do formalismo adotado aqui, como também a estabilidade proporcionada ao algoritmo desenvolvido nesta dissertação.

Visando uma avaliação do desempenho da cobertura de invisibilidade implementada neste trabalho, os resultados investigados pelo formalismo aqui desenvolvido foram comparados com os obtidos na literatura, tanto teóricos quanto experimentais [18]. Do ponto de vista qualitativo, os resultados estão em perfeita concordância.

Após a análise da cobertura de invisibilidade eletromagnética, foi investigada a estrutura capaz de proporcionar uma rotação nas frentes de ondas planas por um ângulo pré-estabelecido. Foi também investigado o quão sensível esta estrutura pode ser quanto a variações dos parâmetros de projeto (nesse caso, para a freqüência de operação), tendo os resultados sido apresentados em termos da seção de espalhamento 
da estrutura (SCS) normalizada pela freqüência de operação desse dispositivo. Como eram esperados, pequenos valores de SCS foram obtidos em freqüências próximas à freqüência na qual o rotacionador de campo foi projetado.

De modo geral, o formalismo 2D-FDTD implementado nesta dissertação mostrouse eficiente na investigação de estruturas capazes de proporcionar a manipulação da radiação eletromagnética, tendo os parâmetros eletromagnéticos desses dispositivos sido modelados por Drude. Esse formalismo revelou-se estável e preciso, caracterizando-o como uma boa contribuição na análise do funcionamento dessas estruturas.

Como proposta para trabalhos futuros, cita-se aplicar o formalismo aqui desenvolvido em outras estruturas, tais como hiperlentes e concentradores de campo, bem como, investigar a resposta eletromagnéticas de estruturas com outras formas geométricas. Será possível também, a realização de uma análise detalhada da cobertura de invisibilidade eletromagnética, tendo em vista que o problema de instabilidade numérica foi solucionado. 


\section{Referências Bibliográficas}

[1] R. W. Ziolkowski e N. Engheta, "Introduction, History, and Selected Topics in Fundamental Theories of Metamaterials" em N. Engheta e R. W. Ziolkowski, Metamaterials: Physics and Engineering Explorations, New York: Wiley-IEEE Press, 2006.

[2] V. G. Veselago, "Electrodynamics of media with simultaneously negative electric permittivity and magnetic permeability", em: S. Zouhdi, A. Sihvola, M. Arsalame, Advantaces in Electromagnetics of Complex Media and Metamaterials, vol. 89, Dordrech: Kluwer Academic Publishers, 2003.

[3] D. R. Smith, W. J. Padilla, D. C. Vier, S. C. Nemat-Nasser e S. Schultz, “Composite medium with simultaneous negative permeability and permittivity", Phys. Rev. Lett., $84,2000$.

[4] W. Rotman, "Plasma simulation by artificial dielectrics and parallel-plate media", IRE Trans. Antennas Propag, vol. AP-10, no. 82, 1962.

[5] J. B. Pendry, A. J. Holden, W. J. Stewart e I. Youngs, "Extremely low frequency plasmons in metallic mesostructures", Phys. Rev. Lett., 25, 1996.

[6] J. B. Pendry, A. J. Holden, D. J. Robbins e W. J. Stewart, "Magnetism from conductors and enhanced nonlinear phenomena", IEEE Trans. Microwave Theory Tech., 11, 1999.

[7] R. A. Shelby, D. R. Smith, S. C. Nemat-Nasser e S. Schultz, "Experimental verification of a negative index of refraction", Science, vol. 292, pp. 77-79, 2001.

[8] N. Jain, "EM design and applications of metamaterials", relatório do laboratório aeroespacial do instituto de tecnologia e ciência de Pilani, Índia, 2005. 
[9] http://apps.isiknowledge.com/

[10] A. Sihvola, "Electromagnetic emergence in Metamaterials", em: S. Zouhdi, A. Sihvola, M. Arsalame, Advantaces in Electromagnetics of Complex Media and Metamaterials, vol. 89, Dordrech, Kluwer Academic Publishers, 2003.

[11] A. Sihvola, "Metamaterials in electromagnetics", Metamaterials, vol. 1, pp. 2-7, 2007.

[12] http://www.metamorphose-vi.org/

[13] http://www.darpa.mil/

[14] M. Bayindir, B. Temelkuran e E. Ozbay, "Propagation of photons by hopping: A waveguiding mechanism through localized coupled cavities in three-dimensional photonic crystals," Phys. Rev. B 61, 2000.

[15] G. V. Eleftheriades e K. G. Balmain, Negative-Refraction Metamaterials, New Jersey: Wiley-IEEE Press, 2005.

[16] A. Lai, C. Caloz e T. Itoh, "Composite left-/Right-handed transmission line metamaterials," IEEE magazine, 2004.

[17] N. I. Landy, S. Sajuyigbe, J. J. Mock, D. R. Smith e W. J. Padilla, "Perfect Metamaterial Absorber", Phys. Rev. Letters, 100, Maio 2008.

[18] D. Schurig, J. J. Mock, B. J Justice, S. A. Cummer, J. B. Pendry, A. F. Starr and D. R. Smith, "Metamaterial Electromagnetic Cloak at Microwave Frequencies", Science, vol. 314, no. 5801, pp. 977-980, November 2006.

[19] J. B. Pendry, D. Schurig and D. R. Smith, "Controlling electromagnetic fields", Science, vol. 312, no. 5781, pp. 1780-1782, June 2006. 
[20] D. Schurig, J. B. Pendry and D. R. Smith, "Calculation of material properties and ray tracing in transformation media", Opt. Express, vol. 14, no. 21, pp. 97949804, October 2006.

[21] S. A. Cummer, B.-I. Popa, D. Schurig, and D. R. Smith, "Full-wave simulations of electromagnetic cloaking structures," Phys. Review E, vol. 74, July 2006.

[22] W. Cai, U. K. Chettiar, A. V. Kildishev and V. M. Shalaev, "Optical cloaking with metamaterials," Nature Photonics, vol. 1, pp. 224-228, April 2007.

[23] M. Tsang e D. Psaltis, "Magnifying perfect lens and superlens design by coordinate transformation," arXiv:0708.0262v3 (unpublished).

[24] A.V. Kildishev e E.E. Narimanov, "Impedance-matched hyperlens," Opt. Letters, vol. 32, pp. 3432-3434, Nov. 2007.

[25] M. Rahm D. Schurig D.A. Roberts S.A. Cummer D.R. Smith e J.B. Pendry, "Design of electromagnetic cloaks and concentrators using form-invariant coordinate transformations of Maxwell's equations," Phot. Nanostruc. Fund. and Appl., vol. 6, pp. 87-95, Aug. 2007.

[26] Y. Luo, H. Chen, J. Zhang, L. Ran e J. A. Kong, "Design and analytical fullwave validation of the invisibility cloaks, concentrators, and field rotators created with a general class of transformations,” Phys. Rev. Appl., vol. 77, pp. 125127/1125127/8, Mar. 2008.

[27] A.D. Yaghjian e S. Maci, "Alternative Derivation of Electromagnetic Cloaks and Concentrators,” arXiv:0710.2933, 2007 (unpublished).

[28] H. Chen, and C.T. Chan, "Transformation media that rotate electromagnetic fields,” Appl. Phys. Letters, vol. 90, pp. 241105/1-241105/3, Jun. 2007.

[29] U. Leonhardt, "Optimal conformal mapping", Science, vol. 312, no. 5781, pp. 1777-1780, Jun. 2006. 
[30] U. Leonhardt and T. G. Philbin, "General relativity in electrical engineering", New Journal of Physics, vol. 8, October 2006.

[31] A. Greenleaf, Y. Kurylev, M. Lassas and G. Uhlmann, "Full-Wave Invisibility of Active Devices at All Frequencies," Comm. Math. Physics, vol. 275, no. 3, pp. 749-789, August 2007.

[32] C. F. Bohren e D. R. Huffman, Absorption and Scattering of Light by Small Particles, New York: Wiley, 1983.

[33] E. J. Post, Formal Structure of Electromagnetics general covariance and electromagnetics, New York: Dover, 1997.

[34] J. I. Cisnero, Ondas Eletromagnéticas Fundamentos e Aplicações, Campinas, Editora da Unicamp, 2001.

[35] C. Kittel, Introduction to Solid State Physics, United States: John Wiley \& Sons, 1971.

[36] M. N. O. Sadiku, Numerical Techniques in Electromagnetics, United States of America: CRC Press, 2001.

[37] A. Taflove e S. C. Hagness, Computational Electrodynamics The FiniteDifference Time Domain Method, Norwood: Artech House, 2005.

[38] K. Kunz e R. J. Luebbers, Finite Difference Time Domain Method for Electromagnetics, Boca Raton, CRC Press LLC, 1993.

[39] K. S. Yee, "Numerical Solutions of Initial Boundary Value Problems Involving Maxwell's Equations in Isotropic Media”, IEEE Transactions on Antennas and Propagation, vol. 14, May 1966. 
[40] J. P. Berenger, "A Perfectly Matched Layer for the Absorptions of Electromagnetics Waves”, Journal of Computational Physics, vol. 114, 1994.

[41] J. A. S. Macêdo, M. A. Romero e B. H. V. Borges, "Improved FDTD formalism for electromagnetic cloaking applications", 13th Biennial IEEE Conference on Electromagnetic Field Computation - CEFC2008, Grécia, 2008.

[42] R.W. Ziolkowski e E. Heyman, "Wave propagation in media having negative permittivity and permeability", Phys Rev E, vol. 64, 2001.

[43] D. Correia e J. M. Jin, “3D-FDTD-PML Analysis of left-handed metamaterials”, Microwave and Optical Tech. Letters, vol. 3, 2004. 


\section{APÊNDICE A - Cálculo dos parâmetros eletromagnéticos da cobertura de invisibilidade}

Como definido na Fig. A1, a região onde os campos eletromagnéticos devem ser manipulados apresenta geometria circular. Sendo assim, considere inicialmente a região definida por $0<r<b$, Fig. A1(a). Para que o efeito de cobertura de invisibilidade seja possível, é necessário que o espalhador esteja colocado em uma região onde o campo eletromagnético não pode penetrar (sem ser perturbado). Isso pode ser obtido via uma transformação linear que expande a origem do sistema criando uma região que, para todos os efeitos, não existe para as equações de Maxwell. Se essa origem expandida apresenta um raio $a$, logo é possível obter um sistema transformado cuja geometria é ilustrada na Fig. A1(b).

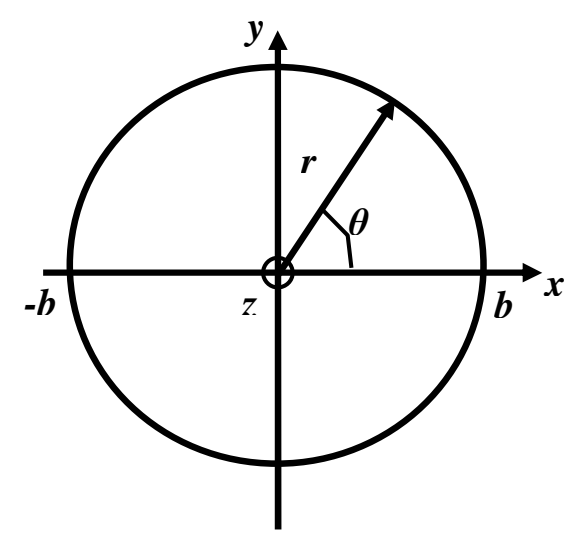

(a)

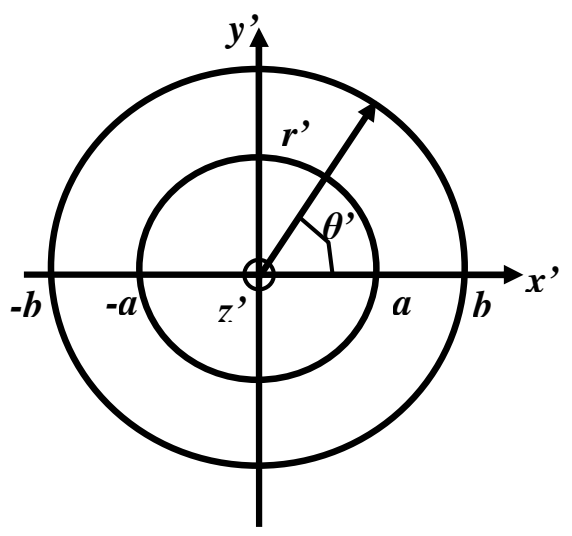

(b)

Fig. A1 - Representação da estrutura analisada no sistema cartesiano. (a) círculo delimitando a fronteira da região a ser transformada, (b) sistema transformado apresentando a origem expandida de raio $a$. 
A região onde os campos eletromagnéticos deverão ser adequadamente manipulados é dada pelo cilindro ilustrado na Fig. A.1(b). A partir desta figura, as seguintes relações podem ser obtidas:

$$
\begin{gathered}
y=r \sin \theta, \\
x=r \cos \theta, \\
r=\sqrt{x^{2}+y^{2}}, \\
z=z, \\
r=0 \Leftrightarrow r^{\prime}=a, \\
r=b \Leftrightarrow r^{\prime}=b,
\end{gathered}
$$

ou seja:

$$
\begin{gathered}
r^{\prime}=\frac{b-a}{b} r+a, \\
\theta^{\prime}=\theta, \\
z^{\prime}=z .
\end{gathered}
$$

A representação da estrutura analisada no sistema cartesiano é:

$$
\begin{gathered}
x^{\prime}=r^{\prime} \cos \theta^{\prime}, \\
y^{\prime}=r^{\prime} \sin \theta^{\prime}, \\
z=z^{\prime} .
\end{gathered}
$$


Substituindo (A.1)-(A.3) em (A.4)-(A.6) resulta em:

$$
\begin{gathered}
x^{\prime}=\left[\frac{b-a}{b} r+a\right] \cos \theta^{\prime}, \\
y^{\prime}=\left[\frac{b-a}{b} r+a\right] \sin \theta^{\prime}, \\
z^{\prime}=z .
\end{gathered}
$$

sendo:

$$
\begin{gathered}
r=\sqrt{x^{2}+y^{2}}, \\
\cos \theta=\cos \theta^{\prime}=\frac{x}{\sqrt{x^{2}+y^{2}}},
\end{gathered}
$$

e

$$
\sin \theta=\sin \theta^{\prime}=\frac{y}{\sqrt{x^{2}+y^{2}}}
$$

È obtido as equações que relacionam o sistema original com o sistema transformado:

$$
\begin{gathered}
x^{\prime}=\left[\frac{b-a}{b}\left(\sqrt{x^{2}+y^{2}}\right)+a\right] \frac{x}{\sqrt{x^{2}+y^{2}}}, \\
x^{\prime}=\frac{b-a}{b} x+\frac{a x}{r}, \\
y^{\prime}=\left[\frac{b-a}{b}\left(\sqrt{x^{2}+y^{2}}\right)+a\right] \frac{y}{\sqrt{x^{2}+y^{2}}}, \\
y^{\prime}=\frac{b-a}{b} y+\frac{a y}{r}, \\
z=z^{\prime} .
\end{gathered}
$$


Para que o efeito de invisibilidade ou o de rotação de campo seja obtido, é necessário que os parâmetros do meio sejam devidamente alterados, sem contudo alterar suas propriedades iniciais. Isso pode ser realizado alterando a permissividade e a permeabilidade por meio de uma lei linear e homogênea de transformação [1], definida por:

$$
\begin{aligned}
& \varepsilon_{r}^{i^{\prime} j^{\prime}}=\left|\operatorname{det}\left(\Lambda_{\alpha}^{\alpha^{\prime}}\right)\right|^{-1} \Lambda_{i}^{i^{\prime}} \Lambda_{j}^{j^{\prime}} \varepsilon_{r}^{i j}, \\
& \mu_{r}^{i^{\prime} j^{\prime}}=\left|\operatorname{det}\left(\Lambda_{\alpha}^{\alpha^{\prime}}\right)\right|^{-1} \Lambda_{i}^{i^{\prime}} \Lambda_{j}^{j^{\prime}} \mu_{r}^{i j} .
\end{aligned}
$$

onde:

$\Lambda_{\alpha}^{\alpha^{\prime}}$ é a matriz jacobiana [1] que relaciona o espaço original com o transformado, sendo definida por:

$$
\Lambda_{\alpha}^{\alpha^{\prime}}=\left[\begin{array}{lll}
\Lambda_{1}^{1^{\prime}} & \Lambda_{2}^{1^{\prime}} & \Lambda_{3}^{1^{\prime}} \\
\Lambda_{1}^{2^{\prime}} & \Lambda_{2}^{2^{\prime}} & \Lambda_{3}^{2^{\prime}} \\
\Lambda_{1}^{3^{\prime}} & \Lambda_{2}^{3^{\prime}} & \Lambda_{3}^{3^{\prime}}
\end{array}\right]=\left[\begin{array}{lll}
\frac{\partial x^{\prime}}{\partial x} & \frac{\partial x^{\prime}}{\partial y} & \frac{\partial x^{\prime}}{\partial z} \\
\frac{\partial y^{\prime}}{\partial x} & \frac{\partial y^{\prime}}{\partial y} & \frac{\partial y^{\prime}}{\partial z} \\
\frac{\partial z^{\prime}}{\partial x} & \frac{\partial z^{\prime}}{\partial y} & \frac{\partial z^{\prime}}{\partial z}
\end{array}\right]
$$

$\Lambda_{i, j}^{i^{\prime}, j^{\prime}}$ representa os seus elementos $(i$ ou $j=1,2,3), \varepsilon_{r}^{i j}$ e $\mu_{r}^{i j}$ são os elementos do tensor permissividade elétrica relativa e permeabilidade magnética relativa, respectivamente, do material não transformado. Os cálculos das derivadas são mostrados em detalhes a seguir: 


$$
\begin{aligned}
\frac{\partial x^{\prime}}{\partial x}= & \frac{\partial\left(\frac{b-a}{b} x\right)}{\partial x}+\frac{\partial\left(\frac{a x}{r}\right)}{\partial x} \\
= & \frac{b-a}{b}+\frac{a r-a x \cos \theta}{r^{2}}= \\
= & \frac{b-a}{b}+\frac{a}{r}-\frac{a x \cos \theta}{r^{2}}= \\
= & \frac{b-a}{b}+\frac{a}{r}-\frac{a}{r} \cos ^{2} \theta \\
\frac{\partial x^{\prime}}{\partial x}= & \frac{\partial\left(\frac{b-a}{b} x\right)}{\partial y}+\frac{\partial\left(\frac{a x}{r}\right)}{\partial y} \\
= & \frac{a y \cos \theta}{r^{2}}, \\
= & \frac{a x \sin \theta}{r^{2}}, \\
& \frac{\partial y^{\prime}}{\partial x}=\frac{a \cos \theta \sin \theta}{r}+\frac{a}{r} \sin ^{2} \theta \\
& \frac{\partial x^{\prime}}{\partial y}=\frac{a \cos \theta \sin \theta}{r}
\end{aligned}
$$




$$
\begin{gathered}
\frac{\partial y^{\prime}}{\partial y}=\frac{\partial\left(\frac{b-a}{b} y\right)}{\partial y}+\frac{\partial\left(\frac{a y}{r}\right)}{\partial y} \\
=\frac{b-a}{b}+\frac{a r-a y \sin \theta}{r^{2}}= \\
=\frac{b-a}{b}+\frac{a}{r}-\frac{a y \sin \theta}{r^{2}}= \\
=\frac{b-a}{b}+\frac{a}{r}-\frac{a}{r} \sin ^{2} \theta \\
\frac{\partial y^{\prime}}{\partial y}=\frac{b-a}{b}+\frac{a}{r} \cos ^{2} \theta \\
\frac{\partial z^{\prime}}{\partial y}=0 \\
\frac{\partial x^{\prime}}{\partial z}=0 \\
\frac{\partial z^{\prime}}{\partial z}=1 .
\end{gathered}
$$

Deste modo, a matriz jacobiana é reescrita como:

$$
\Lambda_{\alpha}^{\alpha^{\prime}}=\left[\begin{array}{ccc}
\frac{b-a}{b}+\frac{a}{r} \sin ^{2} \theta & \frac{a \cos \theta \sin \theta}{r} & 0 \\
\frac{a \cos \theta \sin \theta}{r} & \frac{b-a}{b}+\frac{a}{r} \cos ^{2} \theta & 0 \\
0 & 0 & 1
\end{array}\right] .
$$


68

O determinante desta matriz é calculado a seguir:

$$
\operatorname{det}\left(\Lambda_{\alpha}^{\alpha^{\prime}}\right)=\left\{\begin{array}{l}
{\left[\left(\frac{b-a}{b}+\frac{a}{r} \sin ^{2} \theta\right) \cdot\left(\frac{b-a}{b}+\frac{a}{r} \cos ^{2} \theta\right) \cdot 1\right]-} \\
{\left[\left(\frac{a \cos \theta \sin \theta}{r}\right) \cdot\left(\frac{a \cos \theta \sin \theta}{r}\right) \cdot 1\right]}
\end{array}\right\} .
$$

Sendo $r=\frac{\left(r^{\prime}-a\right) b}{b-a}$, tem-se:

$$
\begin{gathered}
\operatorname{det}\left(\Lambda_{\alpha}^{\alpha^{\prime}}\right)=\left\{\left[\left(\frac{b-a}{b}+\left(\frac{b-a}{b}\right)\left(\frac{a}{r^{\prime}-a}\right) \sin ^{2} \theta\right) \cdot\left(\frac{b-a}{b}+\left(\frac{b-a}{b}\right)\left(\frac{a}{r^{\prime}-a}\right) \cos ^{2} \theta\right) \cdot 1\right]-\right. \\
\left.\left[\left(\frac{b-a}{b}\right)\left(\frac{a}{r^{\prime}-a}\right) \cos \theta \sin \theta \cdot\left(\frac{b-a}{b}\right)\left(\frac{a}{r^{\prime}-a}\right) \cos \theta \sin \theta \cdot 1\right]\right\}, \\
\operatorname{det}\left(\Lambda_{\alpha}^{\alpha^{\prime}}\right)=\left\{\left[\left(\frac{b-a}{b}\right) \cdot\left(1+\left(\frac{a}{r^{\prime}-a}\right) \sin ^{2} \theta\right) \cdot\left(\frac{b-a}{b}\right) \cdot\left(1+\left(\frac{a}{r^{\prime}-a}\right) \cos ^{2} \theta\right) \cdot 1\right]-\right. \\
\left.\left[\left(\frac{b-a}{b}\right)\left(\frac{a}{r^{\prime}-a}\right) \cos \theta \sin \theta \cdot\left(\frac{b-a}{b}\right)\left(\frac{a}{r^{\prime}-a}\right) \cos \theta \sin \theta \cdot 1\right]\right\}, \\
\operatorname{det}\left(\Lambda_{\alpha}^{\alpha^{\prime}}\right)=\left\{\left(\frac{b-a}{b}\right)^{2} \cdot\left[\left(\frac{r^{\prime}-a+a \sin { }^{2} \theta}{r^{\prime}-a}\right) \cdot\left(\frac{r^{\prime}-a+a \cos { }^{2} \theta}{r^{\prime}-a}\right)\right]-\right. \\
\left.\left(\frac{b-a}{b}\right)^{2} \cdot\left[\left(\frac{a}{r^{\prime}-a}\right) \cos \theta \sin \theta \cdot\left(\frac{a}{r^{\prime}-a}\right) \cos \theta \sin \theta\right]\right\}, \\
\operatorname{det}\left(\Lambda_{\alpha}^{\alpha^{\prime}}\right)=\left\{\left(\frac{b-a}{b}\right)^{2} \cdot\left(\frac{1}{r^{\prime}-a}\right)^{2} \cdot\left[\left(r^{\prime}-a \cos ^{2} \theta\right) \cdot\left(r^{\prime}-a \sin { }^{2} \theta\right)\right]-\right. \\
\left.\left(\frac{b-a}{b}\right)^{2} \cdot\left(\frac{1}{r^{\prime}-a}\right)^{2} \cdot\left[a^{2} \cos ^{2} \theta \sin ^{2} \theta\right]\right\},
\end{gathered}
$$




$$
\begin{aligned}
& \operatorname{det}\left(\Lambda_{\alpha}^{\alpha^{\prime}}\right)=\left\{\left(\frac{b-a}{b}\right)^{2} \cdot\left(\frac{1}{r^{\prime}-a}\right)^{2} \cdot\left[\left(r^{\prime}-a \cos ^{2} \theta\right) \cdot\left(r^{\prime}-a \sin ^{2} \theta\right)\right]-\right. \\
&\left.\left(\frac{b-a}{b}\right)^{2} \cdot\left(\frac{1}{r^{\prime}-a}\right)^{2} \cdot\left[a^{2} \cos ^{2} \theta \sin ^{2} \theta\right]\right\}, \\
& \operatorname{det}\left(\Lambda_{\alpha}^{\alpha^{\prime}}\right)=\left\{\left(\frac{b-a}{b}\right)^{2} \cdot\left(\frac{1}{r^{\prime}-a}\right)^{2} \cdot\left[\left(r^{\prime 2}-r^{\prime} a \cos ^{2} \theta-r^{\prime} a \sin ^{2} \theta+a^{2} \cos ^{2} \theta \sin ^{2} \theta\right)\right]-\right. \\
&\left.\left(\frac{b-a}{b}\right)^{2} \cdot\left(\frac{1}{r^{\prime}-a}\right)^{2} \cdot\left[a^{2} \cos ^{2} \theta \sin ^{2} \theta\right]\right\}, \\
& \operatorname{det}\left(\Lambda_{\alpha}^{\alpha^{\prime}}\right)=\left(\frac{b-a}{b}\right)^{2} \cdot\left(\frac{1}{r^{\prime}-a}\right)^{2} \cdot\left(r^{\prime 2}-r^{\prime} a \cos ^{2} \theta-r^{\prime} a \sin ^{2} \theta\right) \\
&=\left(\frac{b-a}{b}\right)^{2} \cdot\left(\frac{1}{r^{\prime}-a}\right)^{2}\left(r^{\prime 2}-r^{\prime} a\right) \cdot\left(\cos ^{2} \theta+\sin ^{2} \theta\right) .
\end{aligned}
$$

Resultando em:

$$
\operatorname{det}\left(\Lambda_{\alpha}^{\alpha^{\prime}}\right)=\left(\frac{b-a}{b}\right)^{2} \cdot\left(\frac{r^{\prime}}{r^{\prime}-a}\right)
$$

Logo:

$$
\left|\operatorname{det}\left(\Lambda_{\alpha}^{\alpha^{\prime}}\right)\right|^{-1}=\left(\frac{b}{b-a}\right)^{2} \cdot\left(\frac{r^{\prime}-a}{r^{\prime}}\right)
$$

Substituindo (A.12)-(A.21) em (A.10) e (A.11), calculam-se os nove elementos dos tensores dos parâmetros eletromagnéticos, mostrado a seguir. Considerando que o material original é o espaço-livre, tem-se:

$$
\varepsilon_{r}^{i^{\prime} j^{\prime}}=\mu_{r}^{i^{\prime} j^{\prime}}=\left|\operatorname{det}\left(A_{i}^{i^{\prime}}\right)\right|^{-1} A_{i}^{i^{\prime}} A_{j}^{j^{\prime}} .
$$


Os elementos dos tensores são calculados a seguir:

$$
\begin{aligned}
\mathcal{E}_{r}^{1^{\prime I^{\prime}}}=\mu_{r}^{1^{\prime \prime \prime}}=\left|\operatorname{det}\left(\Lambda_{\alpha}^{\alpha^{\prime}}\right)\right|^{-1}\left(\Lambda_{1}^{1^{\prime}} \Lambda_{1}^{1^{\prime}}+\Lambda_{2}^{1^{\prime}} \Lambda_{2}^{1^{\prime}}+\Lambda_{3}^{1^{\prime}} \Lambda_{3}^{1^{\prime}}\right) \cdot 1 & {\left[\left(\frac{b}{b-a}\right)^{2} \cdot\left(\frac{r^{\prime}-a}{r^{\prime}}\right)\right] \cdot\left[\left(\frac{b-a}{b}\right)^{2} \cdot\left(\frac{1}{r^{\prime}-a}\right)^{2} \cdot\left(r^{\prime}-a \cos ^{2} \theta\right)^{2}+\right.} \\
& \left.\left(\frac{b-a}{b}\right)^{2} \cdot\left(\frac{1}{r^{\prime}-a}\right)^{2} \cdot(a \cos \theta \sin \theta)^{2}\right] \\
\mathcal{E}_{r}^{1^{\prime \prime} 1^{\prime}}=\mu_{r}^{1^{\prime \prime} 1^{\prime}}= & \frac{1}{r^{\prime}}\left[\frac{\left(r^{\prime}-a \cos ^{2} \theta\right)^{2}}{r^{\prime}-a}+\frac{(a \cos \theta \sin \theta)^{2}}{r^{\prime}-a}\right] \\
= & \frac{r^{\prime 2}-2 r^{\prime} a \cos ^{2} \theta+a^{2} \cos ^{4} \theta+a^{2} \cos ^{2} \theta \sin ^{2} \theta}{r^{\prime}\left(r^{\prime}-a\right)} .
\end{aligned}
$$

Sabendo que

$$
r^{\prime 2}=r^{\prime 2} \cos ^{2} \theta+r^{\prime 2} \sin ^{2} \theta
$$

obtém-se:

$$
\begin{aligned}
\mathcal{E}_{r}^{1^{\prime \prime}}=\mu_{r}^{\prime 1^{\prime}} & =\frac{r^{\prime 2} \cos ^{2} \theta+r^{\prime 2} \sin ^{2} \theta-2 r^{\prime} a \cos ^{2} \theta+a^{2} \cos ^{2} \theta\left(\cos ^{2} \theta+\sin ^{2} \theta\right)}{r^{\prime}\left(r^{\prime}-a\right)} \\
& =\frac{r^{\prime 2} \cos ^{2} \theta-2 r^{\prime} a \cos ^{2} \theta+a^{2} \cos ^{2} \theta+r^{\prime 2} \sin ^{2} \theta}{r^{\prime}\left(r^{\prime}-a\right)} \\
& =\frac{\left(r^{\prime 2}-2 r^{\prime} a+a^{2}\right) \cos ^{2} \theta+r^{\prime 2} \sin ^{2} \theta}{r^{\prime}\left(r^{\prime}-a\right)}
\end{aligned}
$$

o que resulta em:

$$
\varepsilon_{r}^{1^{\prime} 1^{\prime}}=\mu_{r}^{11^{\prime}}=\frac{r^{\prime}-a}{r^{\prime}} \cos ^{2} \theta+\frac{r^{\prime}}{r^{\prime}-a} \sin ^{2} \theta
$$




$$
\begin{aligned}
& \varepsilon_{r}^{2^{\prime 2} 2^{\prime}}=\mu_{r}^{2^{\prime 2} 2^{\prime}}=\left|\operatorname{det}\left(\Lambda_{\alpha}^{\alpha^{\prime}}\right)\right|^{-1}\left(\Lambda_{1}^{2^{\prime}} \Lambda_{1}^{2^{\prime}}+\Lambda_{2}^{2^{\prime}} \Lambda_{2}^{2^{\prime}}+\Lambda_{3}^{2^{\prime}} \Lambda_{3}^{2^{\prime}}\right) 1 \\
& =\left[\left(\frac{b}{b-a}\right)^{2} \cdot\left(\frac{r^{\prime}-a}{r^{\prime}}\right)\right] \cdot\left[\left(\frac{b-a}{b}\right)^{2} \cdot\left(\frac{1}{r^{\prime}-a}\right)^{2} \cdot(a \cos \theta \sin \theta)^{2}+\right. \\
& \left.\left(\frac{b-a}{b}\right)^{2} \cdot\left(\frac{1}{r^{\prime}-a}\right)^{2} \cdot\left(r^{\prime}-a \sin ^{2} \theta\right)^{2}\right], \\
& \varepsilon_{r}^{2^{\prime 2} 2^{\prime}}=\mu_{r}^{2^{\prime 2} 2^{\prime}}=\frac{1}{r^{\prime}}\left[\frac{\left(r^{\prime}-a \sin ^{2} \theta\right)^{2}}{r^{\prime}-a}+\frac{(a \cos \theta \sin \theta)^{2}}{r^{\prime}-a}\right] \\
& =\frac{r^{\prime 2}-2 r^{\prime} a \sin ^{2} \theta+a^{2} \sin ^{4} \theta+a^{2} \cos ^{2} \theta \sin ^{2} \theta}{r^{\prime}\left(r^{\prime}-a\right)} .
\end{aligned}
$$

sendo:

$$
r^{\prime 2}=r^{\prime 2} \cos ^{2} \theta+r^{\prime 2} \sin ^{2} \theta .
$$

tem-se:

$$
\begin{aligned}
\mathcal{E}_{r}^{2^{\prime 2} 2^{\prime}}=\mu_{r}^{2^{\prime 2}} & =\frac{r^{\prime 2} \cos ^{2} \theta+r^{\prime 2} \sin ^{2} \theta-2 r^{\prime} a \sin ^{2} \theta+a^{2} \sin ^{2} \theta\left(\cos ^{2} \theta+\sin ^{2} \theta\right)}{r^{\prime}\left(r^{\prime}-a\right)} \\
& =\frac{r^{\prime 2} \sin ^{2} \theta-2 r^{\prime} a \sin ^{2} \theta+a^{2} \sin ^{2} \theta+r^{\prime 2} \cos ^{2} \theta}{r^{\prime}\left(r^{\prime}-a\right)} \\
& =\frac{\left(r^{\prime 2}-2 r^{\prime} a+a^{2}\right) \sin ^{2} \theta+r^{\prime 2} \cos ^{2} \theta}{r^{\prime}\left(r^{\prime}-a\right)}
\end{aligned}
$$

resultando em:

$$
\begin{gathered}
\varepsilon_{r}^{2^{\prime 2} 2^{\prime}}=\mu_{r}^{2^{\prime} 2^{\prime}}=\frac{r^{\prime}-a}{r^{\prime}} \sin ^{2} \theta+\frac{r^{\prime}}{r^{\prime}-a} \cos ^{2} \theta, \\
\varepsilon_{r}^{3^{3} 3^{\prime}}=\mu_{r}^{3^{\prime} 3^{\prime}}=\left|\operatorname{det}\left(\Lambda_{\alpha}^{\alpha^{\prime}}\right)\right|^{-1}\left(\Lambda_{1}^{3^{\prime}} \Lambda_{1}^{3^{\prime}}+\Lambda_{2}^{3^{\prime}} \Lambda_{2}^{3^{\prime}}+\Lambda_{3}^{3^{\prime}} \Lambda_{3}^{3^{\prime}}\right) \cdot 1 \\
\varepsilon_{r}^{3^{33^{\prime}}}=\mu_{r}^{3^{\prime} 3^{\prime}}=\left[\left(\frac{b}{b-a}\right)^{2} \cdot\left(\frac{r^{\prime}-a}{r^{\prime}}\right)\right],
\end{gathered}
$$




$$
\begin{aligned}
& \varepsilon_{r}^{1^{\prime 2^{\prime}}}=\mu_{r}^{1^{\prime 2} 2^{\prime}}=\varepsilon_{r}^{2^{\prime} 1^{\prime}}=\mu_{r}^{21^{\prime}}=\left|\operatorname{det}\left(\Lambda_{\alpha}^{\alpha^{\prime}}\right)\right|^{-1}\left(\Lambda_{1}^{1^{\prime}} \Lambda_{1}^{2^{\prime}}+\Lambda_{2}^{1^{\prime}} \Lambda_{2}^{2^{\prime}}+\Lambda_{3}^{1^{\prime}} \Lambda_{3}^{2^{\prime}}\right) \cdot 1 \\
& =\left[\left(\frac{b}{b-a}\right)^{2} \cdot\left(\frac{r^{\prime}-a}{r^{\prime}}\right)\right] \cdot\left(\frac{b-a}{b}\right)^{2} \cdot\left(\frac{1}{r^{\prime}-a}\right)^{2} \cdot\left[\left(r^{\prime}-a \cos ^{2} \theta\right) \cdot(a \cos \theta \sin \theta)+\right. \\
& =\frac{\left(\left(r^{\prime} a-a^{2} \cos ^{2} \theta\right)+\left(r^{\prime} a-a^{2} \sin ^{2} \theta\right)\right](\cos \theta \sin \theta)}{r^{\prime}\left(r^{\prime}-a\right)} .
\end{aligned}
$$

O que resulta em:

$$
\varepsilon_{r}^{1^{2^{\prime}}}=\mu_{r}^{1^{\prime \prime} 2^{\prime}}=\varepsilon_{r}^{2^{\prime} 1^{\prime}}=\mu_{r}^{2^{\prime} 1^{\prime}}=\frac{a^{2}-2 r^{\prime} a}{r^{\prime}\left(r^{\prime}-a\right)} \cos \theta \sin \theta
$$

\section{Referência}

[44] E. J. Post, Formal Structure of Electromagnetics General Covariance and Electromagnetics, New York: Dover, 1997. 


\section{APÊNDICE B - Fluxograma do algoritmo FDTD}

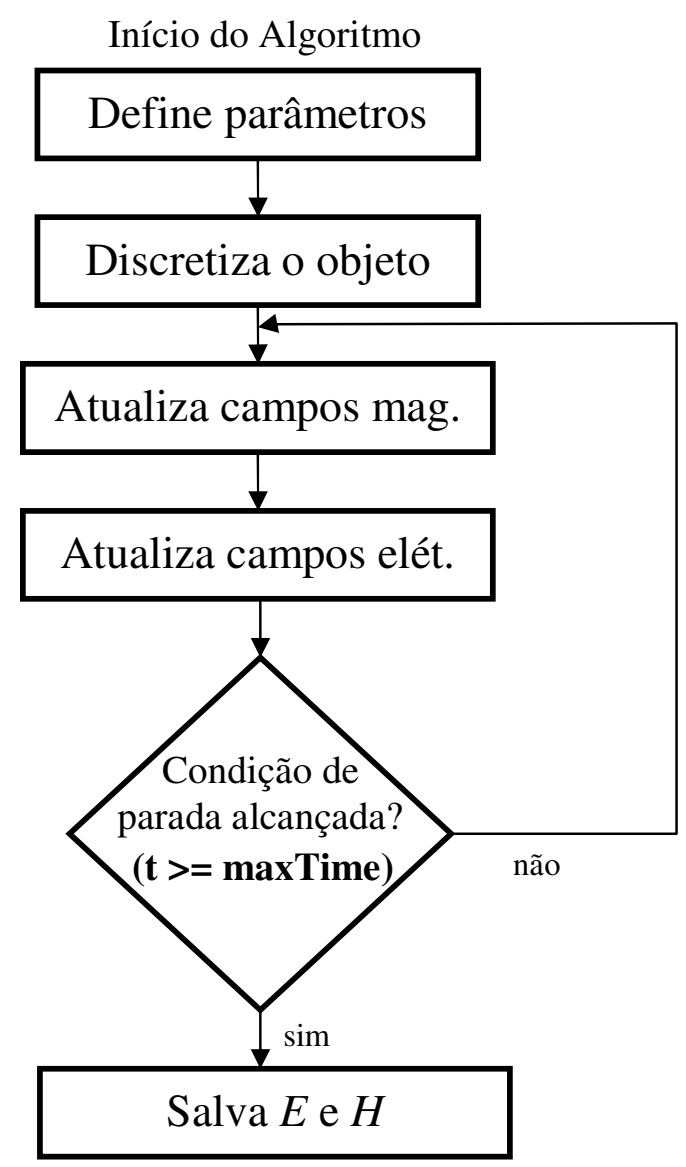

Fim do Algoritmo

Fig. B.1 - Fluxograma do algoritmo FDTD. 


\section{APÊNDICE C - Condições de contorno absorventes na borda do domínio computacional}

A condição de contorno adotada para o truncamento da janela computacional é a de paredes perfeitamente casadas (PML) proposta por Berenger [1]. O funcionamento correto desta condição de contorno requer que os perfis de condutividade elétrica $(\sigma)$ e magnética $\left(\sigma^{*}\right)$, inerentes a este formalismo, sejam precisamente calculados. Desta forma, um casamento perfeito de impedância de onda entre a camada absorvedora e o espaço-livre seja obtido sob qualquer ângulo de incidência pode ser obtido, eliminando reflexões indesejadas. O cálculo desses parâmetros é apresentado a seguir.

A Fig.C.1 ilustra o domínio computacional envolvido pela PML, vista sob dois planos diferentes, ou seja, plano $x z$ (Fig. C1(a)) e plano $x y$ (Fig. C1(b)),respectivamente. Nessa figura, é possível verificar um esquema ilustrativo da variação espacial da condutividade elétrica na direção $x$ (plano $x z$ ).

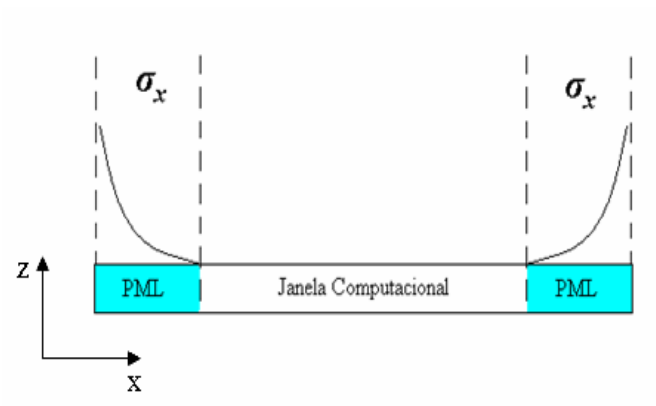

(a)

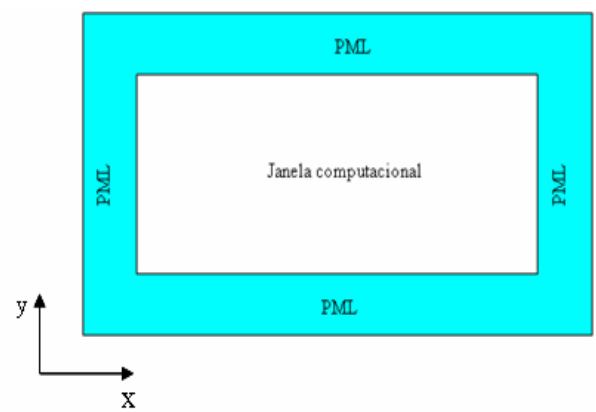

(b)

Fig.C.1 - Janela computacional terminada com PML. (a) estrutura vista no plano $x z$ e (b)no plano $x y$.

A formulação geral da PML é [1]: 


$$
\begin{gathered}
\varepsilon_{0} \frac{\partial E_{x}}{\partial t}=\frac{\partial H_{z}}{\partial y} \\
\varepsilon_{0} \frac{\partial E_{y}}{\partial t}=-\frac{\partial H_{z}}{\partial x} \\
\varepsilon_{0} \frac{\partial E_{z}}{\partial t}+\sigma_{x} E_{z}=\frac{\partial H_{y}}{\partial x} \\
\mathcal{E}_{0} \frac{\partial E_{z}}{\partial t}+\sigma_{y} E_{z}=-\frac{\partial H_{x}}{\partial y} \\
\mu_{0} \frac{\partial H_{z}}{\partial t}+\sigma_{y}^{*} H_{z}=\frac{\partial E_{x}}{\partial y} \\
\mu_{0} \frac{\partial H_{x}}{\partial t}=-\frac{\partial E_{z}}{\partial y} \\
\mu_{0} \frac{\partial H_{y}}{\partial t}=\frac{\partial E_{z}}{\partial x}
\end{gathered}
$$

Para um bom casamento de impedância entre a camada absorvedora e o domínio computacional do problema, a condição a seginte condição deve ser satisfeita [1]:

$$
\frac{\sigma}{\varepsilon_{0}}=\frac{\sigma^{*}}{\mu_{0}}
$$

Adicionalmente, adotou-se a expressão matemática da condutividade elétrica utilizada em [2], dada por:

$$
\sigma(\delta)=(\delta / d)^{m} \sigma_{\max }
$$


onde:

$\delta$ é distância que varia desde um valor inicial estabelecido na interface da PML com espaço-livre, até um valor máximo no final da PML, $d$ é a espessura da PML, $m$ representa o grau do polinômio dessa expressão e, $\sigma_{\max }$ é o máximo valor que a condutividade elétrica pode assumir.

Conforme descrito em [2], a escolha dos parâmetros $m$ e $\sigma_{\max }$ são importantes para se obter um bom desempenho da PML. Valores típicos de $m$ estão situados na faixa $2 \leq m \leq 4$ para simulações de FDTD [2]. Além disso, a precisão do método pode ser comprometida na medida em que $\sigma$ assume valores altos rapidamente. Diante deste problema, é necessário que a variação da condutividade elétrica não seja abrupta, e inicie com um dielétrico perfeito $(\sigma=0)$, passando por um dielétrico imperfeito $(\sigma / \omega \varepsilon<<1)$, até chegar a um bom condutor $(\sigma / \omega \varepsilon>>1)$. A condição de dielétrico perfeito e imperfeito pode ser alcançada através da razão $\delta / d$, conforme pode ser verificado em (C.2). Para atingir a condição de um bom condutor é necessária somente uma manipulação matemática com a condutividade elétrica máxima, e dessa forma concluir que $\sigma_{\max } \gg \omega \varepsilon_{0}$. O valor da condutividade máxima obedece a seguinte expressão:

$$
\sigma_{\max }=C \omega \varepsilon_{0}
$$

Em simulações que utilizam um número inferior a 10 camadas de PML, o valor da constante $C$ tem que ser calculado de forma que a condição de dielétrico imperfeito seja satisfeita $\left(\sigma_{\max } / \omega \varepsilon_{0}<<1\right)$. Logo, quando a razão

$$
\frac{\sigma_{\max }}{\omega \varepsilon_{0}} \cong 0,1
$$

for satisfeita, será considerado que o material é um dielétrico imperfeito, e quando

$$
\frac{\sigma_{\max }}{\omega \varepsilon_{0}} \cong 10
$$


será considerado que o material é um bom condutor. Deste modo, conclui-se que, o valor de $C$ deve ser um número igual ou superior a 10 para satisfazer tanto (C.4) quanto (C.5), quando o número de camadas de PML utilizadas na simulação for inferior a 10. Foi verificado que a faixa de variação obtida para $C$, em simulações que adotam de 8 a 10 camadas de PML está compreendido na faixa $10 \leq C \leq 15$.

\section{Referência}

[45] J. P. Berenger, "A Perfectly Matched Layer for the Absorptions of Electromagnetics Waves”, Journal of Computational Physics, vol. 114, 1994.

[46] A. Taflove e S. C. Hagness, Computational Electrodynamics The FiniteDifference Time Domain Method, Norwood: Artech House, 2005. 